\title{
AN INVESTIGATION INTO THE FREE VIBRATIONS OF CARBON NANOTUBES USING ANALYTICAL AND FINITE ELEMENT METHODS
}

\author{
by
}

Ishan Ali Khan,

B. Tech, Jawaharlal Nehru Technological University, Hyderabad (2011)

A thesis presented to Ryerson University in partial

fulfillment of the requirements for the degree of

Master of Applied Science in the Program of

Aerospace Engineering

Toronto, Ontario, Canada, 2016

CIshan Ali Khan 2016 


\section{Author's Declaration}

I hereby declare that I am the sole author of this thesis. This is the true copy of the thesis, including any required final revisions, as accepted by my examiners.

I authorize Ryerson University to lend this thesis to other institutions or individuals for the purpose of scholarly research.

I further authorize Ryerson University to reproduce this thesis by photocopying or by other means, in total or in part, at the request of other institutions or individuals for the purpose of scholarly research.

I understand that my thesis may be made electronically available to the public. 


\title{
AN INVESTIGATION INTO THE FREE VIBRATIONS OF \\ CARBON NANOTUBES USING ANALYTICAL AND FINITE ELEMENT METHODS
}

\author{
Ishan Ali Khan \\ Master of Applied Science, Aerospace Engineering, Ryerson University, Toronto (2016)
}

\begin{abstract}
Since their discovery, immense attention has been given to carbon nanotubes (CNTs), due to their exceptional thermal, electronic and mechanical properties and, therefore, the wide range of applications in which they are, or can be potentially, employed. Hence, it is important that all the properties of carbon nanotubes are studied extensively.
\end{abstract}

This thesis studies the vibrational frequencies of double-walled and triple-walled CNTs, with and without an elastic medium surrounding them, by using Finite Element Method (FEM) and Dynamic Stiffness Matrix (DSM) formulations, considering them as Euler-Bernoulli beams coupled with van der Waals interaction forces.

For FEM modelling, the linear eigenvalue problem is obtained using Galerkin weighted residual approach. The natural frequencies and mode shapes are derived from eigenvalues and eigenvectors, respectively. For DSM formulation of double-walled CNTs, a nonlinear eigenvalue problem is obtained by enforcing displacement and load end conditions to the exact solution of single equation achieved by combining the coupled governing equations. The natural frequencies are obtained using Wittrick-Williams algorithm. FEM formulation is also applied to both double and triple-walled CNTs modelled as nonlocal Euler-Bernoulli beam. The natural frequencies obtained for all the cases, are in agreement with the values provided in literature. 


\section{Acknowledgments}

I would like to extend my deepest gratitude to Prof. Seyed M. Hashemi for his continued support, guidance and patience throughout my research, and for his motivation and inspiration when I needed them.

I would also like to thank PhD candidates Supun Jayasinghe and Mir Tahmaseb Kashani, for enlightening discussions.

Finally, I would like to thank my family and friends, here and back home, for their continued support and faith in me. 


\section{Dedication}

I would like to dedicate this thesis to the memory of my father, to the inspiration of my mother, to the love and support of my family, and to the joyous laughter of my nephew, Usman. 


\section{Contents}

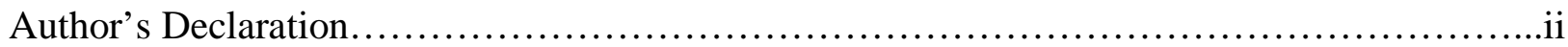

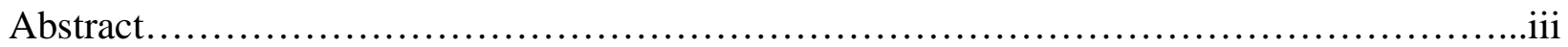

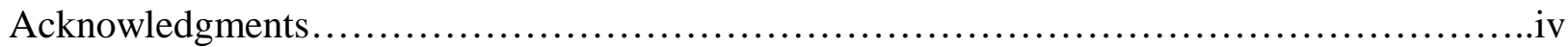

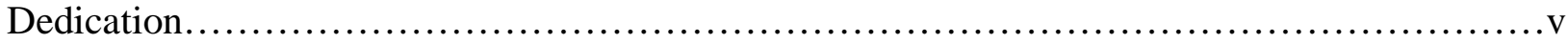

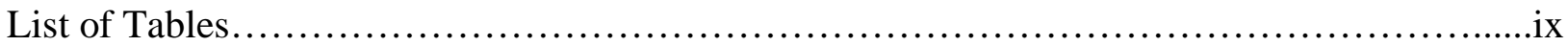

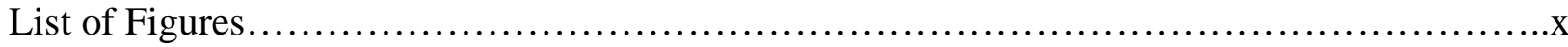

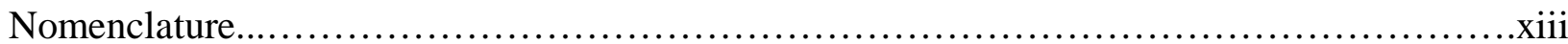

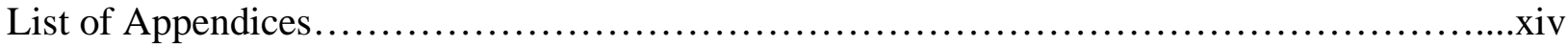

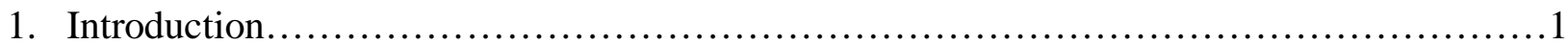

1.1. Outline of Finite Element Method...................................................

1.2. Outline of Dynamic Stiffness Method.................................................

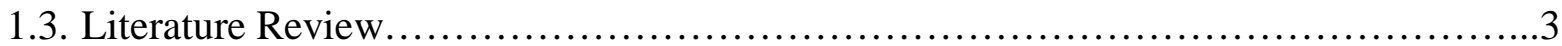

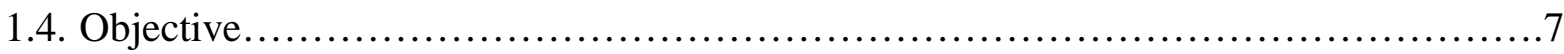

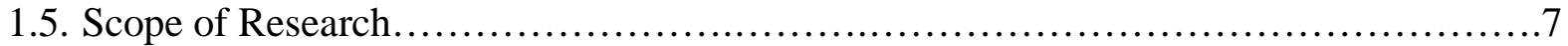

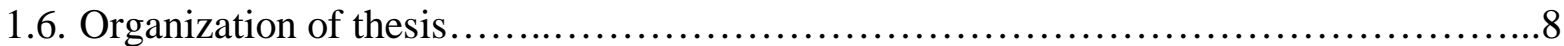

2. Carbon Nanotubes - A Brief Introduction................................................ 9

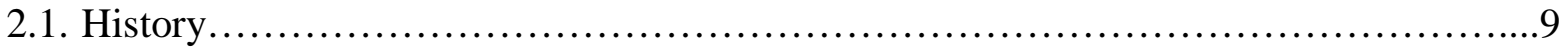

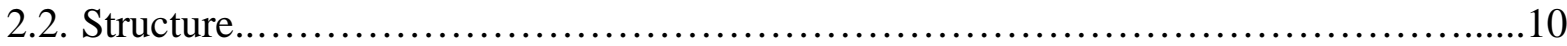

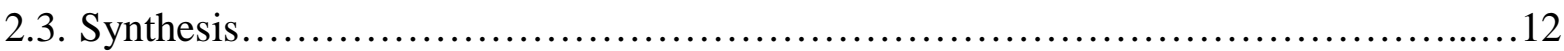

2.3.1. Arc Discharge Method.........................................................

2.3.2. Laser Ablation Method.........................................................12

2.3.3. Chemical Vapor Deposition...................................................13

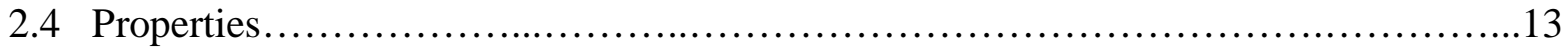

2.4.1 Mechanical Properties..........................................................13

2.4.2 Electronic Properties..........................................................

2.4.3 Thermal Properties........................................................... 15

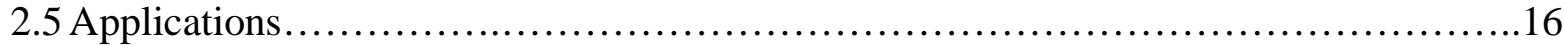

3. Finite Element Formulation - Double-walled Carbon Nanotubes............................18

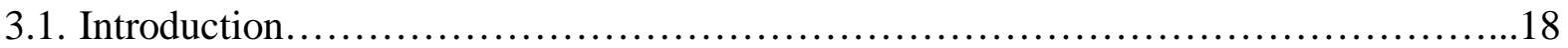




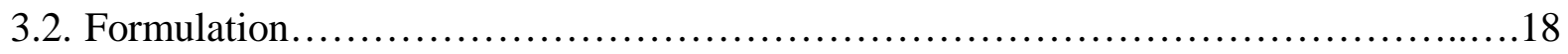

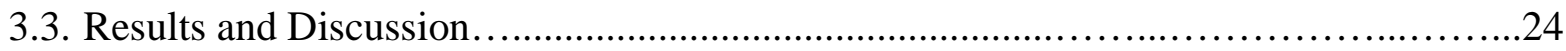

3.3.1. Double-walled Carbon Nanotubes modeled as local Euler-Bernoulli beam..........24

3.3.2. Double-walled Carbon Nanotubes, modeled as local Euler-Bernoulli beam with an elastic medium.........................................................

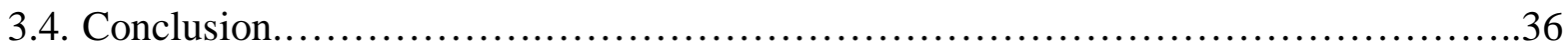

4. Dynamic Stiffness Matrix Formulation - Double-walled Carbon Nanotubes...................37

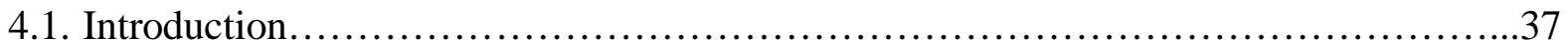

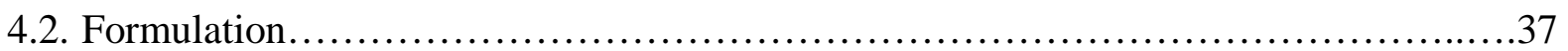

4.3. Wittrick-Williams Method....................................................44

4.4. Results and Discussion........................................................... 45

4.4.1. DWCNT modeled as local Euler-Bernoulli beam..............................45

4.4.2. DWCNT modeled as local Euler-Bernoulli beam embedded in elastic medium..46

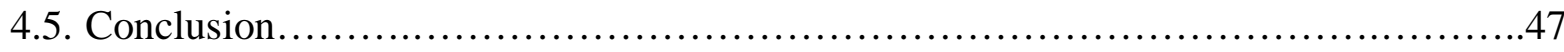

5. Finite Element Formulation - Triple-walled Carbon Nanotubes............................48

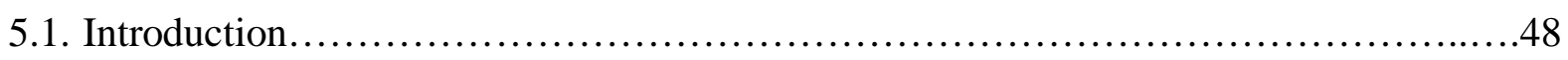

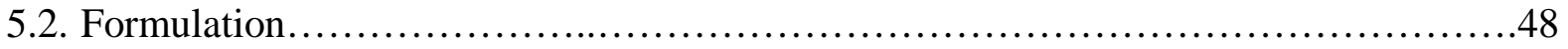

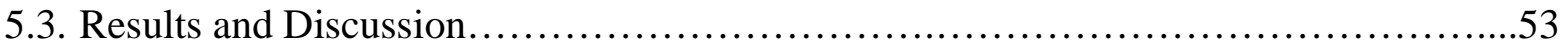

5.3.1. TWCNTs modeled as local Euler-Bernoulli beam............................54

5.3.2. TWCNTs modeled as local Euler-Bernoulli beam embedded in elastic medium.58

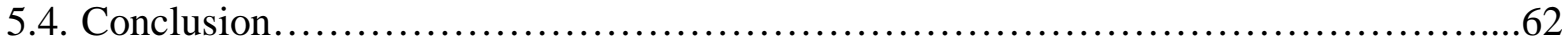

6. Nonlocal Finite Element Formulation..................................................63

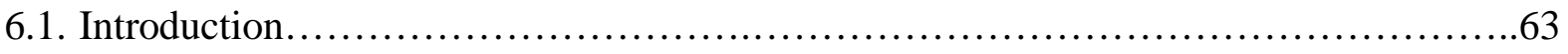

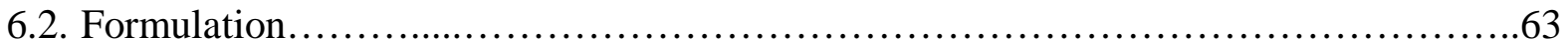

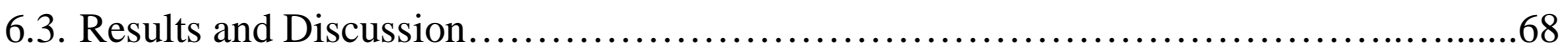

6.3.1. DWCNT modeled as nonlocal Euler-Bernoulli beam without elastic medium....68

6.3.2. DWCNT modeled as nonlocal Euler-Bernoulli beam embedded in an elastic

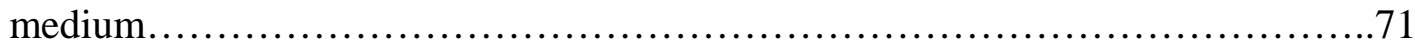

6.3.3. TWCNT modeled as nonlocal Euler-Bernoulli beam without elastic medium....75

6.3.4. TWCNT modeled as nonlocal Euler-Bernoulli beam embedded in an elastic medium. .78 


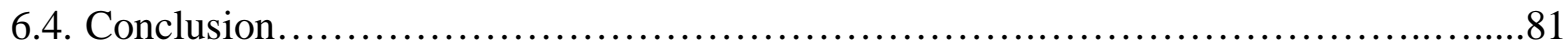

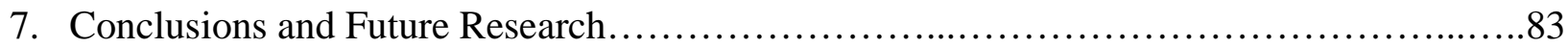

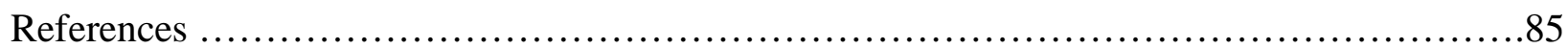

Appendices................................................................... 101 


\section{List of Tables}

Table 3.1 - FEM natural frequencies of DWCNTs, modeled as local Euler-Bernoulli beam.....25 Table 3.2 - Comparison of the nondimentionalized FEM natural frequencies of SWCNT with those of DWCNT, both modeled as local Euler-Bernoulli beam............................26 Table 3.3 - FEM natural frequencies of DWCNTs modeled as local Euler-Bernoulli beam,

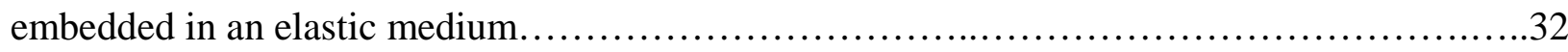
Table 4.1 - DSM natural frequencies of DWCNTs, modeled as local Euler-Bernoulli beam.....46 Table 4.2 - DSM natural frequencies of TWCNTs, modeled as local Euler-Bernoulli beam, embedded in an elastic medium.................................................... 47

Table 5.1 - FEM natural Frequencies of TWCNTs, modeled as local Euler-Bernoulli beam.....55 Table 5.2 - Comparison of the nondimentionalized FEM natural frequencies of a SWCNT with those of TWCNT, both modeled as local Euler-Bernoulli beam...............................55 Table 5.3 - FEM natural frequencies of TWCNTs modeled as local Euler-Bernoulli beam

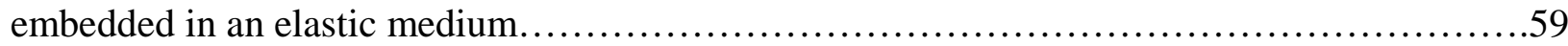
Table 6.1 - FEM natural frequencies of DWCNTs modeled as nonlocal Euler-Bernoulli beam.69 Table 6.2 - FEM natural frequencies of DWCNTs modeled as nonlocal Euler-Bernoulli beam embedded in an elastic medium..... .72 Table 6.3 - FEM natural frequencies of TWCNTs modeled as nonlocal Euler-Bernoulli beam..75 Table 6.4 - FEM natural frequencies of TWCNTs modeled as nonlocal Euler-Bernoulli beam embedded in an elastic medium. 


\section{List of Figures}

Figure 1a - Rolling of hexagonal graphite sheet to obtain carbon nanotubes..................11

Figure $1 \mathrm{~b}$ - Armchair, zig-zag and chiral configuration of carbon nanotube (L-R)..............12

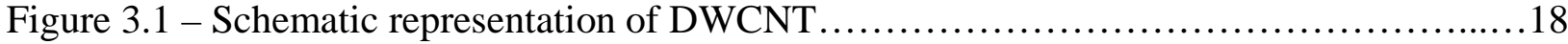

Figure 3.2 - Percentage error vs. the number of elements - SS - Up to 50 FEM elements........27

Figure 3.3 - Percentage error vs. the number of elements - CC - Up to 50 FEM elements.......27

Figure 3.4 - Percentage error vs. the number of elements - CF - Up to 50 FEM elements........28

Figure 3.5 - First five mode shapes of DWCNT modeled as local Euler-Bernoulli beam - simply supported boundary condition - FEM............................................ 29

Figure 3.6 - First two mode shapes of DWCNT modeled as local Euler-Bernoulli beam clamped-free boundary condition - FEM............................................

Figure 3.7 - First two mode shapes of DWCNT modeled as local Euler-Bernoulli beam clamped-clamped boundary condition - FEM.........................................

Figure 3.8 - Percentage error vs. the number of elements - embedded SS - Up to 50 FEM elements.

Figure 3.9 - Percentage error vs. the number of elements - embedded CC - Up to 50 FEM elements.

Figure 3.10 - Percentage error vs. the number of elements - embedded CF - Up to 40 FEM elements.

Figure 3.11 - First two mode shapes of DWCNT, modeled as local Euler-Bernoulli beam, embedded in elastic medium $(k / c=0.1)$ - simply supported boundary condition - FEM.......34 Figure 3.12 - First two mode shapes of DWCNT, modeled as local Euler-Bernoulli beam, embedded in elastic medium $(k / c=0.1)$ - clamped-free boundary condition - FEM...........35 Figure 3.13 - First two mode shapes of DWCNT, modeled as local Euler-Bernoulli beam, embedded in elastic medium $(k / c=0.1)$ - clamped-clamped boundary condition - FEM.......35

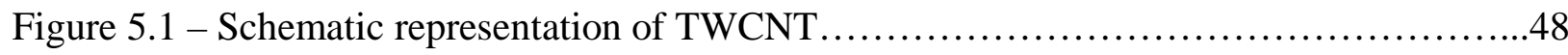
Figure 5.2 - Percentage error vs. the number of elements - SS - Up to 30 FEM elements.......56 Figure 5.3 - Percentage error vs. the number of elements - CC - Up to 30 FEM elements......56 Figure 5.4 - Percentage error vs. the number of elements - CF - Up to 30 FEM elements.......56 
Figure 5.5 - First two mode shapes of TWCNT modeled as local Euler-Bernoulli beam - simply supported boundary condition - FEM.

Figure 5.6 - First two mode shapes of TWCNT modeled as local Euler-Bernoulli beam clamped-free boundary condition - FEM.

Figure 5.7 - First two mode shapes of TWCNT modeled as local Euler-Bernoulli beam clamped-clamped boundary condition - FEM.... .58

Figure 5.8 - Percentage error vs. the number of elements - embedded SS - 30 FEM elements. .59

Figure 5.9 - Percentage error vs. the number of elements - embedded CC - 30 FEM elements.

Figure 5.10 - Percentage error vs. the number of elements - embedded CF - 30 FEM elements.

Figure 5.11 - First two mode shapes of TWCNT, modeled as local Euler-Bernoulli beam, embedded in elastic medium $(k / c=0.1)$ - simply supported boundary condition - FEM

Figure 5.12 - First two mode shapes of TWCNT, modeled as local Euler-Bernoulli beam, embedded in elastic medium $(k / c=0.1)$ - clamped-clamped boundary condition - FEM.......61 Figure 5.13 - First two mode shapes of TWCNT, modeled as local Euler-Bernoulli beam, embedded in elastic medium $(k / c=0.1)$ - clamped-free boundary condition - FEM..........62

Figure 6.1 - Percentage error vs. the number of elements - SS - Up to 50 FEM elements.......69 Figure 6.2 - Percentage error vs. the number of elements - CF - Up to 40 FEM elements......69 Figure 6.3 - Percentage error vs. the number of elements - CC - Up to 50 FEM elements......70 Figure 6.4 - First two mode shapes of DWCNT modeled as nonlocal Euler-Bernoulli - clamped free boundary condition - FEM................................................70

Figure 6.5 - First two mode shapes of DWCNT modeled as nonlocal Euler-Bernoulli - clampedclamped boundary condition - FEM................................................ 71 Figure 6.6 - First two mode shapes of DWCNT modeled as nonlocal Euler-Bernoulli - simply supported boundary condition - FEM............................................. 71 Figure 6.7 - Percentage error vs. the number of elements - embedded SS - Up to 50 FEM elements. 
Figure 6.8 - Percentage error vs. the number of elements -embedded CC - Up to 50 FEM

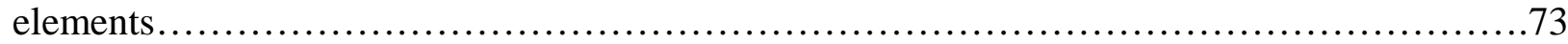

Figure 6.9 - Percentage error vs. the number of elements -embedded CF - Up to 50 FEM

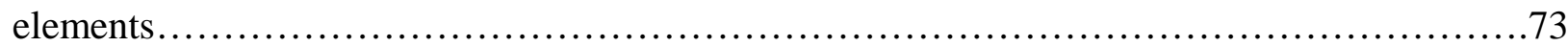

Figure 6.10 - First two mode shapes of DWCNT modeled as nonlocal Euler-Bernoulli - simply supported boundary condition - FEM........................................... 74

Figure 6.11 - First two mode shapes of DWCNT modeled as nonlocal Euler-Bernoulli clamped-clamped boundary condition - FEM........................................74

Figure 6.12 - First two mode shapes of DWCNT modeled as nonlocal Euler-Bernoulli clamped-free boundary condition - FEM........................................ 75

Figure 6.13 - Percentage error vs. the number of elements - SS - Up to 50 FEM elements.....76 Figure 6.14 - Percentage error vs. the number of elements - CC - Up to 50 FEM elements......76 Figure 6.15 - Percentage error vs. the number of elements - CF - Up to 40 FEM elements......76 Figure 6.16 - First two mode shapes of TWCNT modeled as nonlocal Euler-Bernoulli - simply supported boundary condition - FEM..............................................77 Figure 6.17 - First two mode shapes of TWCNT modeled as nonlocal Euler-Bernoulli clamped-clamped boundary condition - FEM.......................................77 Figure 6.18 - First two mode shapes of TWCNT modeled as nonlocal Euler-Bernoulli -

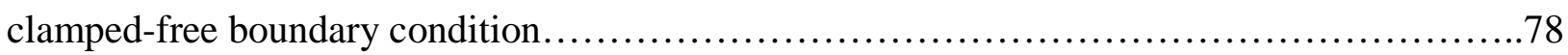
Figure 6.19 - Percentage error vs. the number of elements -embedded SS TWCNT - 50 FEM

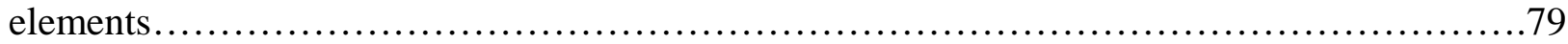
Figure 6.20 - Percentage error vs. the number of elements -embedded CC TWCNT - 50 FEM

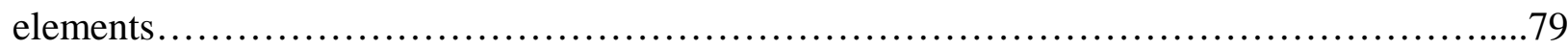
Figure 6.21 - Percentage error vs. the number of elements -embedded CF TWCNT - 50 FEM elements. .80

Figure 6.22 - First two mode shapes of TWCNT modeled as nonlocal Euler-Bernoulli, embedded in an elastic medium - clamped-free boundary condition - FEM..................80 Figure 6.23 - First two mode shapes of TWCNT modeled as nonlocal Euler-Bernoulli, embedded in an elastic medium - clamped-clamped boundary condition - FEM.

Figure 6.24 - First two mode shapes of TWCNT modeled as nonlocal Euler-Bernoulli, embedded in an elastic medium - simply supported boundary condition - FEM 


\section{Nomenclature}

$A$ - Cross-sectional area of the nanotube

E - Young's modulus

w - Displacement

$\rho$ - Mass density

$\sigma$ - Van der Waal's radius

$\varepsilon$ - Lennard-Jones potential’s Well Depth

$R_{1}$ - Inner radius

$R_{2}$ - Outer radius

$W_{k}$ - Transverse Displacements of $\mathrm{k}^{\text {th }}$ carbon nanotube

$\omega$ - Circular frequency

$t$ - Time

c - Van der Waal's interaction coefficient

$\delta W_{k}$ - Weighting function of transverse displacements of $\mathrm{k}^{\text {th }} \mathrm{CNT}$.

$k$ - Element stiffness matrix

$m$ - Mass matrix

$K$ - Global stiffness matrix

$M$ - Global mass matrix 


\section{List of Appendices}

A1 - Governing Equation for DWCNTs embedded in an elastic medium....................101

A2 - Stiffness and Mass matrices of DWCNTs............................................ 102

A3 - Mode shapes of DWCNTs embedded in elastic matrix...............................104

A4 - Expression for clamped-clamped natural frequency of DWCNTs....................106

A5 - Governing equations for TWCNT embedded in an elastic matrix......................107

A6 - Stiffness and mass matrices of TWCNTs........................................

A7 - Mode shapes of TWCNTs embedded in elastic matrix.............................110

A8 - Dynamic Stiffness Matrix (DSM) for TWCNTs.................................112

A9 - Dynamic Stiffness Matrix (DSM) for nonlocal DWCNTs.............................113 


\section{Introduction}

Carbon nanotubes have been under considerable observation and the subject of thousands of research publications every year, since their discovery. The global market for CNT primary grades is projected to reach $\$ 670.6$ million market in 2019 [1]. This interest can be attributed to their exceptional properties as a result of their geometry, namely, the high aspect ratio i.e., the ratio of its length to its diameter, and its chirality. These properties enable the use of carbon nanotubes in a wide variety of applications, some of which are electronics, sensors, composite materials, biotechnology, environment, optical, chemical, and energy storage. Therefore, in order to effectively use carbon nanotubes in each of these fields, it is important that their properties and behavior are examined.

In general, depending the property under observation, experimental or theoretical approaches are employed to study the properties and behavior of carbon nanotubes. Theoretical approaches are broadly categorised into atomistic modelling approach and continuum approach. The atomistic modelling approach, in turn, includes classical molecular dynamics approach, tight binding molecular approach and density functional approach [2]. Continuum approaches assume carbon nanotube to be continuous structures like bars, beams and shells. Though a great deal of research has been dedicated to finding the mechanical properties of carbon nanotubes using experimental and molecular dynamics methods, relatively less amount of research has been carried out on vibration and buckling of carbon nanotubes using these approaches, as the computational time and effort required is tremendous. Also, the results obtained would be valid for a very specific time period in the cycle of loading, thus limiting the exposure to their behavior. Therefore, continuum methods are used as they overcome these challenges. Yakobson et al. [3] mention that the application of continuum-elastic methods to carbon nanotubes predicts their behavior just as well and credits the laws of continuum mechanics of being applicable even at a scale so small. The current research is focussed on finding the natural frequencies and mode shapes of carbon nanotubes by considering them as local and non-local Euler-Bernoulli beams, exploiting the generality of finite elements method, a very often used approximate numerical method in analysis of dynamics of continuum structures, and dynamic stiffness method, an 'exact method' which harnesses the exact member theory and provides better accuracies for higher natural frequencies. 


\subsection{Outline of Finite Element Method (FEM)}

Finite element method (FEM) is probably one of the most well-known numerical methods used for structural analysis, with a proven track record owing to its adaptability - both in terms of complexity of structures and boundary conditions. It uses subdivision of the entire structure into smaller 'elements' governed by the relevant partial differential equations. Shape or interpolation functions (like Hermite cubic interpolation functions - used quite often for their completeness) are used to express the approximate solutions. The use of these shape functions in the partial differential equations gives elemental, and ultimately global, mass and stiffness matrices. These matrices are then solved as a linear eigenvalue problem to obtain the system's natural frequencies and modes. Finite element continuous to be used in different commercial packages directed towards structural analysis like ANSYS ${ }^{\circledR}$. The generality of this method stems from freedom in terms of defining the shape, boundary conditions, material properties of the structure and flexibility in terms of analysing complex structures which can include structures with different properties and/or different boundary conditions. The accuracy of FEM models can be considerably increased by increasing the number of elements being used. However for the analysis of structures at higher frequencies, finite element method has limited versatility, as it uses numerical approximation and large number of elements are required to obtain accurate results.

\subsection{Outline of Dynamic Stiffness Matrix (DSM) Method}

Dynamic Stiffness Matrix (DSM) method uses a single frequency dependent matrix, instead of two stiffness and mass matrices used in finite element method. The formulation involves, where applicable, combining the coupled governing differential equations into one ordinary differential equation and a closed form solution is sought. Application of boundary conditions to this solution leads to the dynamic stiffness matrix (DSM) of the system. The natural frequencies are obtained by solving resulting the non-linear eigenvalue problem. One of the biggest strength, and weakness of this method is the use of closed form solution of the differential equation. The closed form solution gives better accuracy, using a single element, even for higher frequencies but arriving at the closed form solution might prove to be a challenge, especially if the structure is complex and uses multiple structures with different properties and boundary conditions. An 
innovative technique, Dynamic Finite Element (DFE), combines the precision of this method with the generality of finite element method and has proven to give better results by using trigonometric shape functions, derived from the exact solution. Nonetheless, it is out of the scope of this thesis and hence is not presented in depth.

\subsection{Literature Review}

Vibration modelling and analysis of carbon nanotubes are important factors, eliciting extensive study, especially in applications such as oscillators and in non-destructive testing. The detection sensitivity of various nano-devices depends on their resonant frequency. Carbon nanotubes have considerably higher natural frequencies which could affect the system in which they are used and therefore it is required that their dynamic behavior is studied extensively. As mentioned earlier, continuum approach is preferred for studying the vibrational characteristics but a new hybrid technique, which links atomistic and continuum approaches, has also gained ground in the recent years. Odegard et al. [4] equated the molecular potential energy of nanomaterial with the strain energy of the continuum structure. Li and Chou christened this approach as 'molecular structural mechanics approach' and studied the buckling of carbon nanotubes [5], natural frequencies of single-walled carbon nanotube modeled as nano-mechanical resonator [6] and multi-walled carbon nanotubes based nanomechanical resonators [7]. They modeled carbon nanotube as frame-like structure with carbon-carbon bonds as nodes. A review of their work was presented in 2006 [8]. Chandrasekhar et al. [9] and Hollerer and Celigoj [10] both employed mixed approach by forming the constitutive model using atomistic approach and parameterization of continuum elastic energy for proposing a Cosserat rod model and for buckling analysis of carbon nanotubes respectively.

Continuum approach has seen the representation of carbon nanotubes as beams and shells for the most part. Very few studies like Hu et al. [11] and Budarapu et al. [12] studied vibrations of carbon nanotubes by considering them as continuum bars. Artan and Lehmann [13] used nonlocal bars to study the transverse vibrations of carbon nanotubes. On the other hand shells have gained comparatively more attention than bars. Ru [14] studied the infinitesimal buckling of a double-walled carbon nanotube and using simple elastic shell model for the same. Ru et al. 
[15] further studied the application of simplified shell equations by taking Donnel model, a simplified Flugge model to observe its applicability and limitations. Ru et al. [16] used multipleelastic-shell model to study the vibrations of multiwall carbon nanotubes employing Flugge's equations. Ansari et al. [17] used the shell elements in a hybrid approach to model single walled carbon nanotubes and compared it atomistic method. Ghorbanpour Arani et al. [18] used Donnel shell model to measure the transverse displacements of single and double-walled carbon nanotubes, the results of which are compared with Euler-Bernoulli and Timoshenko beam models. They observed that beam models predict the lowest frequency and shell models predict the highest. Pantano et al. [19] also used shell elements to effectively model and study the deformation of single and multiwall carbon nanotubes. Wang [20] studied the buckling and free vibration of multiwall carbon nanotubes using multiple-elastic-shell model. Natsuki et al. [21] studied the vibrational characteristics of fluid filled double-walled carbon nanotubes using simplified Flugge shell equations as the governing equations. Tylikowski [22] studied the thermally induced vibrations using layered-shell model also taking into account the van der Waals forces.

Eringen and Edelen [23], and Eringen [24,25] presented a theory, that takes into consideration the strain states at all points of the body for a particular stress state, called the nonlocal continuum mechanics theory, containing information about the long range forces in atoms, along which internal length scale is introduced as a material parameter. This theory was proven to be applicable to nanoscale by Peddieson et al. [26], where they applied it to nonlocal Euler-Bernoulli beam model. Zhang et al. [27] developed the multiple-shell model, based on the nonlocal continuum mechanics, to study the axial buckling of multiwall carbon nanotubes. Wang and Wang [28] presented the constitutive relation for small scale effects for Euler-Bernoulli and Timoshenko beams and for cylindrical shell problems. Hu et al. [29] investigated the transverse and torsional wave in single walled and double-walled carbon nanotubes modeled as nonlocal single and multiwall elastic shells. Wang and Varadan [30] used nonlocal elastic shell theory to study the wave propagation analysis of carbon nanotubes. Khademolhosseini et al. [31] studied the torsional buckling using nonlocal elasticity shell models. Khademolhosseini et al. [32] also studied the size effects in dynamic torsional response of single walled carbon nanotube by modelling it as a modified nonlocal continuum shell. Mikhasev [33] studied the vibrations of 
single walled carbon nanotubes embedded in nonhomogeneous elastic matrix using nonlocal continuum shell model. Recently Ansari et al. [34] investigated the free vibration characteristics of single and double-walled carbon nanotubes using nonlocal elastic shell model. Deng and Yang [35] studied vibration of fluid filled multiwall carbon nanotubes using nonlocal Flugge shell model. A rare approach of three dimensional vibrational and bending analysis was performed by Shaban and Alibeigloo [36] by taking nonlocal shell model to account for the size effect.

The continuum beam model has also been of considerable interest to study the dynamic properties of carbon nanotube. Ru [37] used the multiple column model to study its buckling and Ru et al. [38] used the classical Euler-Bernoulli beams for single wall and multiwall carbon nanotubes and studied their vibrations. Both these studies gave ample attention to interaction between the tubes which was represented using van der Waals forces. Yoon [39], in his doctoral thesis, did an extensive study on the use of beams for single wall and multiwall carbon nanotubes, using both Euler-Bernoulli and Timoshenko beams. He used analytical formulas to obtain the natural frequencies and to study wave propagation. Fu et al. [40] studied the nonlinear free vibration of multiwall carbon nanotubes embedded in an elastic medium using multiple elastic beam model. A similar study was performed by Yoon and Mioduchowski [41]. Natsuki et al. [42] studied the vibrational characteristics of a simply supported carbon nanotube based on Euler-Bernoulli beam. Natsuki et al. [43] also studied the vibration of double-walled carbon nanotubes, embedded in elastic medium and double-walled carbon nanotubes with different lengths for inner and outer nanotubes [44]. Wang et al. [45] used Timoshenko beam model to account for transverse shear and rotary inertia of multiwall carbon nanotubes. Wang et al. [46] and Shima [47] reviewed the buckling of carbon nanotubes. Ansari and Hemmatnezhad [48] studied the nonlinear vibrations of multiwall carbon nanotubes embedded in an elastic medium using variational iteration method (VIM). A similar study was performed by Sadri et al. [49] with the surrounding medium being modeled as Winkler type model. Cigeroglu and Samandari [50] employed differential quadrature method to perform yet another similar study. Ambrosini and Borbon [51] studied the influence of van der Waals interaction coefficient on the vibrations of double-walled carbon nanotubes using higher order beam model. Ansari and Hemmatnezhad [52] studied the vibrations of double-walled carbon nanotubes using nonlinear finite element analysis, where Von-Kamran type nonlinear strain-displacement relationships were employed to 
constraint the ends of carbon nanotubes to move axially. Baroudi and Razafimahery [53] studied the effects of an added mass on the natural frequency of clamped-clamped Euler-Bernoulli beam based carbon nanotube submerged in a fluid media. Vibrations of carbon nanotube-based composites were studied by Formica et al. [54] by employing a continuum model based on Eshelby-Mori-Tanaka approach. Adali [55] presented the variational principles for multiwall carbon nanotubes having nonlinear vibrations by considering the nonlinear geometric and physical effects arising due to large deflections and van der Waals forces between the tubes.

Nonlocal beam models have also been studied extensively in the recent years. Zhang et al. [56] studied the transverse vibrations of the double-walled carbon nanotubes using nonlocal Euler-Bernoulli beam model, for the first time. Tylikowski [57] studied instability of thermal vibrations of the single walled carbon nanotubes embedded in viscoelastic matrix under timedependent temperature field using nonlocal Euler-Bernoulli beam theory. Ansari and Hemmatnezhad [58] studied the vibration of embedded double-walled carbon nanotube modeled as nonlocal Timoshenko beam using energy equation approach and finite element method. A similar study was conducted by Shakouri et al. [59], considering nonlocal Euler-Bernoulli and using the Galerkin-type finite element methods. An early comparison between both these nonlocal approaches was performed by Wang [60]. Reddy [61] reformulated all the available beam theories using nonlocal relations and gave analytical solutions for bending, buckling and vibrations for each case. Ponnusamy and Amuthalakshmi [62] studied the vibrational characteristics of viscous fluid conveying double-walled carbon nanotube for clamped-clamped and clamped-free conditions. Wang and Wang [63] used nonlocal Timoshenko beam theory to study the vibration of carbon nanotubes embedded in elastic medium.

The current study considers carbon nanotubes to be Euler-Bernoulli beams, as local or crosssectional deformations (better studied using shell models), are not observed. Also, the high aspect ratio of the CNTs allow them to be reasonably represented as beams. To the best of author's knowledge, detailed Finite element formulations for double- and triple-walled carbon nanotubes, using Galerkin weighted residual approach, for local and nonlocal Euler-Bernoulli beam, has not been explicitly reported in the open literature. The use of this approach is important for the current study, as an extension of this thesis would aim to exploit the same principle to develop a Dynamic Finite Element (DFE) [64-73] which combines the generality of 
finite element method with the accuracy of Dynamic Stiffness Method (DSM). Dynamic Stiffness Method (DSM), as mentioned in the earlier section, combines the coupled equations into a single equation, the closed form solution of which gives the displacement and force matrices, which are then exploited to achieve dynamic stiffness matrix, to which the system's boundary conditions are applied. A brief yet simple explanation of the DSM formulation is given by Banerjee in reference [74]. This method has been used extensively due to the exactness it offers. Some of these studies include the free vibration of coupled bending-torsion Timoshenko beam element [75], coupled bending-torsion axially loaded Timoshenko beam element [76], twisted Timoshenko beam [77], composite Timoshenko beam [78], axially loaded composite Timoshenko beam [79], bending-torsion coupled beam with warping [80], twisted beam [81], sandwich beams [82], tapered beam [83], elastically connected three beam system [84] and axially loaded double beam system [85]. More recently, dynamic stiffness matrix for a general plate has also been formulated and presented by Banerjee et al. [86]. All these formulations use Wittrick-Williams (W-W) root counting algorithm [87] to find the number of roots below a chosen frequency and exploit a suitable (e.g., bisection) method to arrive at the system's targeted natural frequency. This thesis presents a Dynamic Stiffness Matrix (DSM) formulation, developed and used to find the natural frequencies of carbon nanotubes, for the first time.

\subsection{Objective}

The objective of this research is to study the free vibration characteristics of double-walled and triple-walled carbon nanotubes using analytical and approximate methods, considering them to be continuum structures, thereby preparing a foundation for vibrational study of complex structures that employ carbon nanotubes.

\subsection{Scope of Research}

This research is intended to develop the FEM formulations for the free vibration analysis of double- and triple-walled carbon nanotubes (CNTs), considering them as Euler-Bernoulli beams, using Galerkin weighted residual approach. This is presented for two cases, with and without an 
elastic medium surrounding the carbon nanotubes. This simple but important step prepares foundation for the development of dynamic stiffness matrix (DSM), which is formulated for double-walled carbon nanotubes for both the cases. Finite element formulation is also presented for double- and triple-walled carbon nanotubes based on nonlocal Euler-Bernoulli beam. This is also done for both cases, with and without an elastic medium surrounding them. By comparing the results for different cases, the effect of van der Waals interaction coefficient is studied. Also, the natural frequencies obtained using different analytical and finite element methods are compared to study the convergence rate for carbon nanotubes. The detailed Finite element formulations for double- and triple-walled carbon nanotubes, using Galerkin weighted residual approach, both for local and nonlocal Euler-Bernoulli beam, have been explicitly presented for the first time, and so has been the dynamic stiffness matrix (DSM) for double-walled CNTs.

\subsection{Organization of thesis}

The following chapters are arranged in such a way that a brief introduction to carbon nanotubes with history, properties, methods of synthesis, applications, in general, and for aerospace field in particular, are presented in Chapter 2.

Chapters 3 and 5 present the conventional finite element method (FEM) formulation for double and triple-walled carbon nanotubes using local Euler-Bernoulli beams, respectively, for both the cases with and without a surrounding elastic medium. The natural frequencies for these cases are found by solving the linear eigenvalue problem.

The dynamic stiffness matrix (DSM) formulation for double-walled carbon nanotubes is

presented in Chapter 4. Williams-Wittrick (W-W) root counting algorithm, used to solve the nonlinear eigenvalue problem, is also briefly discussed.

Chapter 6 presents the Galerkin weighted residual approach based finite element method for double and triple-walled carbon nanotubes based on nonlocal Euler-Bernoulli beams.

Finally conclusions and the path for future work is presented in Chapter 7. 


\section{Carbon Nanotubes - A Brief Introduction}

Carbon nanotubes (CNTs) continue to be a topic of considerable research in different areas of applications. The unique geometry of CNTs has been a key factor that gives them the rare properties they possess. A few of these properties and applications that benefit from those properties are discussed below. A brief historical outlook is provided and a section is dedicated to the CNTs' applications in aerospace engineering.

\subsection{History}

Carbon, in the graphitic form, was discovered in 1779 and in the form of diamond about 10 years later [88], having $s p^{2}$ and $s p^{3}$ bonding, respectively i.e., each carbon atom covalently bonded with 3 carbon atoms for graphite and with 4 carbon atoms for diamond. The following two centuries saw intensive study and application of carbon and was thought of a mature field until a new form of carbon was discovered. Although a good number of literature credits Iijima [89] with the discovery of carbon nanotubes, Boehm [90] mentioned that carbon nanotubes were observed much earlier than 1991, but in different structural form ("usually bent or coiled" [90]). They were reportedly discovered in 1952 by Radushekevich and Lukyanovich [91] and reported in Journal of Physical Chemistry, according to Boehm [90]. It was due to the geopolitical situation of the time that this discovery wasn’t popular among researchers in the West. Later, in 1955, Hofer et al. studied the carbon deposits on iron, nickel and cobalt, which were in form of "filaments from $0.01 \mu$ to $0.2 \mu$ in diameter" [92] (as action of carbon monoxide). In the following year, 1956, Richard Feynman opened researchers to the idea of "manipulating and controlling things on a small scale" in his now famous talk "Plenty of Room at the Bottom" [93] to American Physical Society. The following years saw the formation of graphitic whiskers [94], rolling over of graphite sheets, in a form now known as multiwall-carbon nanotubes (MWCNTs) [95]. Growth of carbon filaments in tubular, twisted and balloon forms [96], led to the use of the term 'nano-technology' for the first time [97] and its popularization through the 1980s [98]. Growth of hollow carbon fiber with large diameter in the direction of fiber axis was observed and reported by Oberlin et al. [99]. Research in carbon was invigorated with the discovery of fullerenes in 1985, which are closed cage-like structures of carbon with 60, 70 or 80 molecules 
arranged in symmetry to form $C_{60}, C_{70}$ and $C_{80}$ as polygons [100]. It was later in 1990 that Iijima [89], while studying $C_{60}$ in transmission electron microscope (TEM), discovered unusually tubular geometry of carbon molecules in carbon soot, later christened as carbon nanotubes. Iijima reported that these tubes have multiple graphitic sheets, ranging from 2 to 50, arranged coaxially, like Russian dolls, thus indicating that these were MWCNTs. Subsequently, Ebbessen and Ajayan [101] found efficient methods to make MWCNTs in gram quantities, using a variation of arc-discharge technique. It was in 1993 that Iijima and Ichihashi [102], and Bethune et al. [103], independently, found an efficient way of synthesizing single walled carbon nanotubes (SWCNTs). The following decades saw a surge in the volume of research dedicated to this field of nanotechnology, studying their exceptional properties arising due to its high length to diameter ratio and exploring their applications in a variety of fields, including but not limited to, micro-electronics, energy, mechanical, and biological applications.

\subsection{Structure}

A carbon nanotube is simply a graphite sheet rolled into a cylinder having very small diameter, in nanometer range, even as low as $0.4 \mathrm{~nm}$ [104], and lengths ranging from 10 nanometers to few centimeters, hence giving it high aspect ratio (length vs diameter). In case of a MWCNT, these are several nested cylinders with an interlayer spacing. These tubes exhibit a spiral conformation, called chirality, whose vector is given as $C_{h}=m a_{1}+n a_{2}$ where $a_{1}$ and $a_{2}$ are unit vectors in two-dimensional hexagonal lattice and the integer pair $(m, n)$ represents the translation indices. Taking these indices, the diameter of a single walled carbon nanotube (SWCNT) is given by:

$$
d=\frac{\left|\bar{C}_{h}\right|}{\pi}=0.0783 \sqrt{n^{2}+n m+m^{2}}
$$

and

$$
\theta=\sin ^{-1}\left[\frac{\sqrt{3} m}{2 \sqrt{n^{2}+n m+m^{2}}}\right]
$$


where $\theta$ is the angle of chirality i.e., the angle between $C_{h}$ and $a_{1}$ as shown in Figure 1 [46]. There are three cases depending on the chirality:

i) zig-zag configuration - $\theta=0$ and either $m$ or $n=0$;

ii) chiral configuration $-0^{\circ}<\theta<30^{\circ}$ and $m>n$;

iii) armchair configuration - $\theta=30^{\circ}$ and $m=n$;

For a MWCNT, two or more graphite sheets are rolled with different chirality angles [89,3,105]. For example, if the innermost nanotube is of zig-zag configuration, the rest are usually not. The interlayer spacing in MWCNT is close to the spacing for graphitic layers of $0.335 \mathrm{~nm}$ and is generally taken as $0.34 \mathrm{~nm}[88,105,106]$. The interaction between individual SWCNTs in a MWCNTs is via van der Waals (vdW) forces [106,108].

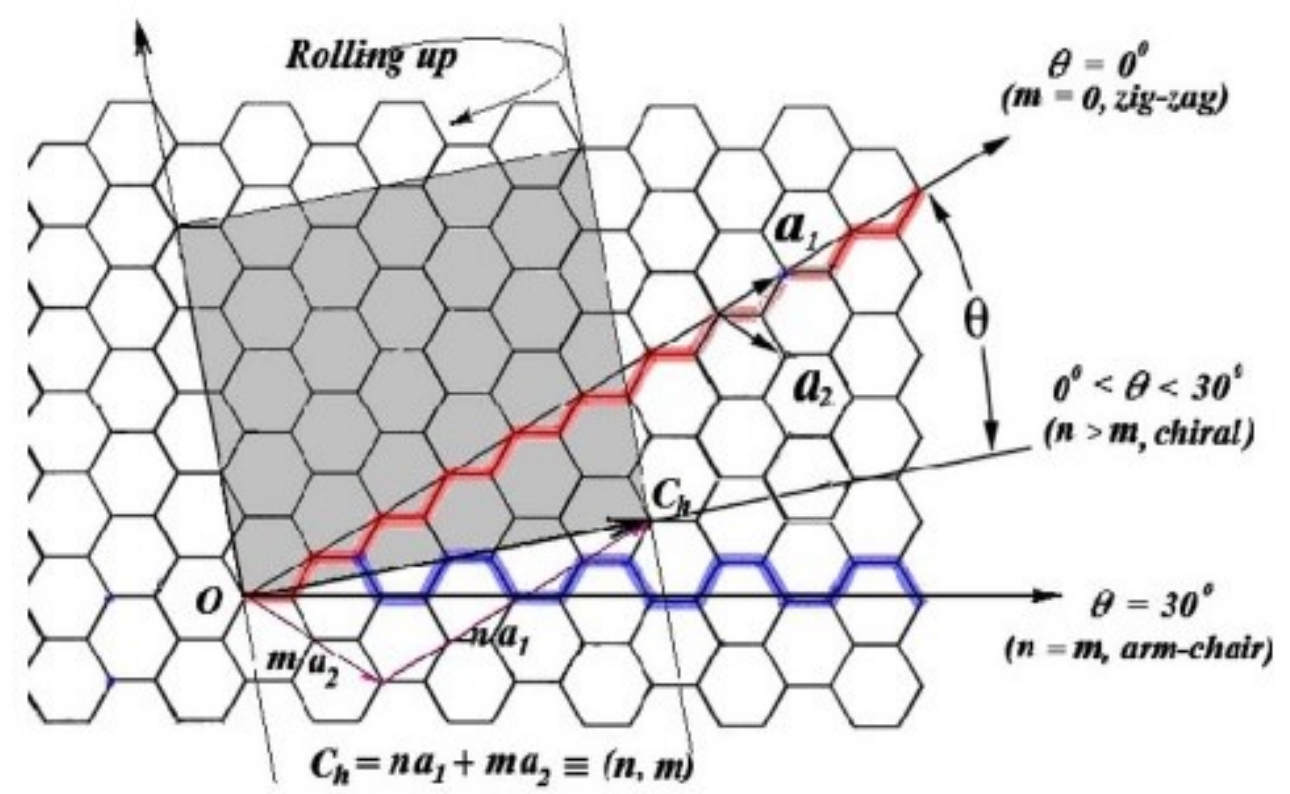

(a)

Figure 1a-Rolling of hexagonal graphite sheet to obtain carbon nanotubes [46] 

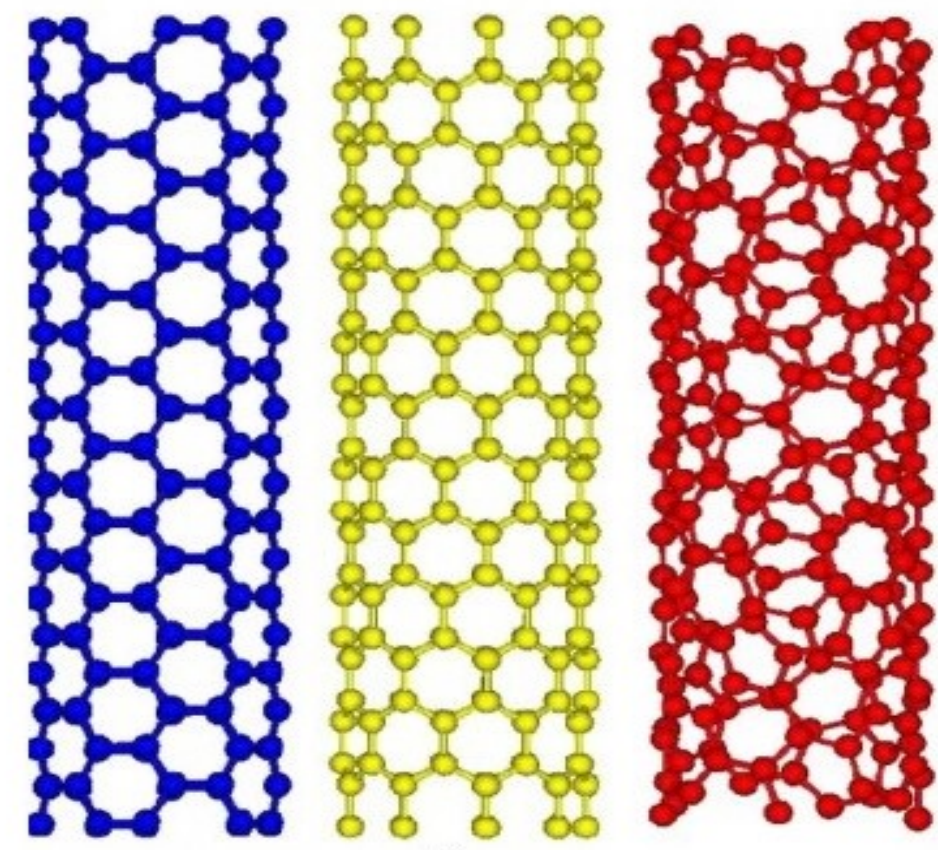

(b)

Figure $1 b$-Armchair, zig-zag and chiral configuration of carbon nanotube (L-R) [46]

\subsection{Synthesis}

Carbon nanotubes are mostly synthesized using three methods: Arc-discharge [109,110], laser ablation [111,112], and chemical vapor deposition [113,114].

2.3.1 Arc Discharge Method: Iijima [89] was using this method when carbon nanotubes were discovered in the carbon soot. This method involves the vaporization of carbon, under an inert gas in presence of a catalyst, which then condenses on the walls of the vessel and on the cathode. SWCNTs and MWCNTs are formed by adjusting the catalyst and pressure and are found on the cathode. This method is the widely popular as it gives the best quality carbon nanotubes.

2.3.2 Laser Ablation Method: Smalley et al. [111] used this method for the first time, in which the carbon nanotubes were in the soot on the cold end of the furnace which had a laser impinging on a graphite target. This method can only produce SWCNTs of controllable diameter. 
2.3.3 Chemical Vapor Deposition: This method involves a substrate coated with catalyst particles. This step guides the diameter of the carbon nanotubes, as it is dependent on the size of the clusters of catalyst particles on the substrate. The next step would involve a carboncontaining gas being carried in the reactor zone, which would decompose the carbon rich gas and carbon nanotubes are formed on the surface of the catalyst particle. Although this method doesn't give the best quality, it can be scaled up for production of large volumes of carbon nanotubes [115].

Schematic diagrams for above-mentioned methods can be found in Liu [116]. Apart from these, a few methods like spray pyrolysis [117-120] are also used. While the demand for carbon nanotubes is expected to rise to more than 153,000 tons by 2020 [121], the challenges faced by the industry for production include slow production rate, poor yield, inconsistency in material quality, no control over the production process and limited structural enhancements in bulk carbon nanotubes [122]. It is to be noted that manufacturing MWCNTs is much easier than SWCNTs, as indicated by the large quantities produced today [123].

\subsection{Properties}

The small size, chirality, $s p^{2}$ bond between carbon-carbon atoms, high aspect ratios and large surface areas are some of the major reasons for carbon nanotubes to have exceptional properties. For instance, their mechanical strength is 100 times more than that of steel at $1 / 6^{\text {th }}$ of weight. Some of the mechanical, electronic and thermal properties are briefly discussed below.

2.4.1 Mechanical Properties: The $s p^{2}$ carbon-carbon bond, in a carbon nanotube, means that each carbon is bonded with three other carbons and this gives unmatchable mechanical properties of carbon nanotubes. Carbon nanotubes are considered as structural members to find the elastic properties of a carbon nanotubes, both as SWCNT and a MWCNT. Treacy et al. [124] considered carbon nanotubes to be a clamped cylinder and found the average Young's modulus of a MWCNT to be $1.8 \mathrm{TPa}$. They analyzed the thermal vibration by TEM. Kazumaki et al. [125] performed an in-situ observation of the deformation of a CNT and report that it undergoes elastic deformation to a certain critical curvature point after which the atomic bonding 
changes to a diamond like state. A similar in-situ TEM study was performed by Wang et al. [126] to study electrical, mechanical, and field emission properties of carbon nanotubes. Wong et al. [127] considered CNTs to be a cantilever nanobeam and found the Young's modulus to be 660 GPa for SWCNT, and 1.28 \pm 0.59 TPa for MWCNTs. Similar studies were conducted by Krishnan et al. [128], Salvetat et al. [129, 130], Poncharal et al. [131], Lourie et al. [132] and it was found that the Young's modulus is always above $1 \mathrm{TPa}$. Salvetat et al. [130] reported that the elastic properties of the carbon nanotubes are rarely affected by the presence of point defects. Li et al. [133] extrapolated the tensile strength of SWCNTs bundles from the mini-composite and reported it to be in the range of $2.3 \pm 0.2$ to $14.2 \pm 1.4 \mathrm{GPa}$. The tensile strength of individual carbon nanotube is reported to be as high as 22.2 $\pm 0.2 \mathrm{GPa}$. Wei et al. [134] used molecular dynamics simulation and report the tensile strength of SWCNTs ropes and MWCNTs to be around 40-50 GPa. Yu et al. [132] recorded the tensile strengths of individual MWCNTs in the range of 11 to $63 \mathrm{GPa}$ for 19 MWCNTs. They also reported the "sword in sheath" failure i.e., the outermost layer broke in MWCNTs exhibiting the given tensile strength. Buongiorno et al. [135] reported that for carbon nanotubes deformation larger than 5\%, the system uses reversible formation of topological defects, releasing the excess strain. Belytschko et al. [136] reported the failure stress to be in the range of 93.5 to $112 \mathrm{GPa}$, and that carbon nanotubes undergo brittle failure and their chirality has a moderate effect on their strength. Yakobson et al. [137] studied the nanotube behavior at high tensile strain and reported extremely large breaking strain which reduces with the increase in temperature and has little influence of the carbon nanotube helicity. One of the in-depth reviews of the mechanical properties of carbon nanotubes, has been performed by Shokrieh and Rafiee [138] and aspects requiring further attention has been identified.

2.4.2 Electronic Properties: Carbon nanotubes can be either metallic or semiconducting depending on a lot of geometric differences such as chirality factor and diameter $[139,140]$. Ebbesen et al. [141] studied the electrical conductivity of MWCNTs by considering four probe measurements. They report that both metallic and non-metallic behavior are observed, in different tubes of the same MWCNT, along with sudden increase in the conductivity as temperature is increased. Tans et al. [142] reported that SWCNTs act as quantum wires based on the electrical transport measurements, whereas Delaney et al. [143] reported that MWCNTs have 
diffusive properties due to the presence of intertube interaction. Li et al. [144] studied the transport properties of $0.4 \mathrm{~cm}$ long carbon nanotubes and reported that they can be used in circuits as the transport properties are extensible even on $\mathrm{cm}$ scale lengths for nanotubes. Plombon et al. [145] studied the kinetic inductance of individual single-walled carbon nanotubes and high-frequency impedance of carbon nanotubes bundles and report that inductance of the bundle is scaled with the number of individual nanotubes. Cha et al. [146] prepared a bulk of randomly oriented carbon nanotubes and crosslinked them with each other through chemical reactions. They reported that the electric conductivity is increased by at least one order due to presence of crosslinks acting as bridges with low activation energy. Nihei et al. [147] studied the electrical properties of carbon nanotubes as interconnects between copper wiring. They observed that the electrical resistance of the interconnect having about 1000 carbon nanotubes was at least three orders of magnitude less than that of one carbon nanotube. The electrical conductivity of SWCNT reinforced polyimide composites was studied, as a function of concentration of SWCNT, by Ounaies et al. [148]. They reported that concentration of SWCNT can be customized for the required conductivity of the magnitude.

2.4.3 Thermal Properties: The rolling of a graphite sheet, as is done for carbon nanotubes, reduces the 2 dimensional phonon structure into 1 dimensional which gives it thermal properties that need attention. Ruoff et al. [149] had suggested that the thermal conductivity of carbon nanotubes would be higher than any known material. Che et al. [150] studied it using molecular dynamics approach for SWCNTs and made a similar observation. Hone et al. [151] reported that thermal conductivity of carbon nanotubes in bulk, such as aligned SWCNTs, is greater than 200 $\mathrm{W} / \mathrm{m} \mathrm{K}$, which is much higher than that of diamond or graphite. A similar study for individual MWCNTs was performed by Kim et al. [152] who observed the thermal conductivity to be 3000 $\mathrm{W} / \mathrm{K}$. Both these studies reported the values at room temperature. The thermal conductance of $2.76 \mu \mathrm{m}$ long, single-walled CNTs, suspended individually, was studied by Yu et al. [153]. They observed that free path for phonons is an important factor that decides the thermal conductivity. Chang et al. [154] proved that Fourier's law of thermal conductance is violated for carbon nanotubes, which would mean that the phonons in the carbon nanotubes don't scatter even when the length of the nanotube is exceeded considerably thereby giving "extraordinary" thermal transport properties. Pop et al. [155] presented a method of finding the thermal conductivity of 
SWCNT from high-bias electrical measurements thus relating the temperature on thermal conductance.

\subsection{Applications}

The remarkable properties of carbon nanotubes provide considerable opportunities for their usage in multitude of applications. This might be attributed to the ever increasing demand of multifunctional, efficient materials at reasonable costs. De Volder et al. [156] reported that the current carbon nanotube production exceeded several thousand tons annually and pegged the number close to 4.6 kiloton/year. These production capacities indicate the commercial interest in carbon nanotubes to be used in various applications including, but are not limited to, composites $[157,158]$ as fillers, as sensors in nanomechanical applications [159,160], as biosensors [161,162] to detect biomolecules, as gas sensors [163], as nanocarriers for drugs [164], in carbohydrate conjugation [165], to manipulate genes in genetic engineering [166], as nanofillers in dental applications [104], in tracking of cells and delivering transfection agents in tissue engineering [167-168], in repair of tissue after brain damage in neurogeneration [169], in diagnostic tools for biomedical applications [170,171], as conductive improver in Lithium-ion batteries [172], in production of new composite materials by nanocoating [173], as nanoprobes in electromechanical applications [123], as actuators [174], as interconnects [175,176], and as electron sources [177].

Aerospace industry has always been on the lookout for lighter and stronger materials that can bear extreme mechanical and thermal conditions. Carbon nanotubes, with their properties fit the description quite well. Nanocomposites are one area where the CNTs are mostly employed in aerospace industry. National Aeronautics and Space Administration (NASA) had released a roadmap for the application of nanotechnology in aerospace industry in the year 2010 [178]. It had detailed the areas of Materials, Energy Generation and Storage, Propulsion and Electronics, as the major fields where nanotechnology can make a difference. This comprehensive document had detailed the applications in each of these areas and presented challenges faced by each area with the possible solutions in a timeframe. Simonis et al. [179] in 2006, presented the lightweight materials, safety, intelligence-guidance, protection, low energy, high-speed and 
adaptive structures, as major areas in aeronautics, where nanotechnology has a potential. It has also listed the potential areas, where nanotechnology can be used in satellites. Gibson [180], in his review on the recent research of multifunctional materials, showed that the addition of CNTs to the composite materials increases their multifunctionality. Donnel et al. [181,182] studied the impact of CNTs on the commercial heavy aircraft. Zhao et al. [183] studied the usage of carbon nanotubes in composite film for de-icing. Self-sensing is an important factor from the point of view of morphing structures. Chung [184] reviewed the self-sensing of damage in carbon fiber polymer-matrix composites by measuring the electrical resistance. The usage of CNTs has also been explored for space elevator, a system designed for space travel, a major component of which is a cable that extends from earth to space. Carbon nanotubes are considered to be the material which form the ribbon, as their light weight will not let it buckle under its own weight. Laubscher [185] stated that woven carbon nanotubes based composite fibers form an important sub-system in space elevator. Cohen et al. [186] studied the oscillations of space elevator ribbon and gave mathematical formulations to find the static deformation and elastic oscillations. Pugno [187] studied the strength of carbon nanotube-based space elevator cable and concluded that the actual strength of the cable would be reduced by $\sim 70 \%$ of theoretical estimation due to presence of defects. Pugno et al. [188] studied the stability of the track of space elevator and concluded that the track in itself is orbitally unstable, but attaching an adequately heavy satellite can stabilize it. CNTs are also used in aircraft wiring [189], propulsion [190], lightening protection [191] and stealth aircraft designs [192]. It is believed that even after finding so many applications of carbon nanotubes, in aerospace and elsewhere, their true potential has not been realized yet. 


\section{Finite Element Formulation - Double-walled Carbon Nanotubes}

\subsection{Introduction}

Double-walled carbon nanotubes are the most common multiwall-carbon nanotubes used. The present continuum approach uses local Euler-Bernoulli beam to represent individual carbon nanotube, which are coupled through the van der Waals forces. The following section gives the formulation using Galerkin weighted residual approach to form the mass and stiffness matrices. A schematic representation of DWCNTs is given in Figure 3.1.

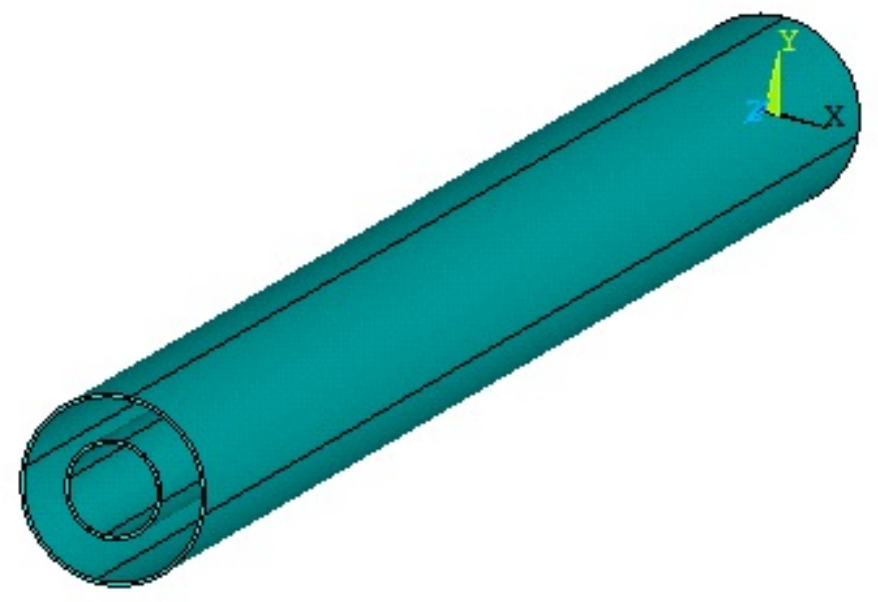

Figure 3.1 - Schematic representation of DWCNT

\subsection{Formulation}

As the elastic beam has been shown to represent the properties of a carbon nanotube efficiently, when the length of the tube is much larger than the diameter, the carbon nanotube is modeled as Euler-Bernoulli beam. If $w$ is the displacement, $\rho$ is the mass density, $A$ is the cross-sectional area and $E I$ is the bending rigidity of the beam, the governing differential equation takes the form [193]:

$$
E I w^{\prime \prime \prime \prime}+\rho A \ddot{w}=p(x)
$$

Prime (') and dot (.) represent derivatives with respect to the length of the beam and time, respectively. A double-walled carbon nanotube (DWCNT) is modeled as two single-walled 
carbon nanotubes coupled by an interaction pressure represented by van der Waal's coefficient, estimated as [193]:

$$
c=\frac{\pi \varepsilon R_{1} R_{2} \sigma^{6}}{a^{4}}\left[\frac{1001 \sigma^{6}}{3} H^{13}-\frac{1120}{9} H^{7}\right]
$$

where $\sigma$ and $\varepsilon$ are the van der Waal's radius and Lennard-Jones potential's well depth, repectively, $a=0.142$ is the C-C bond length, and $R_{1}$ and $R_{2}$ are the inner and outer radius respectively, whereas

$$
H^{m}=\left(R_{1}+R_{2}\right)^{-m} \int_{0}^{\frac{\pi}{2}} \frac{d \theta}{\left(1-K \cos ^{2} \theta\right)^{\frac{m^{\prime}}{2}}} \quad(\mathrm{~m}=7,13)
$$

and

$$
K=\frac{4 R_{1} R_{2}}{\left(R_{1}+R_{2}\right)^{2}}
$$

The governing equations for a DWCNT are then written as [38]:

$$
\begin{gathered}
E_{1} I_{1} w_{1}^{\prime \prime \prime \prime}+\rho_{1} A_{1} \ddot{w}_{1}=c w_{2}-c w_{1} \\
E_{2} I_{2} w_{2}^{\prime \prime \prime \prime}+\rho_{2} A_{2} \ddot{w}_{2}=-\hat{c} w_{2}+c w_{1}
\end{gathered}
$$

where the subscripts 1,2 for $E, I, w, \rho$, and $A$ represent the inner and outer tubes, respectively. $\hat{c}$ is used to denote two cases; DWCNT without any medium and DWCNT embedded in an elastic medium, respectively, as given in equations (3.7) and (3.8) below.

$$
\begin{gathered}
\hat{C}=c \\
\hat{c}=c+k
\end{gathered}
$$

where Winkler like model is considered to denote the pressure acting on the outermost tube due to the presence of elastic model. $k$ in (3.8) is dependent on the material constants of the elastic medium, the outer diameter of the embedded tubes and wavelength of vibrational modes, and is defined in terms of the van der Waals forces, $c$. The mathematical formulation to arrive at the corresponding equation is presented in Appendix A1. 
The time dependency in (3.5) and (3.6) is eliminated by considering simple harmonic motion and using the following transformations.

$$
\begin{aligned}
& w_{1}(x, t)=W_{1} e^{i \omega t} \\
& w_{2}(x, t)=W_{2} e^{i \omega t}
\end{aligned}
$$

where $W_{1}$ and $W_{2}$ are the amplitudes of transverse displacements of inner and outer carbon nanotube, respectively, and $\omega$ and $t$ represent the circular frequency and time, respectively. These transformations are substituted in the governing equations, (3.5) and (3.6) to obtain:

$$
\begin{aligned}
& E_{1} I_{1} W_{1}^{\prime \prime \prime \prime}+\left(c-\rho_{1} A_{1} \omega^{2}\right) W_{1}-c W_{2}=0 \\
& E_{2} I_{2} W_{2}^{\prime \prime \prime \prime}+\left(\hat{c}-\rho_{2} A_{2} \omega^{2}\right) W_{2}-c W_{1}=0
\end{aligned}
$$

The coupling between both beams is represented by the last terms in (3.11) and (3.12), the first beam has the van der Waal's interaction coefficient $c$ with the transverse displacement of the beam 2, shown in (3.11) by $W_{2}$ and same can be observed in (3.12). Taking the amplitude of displacements as $W_{1}$ and $W_{2}$, the Galerkin weighted residual method is applied on both equations with $\delta W_{1}$ and $\delta W_{2}$ as the weighting function, the integral form of (3.11) and (3.12) is developed as shown below.

$$
\begin{aligned}
& \bar{W}_{1}=\int_{0}^{L}\left(E_{1} I_{1} W^{\prime \prime \prime} \delta W_{1}+\left(c-\rho_{1} A_{1} \omega^{2}\right) W_{1} \delta W_{1}-c W_{2} \delta W_{1}\right) d x=0 \\
& \bar{W}_{2}=\int_{0}^{L}\left(E_{2} I_{2} W^{\prime \prime \prime \prime} \delta W_{2}+\left(\hat{c}-\rho_{2} A_{2} \omega^{2}\right) W_{2} \delta W_{2}-c W_{1} \delta W_{2}\right) d x=0
\end{aligned}
$$

The weighting functions $\delta W_{1}$ and $\delta W_{2}$ represent the transverse displacements of inner and outer carbon nanotubes, respectively. The weak integral form of the governing equations is developed by integrating (3.13) and (3.14) by parts, as given below:

$$
\begin{gathered}
\bar{W}_{1}=\int_{0}^{L}\left(E_{1} I_{1} W_{1}^{\prime \prime} \delta W_{1}^{\prime \prime}+\left(c-\rho_{1} A_{1} \omega^{2}\right) W_{1} \delta W_{1}-c W_{2} \delta W_{1}\right) d x+\left[\left(E_{1} I_{1} W^{\prime \prime \prime}\right) \delta W_{1}\right]_{0}^{L} \\
-\left[\left(E_{1} I_{1} W^{\prime \prime}\right) \delta W_{1}^{\prime}\right]_{0}^{L}=0
\end{gathered}
$$




$$
\begin{gathered}
\bar{W}_{2}=\int_{0}^{L}\left(E_{2} I_{2} W_{2}^{\prime \prime} \delta W_{2}^{\prime \prime}+\left(\hat{c}-\rho_{2} A_{2} \omega^{2}\right) W_{2} \delta W_{2}-c W_{1} \delta W_{2}\right) d x+\left[\left(E_{2} I_{2} W_{2}^{\prime \prime \prime}\right) \delta W_{2}\right]_{0}^{L} \\
-\left[\left(E_{2} I_{2} W^{\prime \prime}\right) \delta W_{2}^{\prime}\right]_{0}^{L}=0
\end{gathered}
$$

The boundary terms in (3.15) and (3.16) vanish with the application of system boundary conditions, hence the strikethrough. The system is then discretized using a number $(k)$ of 4-node and 2-DOF per node (one transverse displacement and one slope per node) such that

$$
\begin{aligned}
& \bar{W}_{w_{1}}^{k}=\int_{0}^{l^{k}} \delta W_{1}^{\prime \prime}\left(E_{1} I_{1} W_{1}^{\prime \prime}\right) d x+\int_{0}^{l^{k}} \delta W_{1}\left(c-\rho_{1} A_{1} \omega^{2}\right) W_{1}-\int_{0}^{l^{k}} \delta W_{1} c W_{2} \\
& \bar{W}_{w_{2}}^{k}=\int_{0}^{l^{k}} \delta W_{2}^{\prime \prime}\left(E_{2} I_{2} W_{2}^{\prime \prime}\right) d x+\int_{0}^{l^{k}} \delta W_{2}\left(\hat{c}-\rho_{2} A_{2} \omega^{2}\right) W_{2}-\int_{0}^{l^{k}} \delta W_{2} c W_{1}
\end{aligned}
$$

Hermite type polynomial approximations are used, as done in classical Euler-Bernoulli beam finite element development, for each carbon nanotube in (3.17) and (3.18). For a two-node, two-DOF per node element, the non-nodal displacement function is written as:

$$
W_{n}(x)=\left\langle 1 \quad x \quad x^{2} \quad x^{3}\right\rangle\left\{c_{n}\right\}(n=1,2)
$$

where $\left\{C_{n}\right\}(n=1,2)$ represents a column of unknown constant coefficients for inner and outer carbon nanotubes, respectively. The vector of nodal displacements for bending are given by:

$$
\left\{W_{n}\right\}=\left\{\begin{array}{l}
W_{1} \\
W_{1}^{\prime} \\
W_{2} \\
W_{2}^{\prime}
\end{array}\right\}=\left[\begin{array}{cccc}
1 & 0 & 0 & 0 \\
0 & 1 & 0 & 0 \\
1 & L & L^{2} & L^{3} \\
0 & 1 & 2 L & 3 L^{2}
\end{array}\right]\left\{c_{n}\right\}=\left[P_{n}\right]\left\{c_{n}\right\}
$$

Thus

$$
W_{n}(x)=\left\langle 1 \quad x \quad x^{2} \quad x^{3}\right\rangle\left[P_{n}\right]^{-1}\left\{W_{n}\right\}=\left\langle N_{i}\left(x_{i}\right)\right\rangle\left\{W_{n}\right\}
$$

where $\left\langle N_{i}\left(x_{i}\right)\right\rangle$ is a row vector of cubic shape functions, of the beam elements given by:

$$
\begin{aligned}
& N_{1}=\frac{1}{L^{3}}\left(2 x^{3}-3 x^{2} L+L^{3}\right) \\
& N_{2}=\frac{1}{L^{3}}\left(x^{3} L-2 x^{2} L+x L\right)
\end{aligned}
$$




$$
\begin{aligned}
& N_{3}=\frac{1}{L^{3}}\left(-2 x^{3}+3 x^{2} L\right) \\
& N_{4}=\frac{1}{L^{3}}\left(x^{3} L-x^{2} L^{2}\right)
\end{aligned}
$$

These shape functions are also used to approximate the virtual displacements:

$$
\delta W_{n}(x)=\left\langle N_{i}\left(x_{i}\right)\right\rangle\left\{\delta W_{n}\right\}
$$

Using the equations (3.20) through (3.22), the stiffness, mass, semi-mass and coupling matrices, represented by $k, m, s m$, and $\mathrm{cm}$ respectively, are given in equation (3.24). Also equations (3.17) and (3.18) are represented in form of uncoupled matrices as (3.25) and (3.26)

$$
\begin{aligned}
& k=\left(\begin{array}{cccc}
\frac{12 E I}{l^{3}} & \frac{6 l E I}{l^{3}} & \frac{-12 E I}{l^{3}} & \frac{6 l E I}{l^{3}} \\
\frac{4 l^{2} E I}{l^{3}} & \frac{-6 l E I}{l^{3}} & \frac{2 l^{2} E I}{l^{3}} \\
\text { Sym } & \frac{12 E I}{l^{3}} & \frac{-6 l E I}{l^{3}}
\end{array}\right) m=\left(\begin{array}{cccc}
\frac{156 m l}{420} & \frac{22 l^{2} m}{420} & \frac{54 m l}{420} & \frac{-13 l^{2} m}{420} \\
& \frac{4 l^{3} m}{420} & \frac{13 l^{2} m}{420} & \frac{-3 l^{3} m}{420} \\
& & & \frac{4 l^{2} E I}{l^{3}}
\end{array}\right) \\
& s m=\left(\begin{array}{cccc}
\frac{156 c}{420} & \frac{22 l c}{420} & \frac{54 c}{420} & \frac{-13 l c}{420} \\
\frac{4 l^{2} c}{420} & \frac{13 l c}{420} & \frac{-3 l^{2} c}{420} \\
\text { Sym } & \frac{156 c}{420} & \frac{-22 l c}{420} \\
& & & \frac{4 l^{2} c}{420}
\end{array}\right) \quad c m=\left(\begin{array}{cccc}
\frac{156 c}{420} & \frac{22 l c}{420} & \frac{54 c}{420} & \frac{-13 l c}{420} \\
& \frac{4 l^{2} c}{420} & \frac{13 l c}{420} & \frac{-3 l^{2} c}{420} \\
& S y m & \frac{156 c}{420} & \frac{-22 l c}{420} \\
& & & \frac{4 l^{2} c}{420}
\end{array}\right)
\end{aligned}
$$




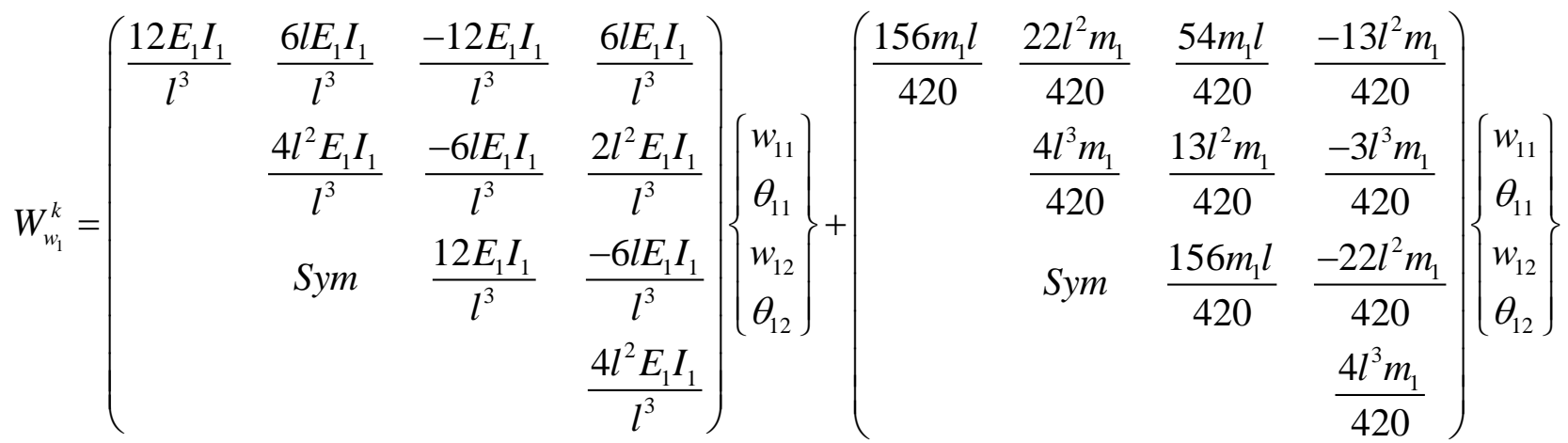

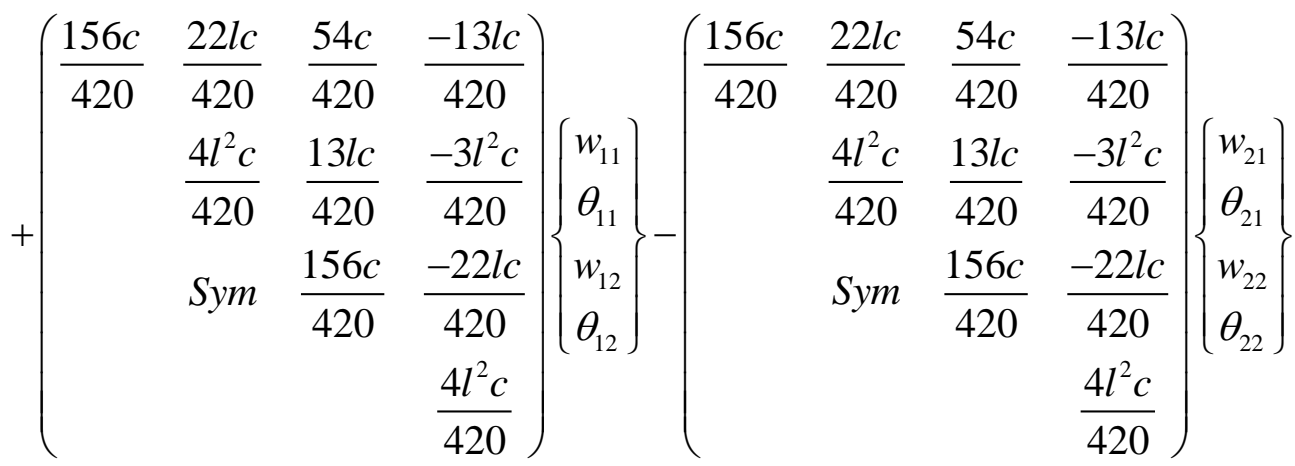

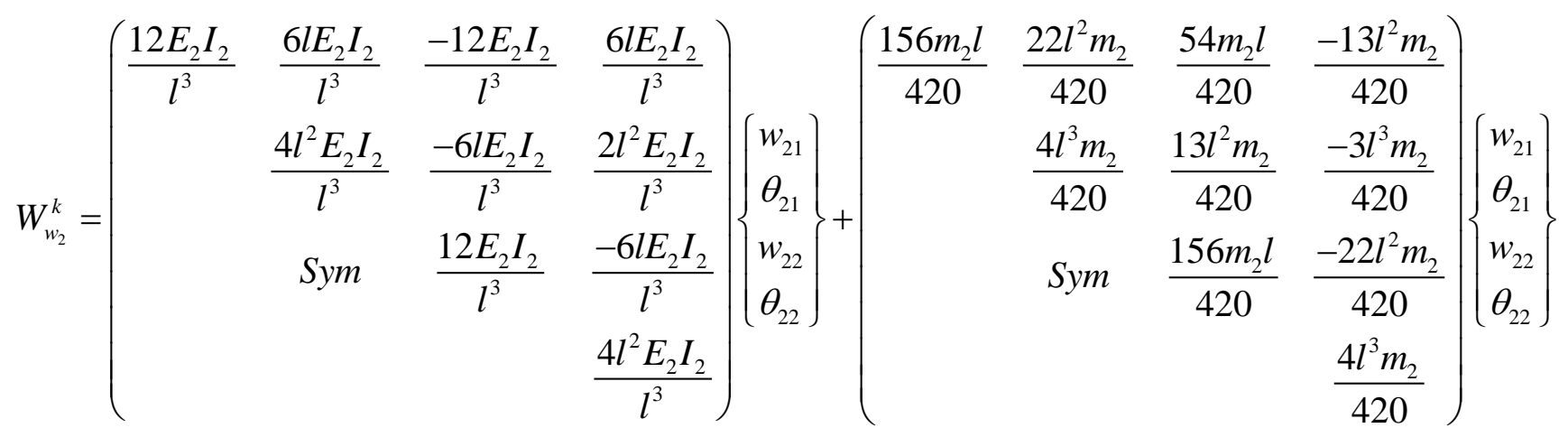

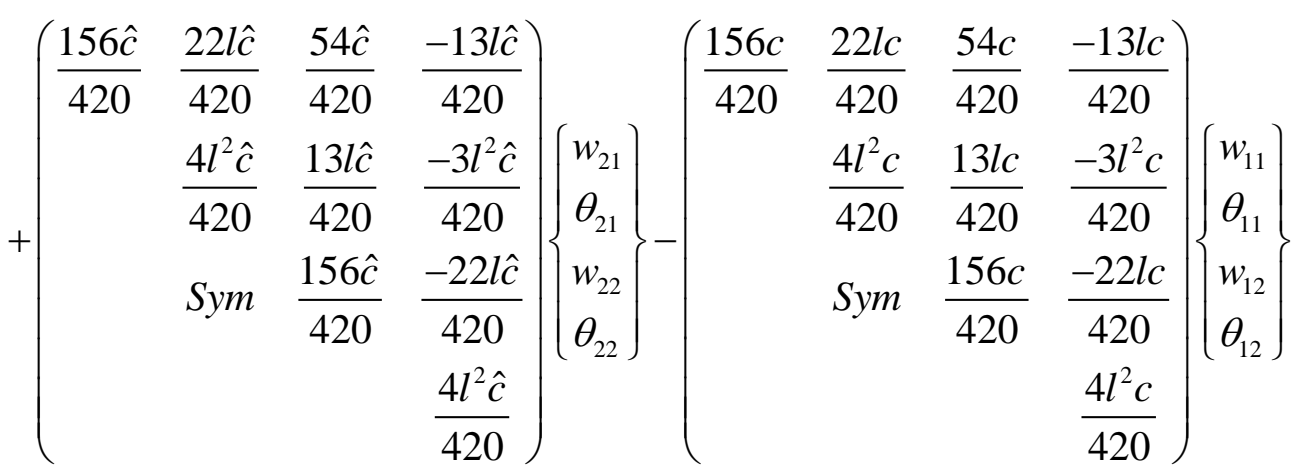

The element stiffness and mass matrices in the coupled form are represented as $[k]^{k}$ and $[m]^{k}$, respectively. The assembly of the element matrices and application of system's boundary 
condition is carried out using a FEM code written in MATLAB ${ }^{\circledR}$, leading to the system's linear eigenvalue problem written as:

$$
\begin{gathered}
\left\langle\delta W_{n}\right\rangle\left(K-\omega^{2} M\right)\left\{W_{n}\right\}=0 \\
\operatorname{det}\left(K-\omega^{2} M\right)=0
\end{gathered}
$$

where $K$ represents global stiffness matrix and $M$ stands for the global mass matrix, both of which are $8 \times 8$ in size. The global matrices are defined as the collection of all the element matrices, i.e.,

$$
\bar{W}=\sum_{k=1}^{\text {No.ofelements }} \bar{W}_{1}^{k}+\bar{W}_{2}^{k}
$$

\subsection{Results and Discussion}

In this section, the application of the FEM formulation presented above is demonstrated through the free vibration analysis of illustrative CNT examples, where the following geometric and material properties and dimensions, reported by Xu et al. [195], are used:

$$
\begin{aligned}
& L=14 e-9 \text {, } \\
& \rho_{1}=\rho_{2}=2.3 \frac{g}{\mathrm{~cm}^{3}} \\
& E_{1}=E_{2}=1 T P a, \\
& c=71.11 G P a \\
& r_{1, i}(\text { inner radius, inner tube })=0.18 \mathrm{~nm}, \quad r_{1, o}(\text { outer radius, inner tube })=0.52 \mathrm{~nm} \text {, } \\
& r_{2, i}(\text { inner radius, outer tube })=0.53 \mathrm{~nm}, \quad r_{2, o}(\text { outer radius, outer tube })=0.87 \mathrm{~nm}
\end{aligned}
$$

\subsubsection{DWCNT modeled as local Euler-Bernoulli beam}

The natural frequencies for DWCNT with three different boundary conditions, namely, clamped-clamped (CC), clamped free (CF) and simply supported (SS), modeled as local EulerBernoulli beam, are tabulated in Tables 3.1. The natural frequencies computed in the present study, i.e., using finite element method (FEM), are found to be in agreement with the so-called exact results provided by Xu et al. [195], which were found by solving the coupled equations. 
The convergence of natural frequencies reported in this study, was verified and arrived at by changing the number of elements gradually from one to a point where the relative error becomes constant.

Table 3.1 also shows the percentage error between the FEM natural frequencies, exact values reported by Xu et al. [195], and two other approximate methods; i.e., results reported by Elishakoff et al. [196], obtained using Bubnov-Galerkin or Petrov-Galerkin methods. In the table, $\omega_{1}$ denotes the first natural frequency of the system whereas $\omega_{2}$ denotes the first noncoaxial natural frequency of the system.

In the present study, the FEM natural frequencies are obtained using a 50-element mesh, although some cases may converge using a less refined mesh. It can be seen that the error percentage between the present study and the exact values provided by Xu et al. [195] is at most in the order of $10^{-2}$, hence showing high rate of convergence.

Table 3.1 - FEM natural frequencies of DWCNTs, modeled as local Euler-Bernoulli beam

\begin{tabular}{|c|c|c|c|c|c|c|c|}
\hline \multirow{2}{*}{\multicolumn{2}{|c|}{$\begin{array}{l}\text { Boundary } \\
\text { Conditions }\end{array}$}} & \multirow{3}{*}{$\begin{array}{c}\begin{array}{c}\text { Xu et al. [195] } \\
\left(10^{12} \mathrm{rad} / \mathrm{s}\right)\end{array} \\
\begin{array}{c}\text { Natural } \\
\text { Frequencies }\end{array} \\
0.467\end{array}$} & \multicolumn{3}{|c|}{$\begin{array}{l}\text { Elishakoff et al. [196] } \\
\qquad\left(10^{12} \mathrm{rad} / \mathrm{s}\right)\end{array}$} & \multicolumn{2}{|c|}{$\begin{array}{c}\text { FEM } \\
\left(10^{12} \mathrm{rad} / \mathrm{s}\right) \\
\end{array}$} \\
\hline & & & Natural & Method & Percentage & $\begin{array}{c}\text { Natural } \\
\text { Freauencie }\end{array}$ & Percentag \\
\hline \multirow{2}{*}{$\begin{array}{c}\text { Simply } \\
\text { Supported }\end{array}$} & $\omega_{1}$ & & 0.467 & \multirow{2}{*}{$\begin{array}{l}\text { Petrov } \\
\text { Galerkin }\end{array}$} & $-0.11 \%$ & 0.467 & $0.00 \%$ \\
\hline & $\omega_{2}$ & 7.885 & 7.885 & & $-0.00 \%$ & 7.885 & $0.00 \%$ \\
\hline \multirow{2}{*}{$\begin{array}{l}\text { Clamped } \\
\text { Clamped }\end{array}$} & $\omega_{1}$ & 1.058 & 1.049 & \multirow{2}{*}{$\begin{array}{l}\text { Petrov } \\
\text { Galerkin }\end{array}$} & $0.80 \%$ & 1.058 & $0.00 \%$ \\
\hline & $\omega_{2}$ & 7.925 & 7.924 & & $0.01 \%$ & 7.925 & $0.00 \%$ \\
\hline \multirow{2}{*}{$\begin{array}{l}\text { Clamped } \\
\text { Free }\end{array}$} & $\omega_{1}$ & 0.166 & 0.167 & \multirow{2}{*}{$\begin{array}{l}\text { Bubnov } \\
\text { Galerkin }\end{array}$} & $-0.41 \%$ & 0.166 & $0.01 \%$ \\
\hline & $\omega_{2}$ & 7.877 & 7.877 & & $-0.00 \%$ & 7.877 & $0.00 \%$ \\
\hline
\end{tabular}

A comparison of nondimensionalized natural frequencies of individual tubes of a DWCNT, and of DWCNT by using a single beam model is presented in Table 3.2. The equivalent single beam 
model uses the geometric properties obtained as a sum of properties of individual tubes, as explained below. Taking the Euler-Bernoulli beam equation:

$$
E I w^{\prime \prime \prime}+\rho A \ddot{w}=0
$$

with $I=I_{1}+I_{2}$ and $A=A_{1}+A_{2}$ with $I_{1}, I_{2}$ and $A_{1}, A_{2}$ are moments of inertias and areas of each tube [41]. This method is particularly useful to understand the effect van der Waals interaction coefficient has on the natural frequency of a DWCNT, as shown by the difference in Table 3.2. The nondimentional natural frequency is given by

$$
\mu=\sqrt{\frac{m}{E I}} L^{2} \omega
$$

Table 3.2 - Comparison of the nondimentionalized FEM natural frequencies of SWCNT with those of DWCNT, both modeled as local Euler-Bernoulli beam

\begin{tabular}{|c|c|c|c|}
\hline \multirow{2}{*}{$\begin{array}{c}\text { Boundary } \\
\text { Conditions }\end{array}$} & $\begin{array}{c}\text { DWCNT } \\
\text { (Inner Tube) }\end{array}$ & $\begin{array}{c}\text { DWCNT } \\
\text { (Outer Tube) }\end{array}$ & $\begin{array}{c}\text { Single Beam } \\
\text { Model }\end{array}$ \\
\cline { 2 - 4 } & $\omega_{11}$ & $\omega_{12}$ & $\omega$ \\
\hline Simply Supported & 15.9646 & 145.5142 & 9.8698 \\
\hline Clamped-Clamped & 36.1391 & 146.2479 & 22.3752 \\
\hline Clamped Free & 5.6890 & 145.3596 & 3.5161 \\
\hline
\end{tabular}

It can be seen from Table 3.2 that the nondimentionalized frequency of the inner tube of a local Euler-Bernoulli based beam, obtained, using (3.30), is always lower than that of the outer beam, owing to smaller geometry. The natural frequency obtained by the single beam model gives the smallest value for all the three boundary conditions surveyed. It is to be noted that the nondimentional natural frequency of the multi-walled carbon nanotube system would require the equivalent Young's modulus and moment of inertia and is not a part of this thesis. In finite element analysis (FEA), it is important that an optimum number of elements are considered for 
obtaining a converged value. Any number of elements less than that particular number can undermine the natural frequency value and anything more could result in errors arising out of extensive mathematical manipulations. One way to check the convergence is by observing the error percentage. When the error percentage goes towards zero or stabilises, it is said that the value is converged, like the ones presented in Table 3.1. The graphs showing convergence are plotted below, Figures (3.2) - (3.4). The percentage error was computed with respect to the exact values as given by Xu et al. [195] and has been plotted versus number of elements, in the figures. The number of elements are increased to the point where the error percentage becomes stable signaling the convergence of natural frequency.

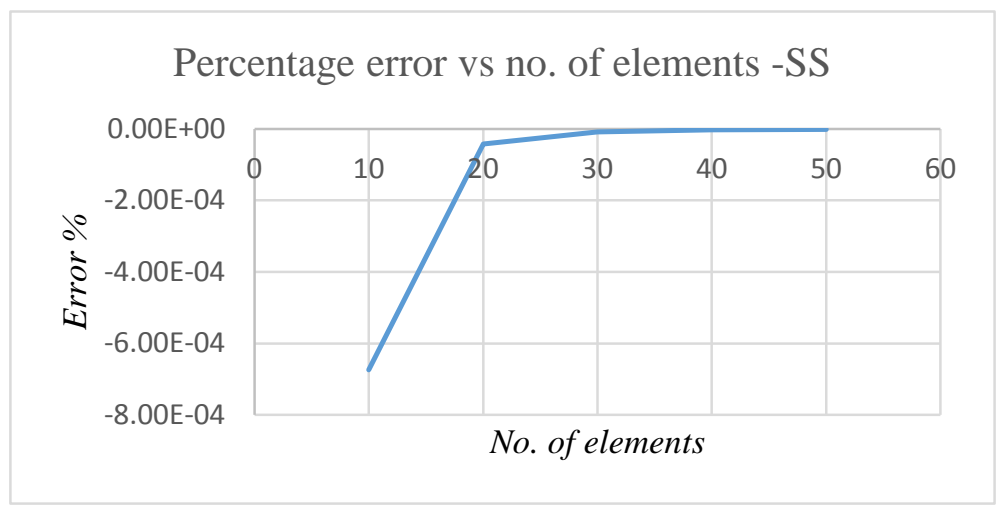

Figure 3.2 - Percentage error vs. the number of elements - SS - Up to 50 FEM elements

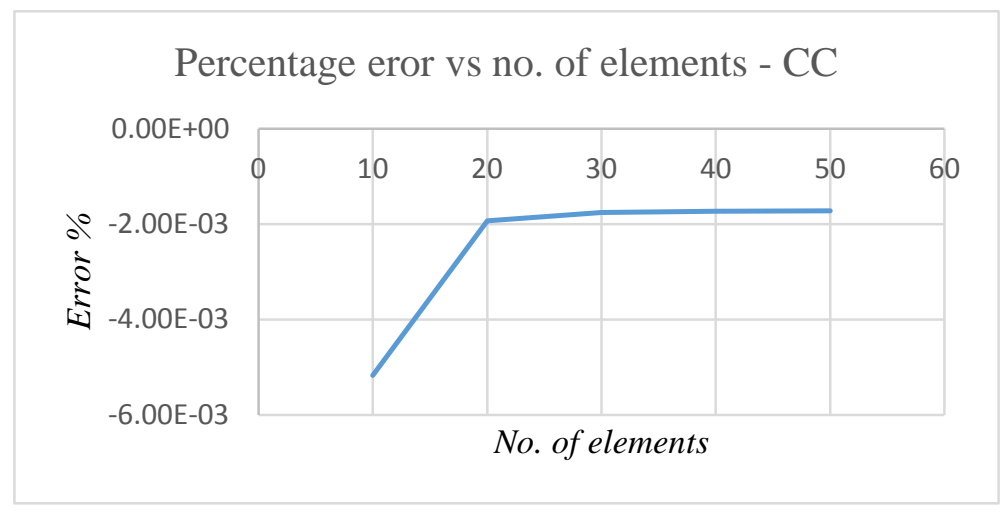

Figure 3.3 - Percentage error vs. the number of elements - CC - Up to 50 FEM elements 


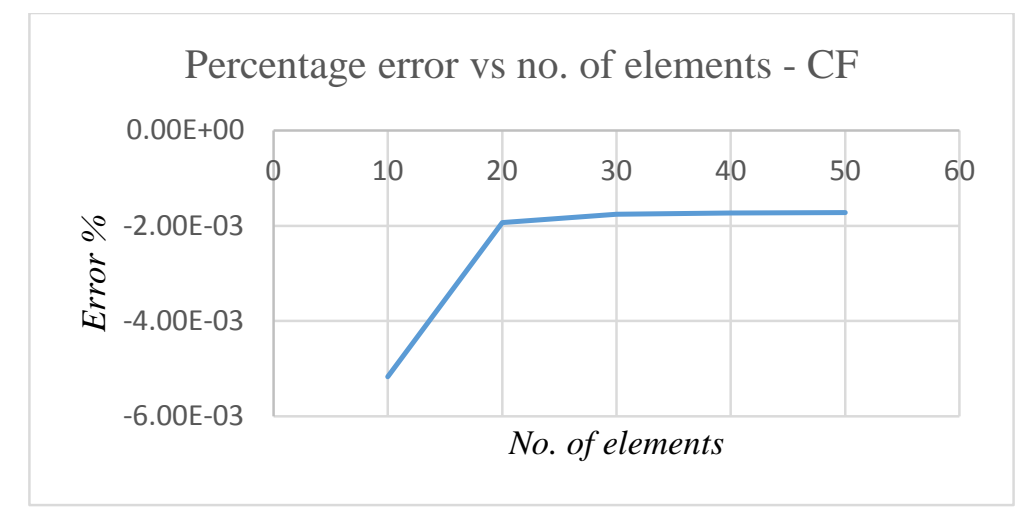

Figure 3.4 - Percentage error vs. the number of elements - CF - Up to 50 FEM elements

It can be noticed on the vertical axis of Figures (3.2) - (3.4) that percentage of error is in the order of $10^{-4}$ even for 10 elements which shows high convergence rates but the study considers 50 elements so as to more precise. High convergence rates, like these are found throughout the thesis.

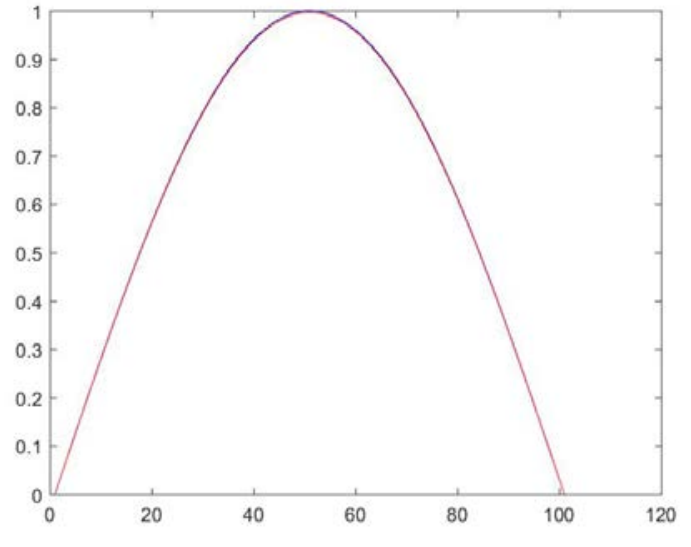

Mode 1

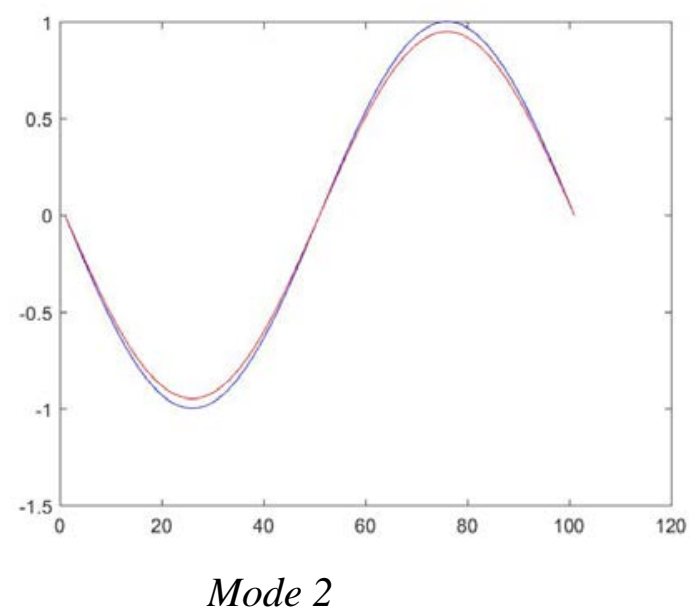

Mode 2 


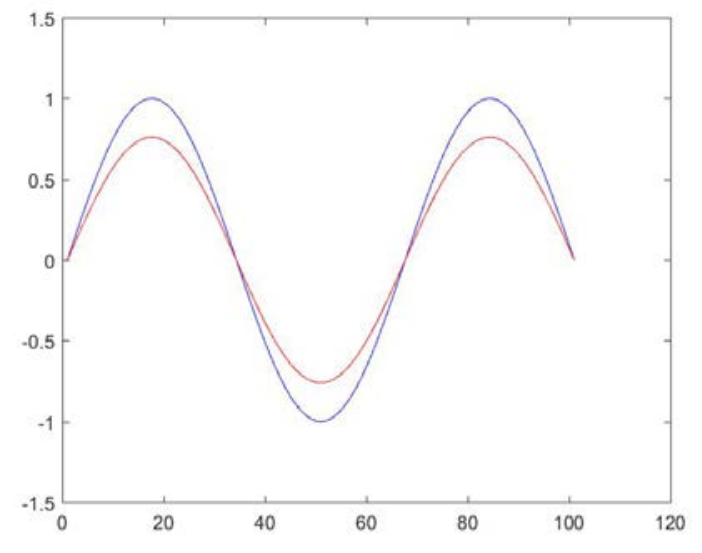

Mode 3

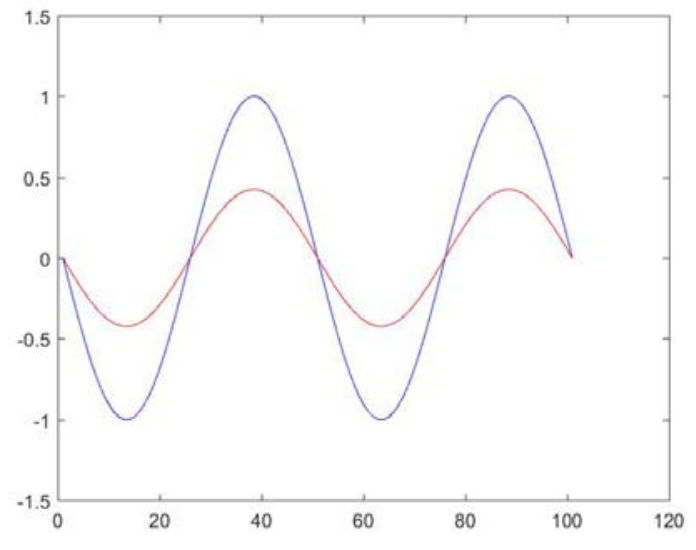

Mode 4

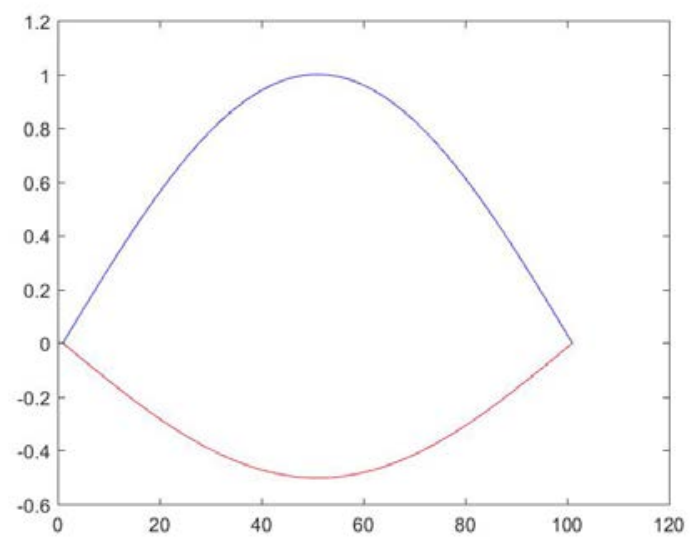

Mode 5

Figure 3.5 - First two mode shapes of DWCNT modeled as local Euler-Bernoulli beam - simply supported boundary condition - FEM

Figures (3.5) show the mode shapes of the DWCNT for simply supported boundary conditions. It can be seen that the first few mode shapes are coaxial and tend to be non-coaxial when mode number increases. It can be seen that as the mode shapes tend to be non-coaxial, the concentric nature of the multi-walled carbon nanotube is distorted. This is not possible physically as that would mean the inner tube tears the outer tube in order to vibrate. Though the governing equations of a continuum structure gives $n$ - number of mode shapes, not all of them might be physically possible. The resonant frequencies are governed by the amplitude ratio of inner tube to outer given by [195]: 


$$
f=1+\frac{E_{2} I_{2} \lambda_{1}^{4}}{c}-\frac{m_{2} \omega^{2}}{c}
$$

For the first frequency, $f$ is always close to unity and hence the vibrational modes are coaxial. As the value of $f$ varies in either direction, the vibrational modes tend to be non-coaxial.
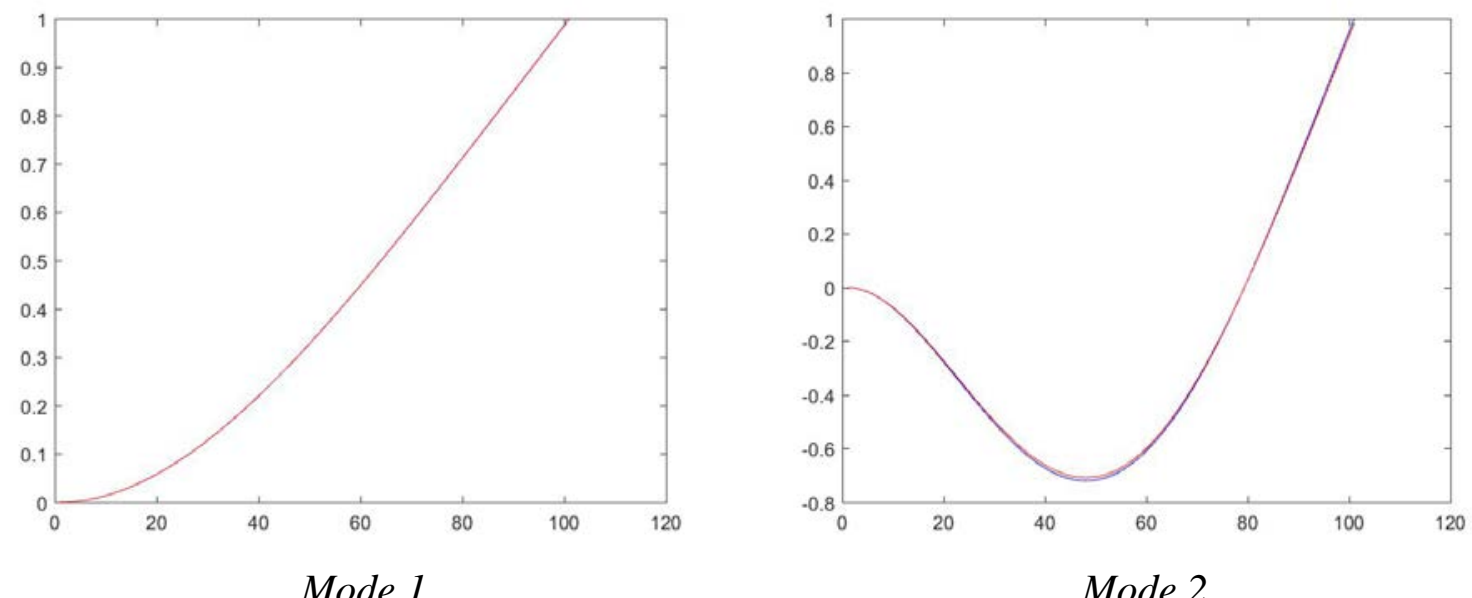

Figure 3.6 - First two mode shapes of DWCNT modeled as local Euler-Bernoulli beam clamped-free boundary condition - FEM

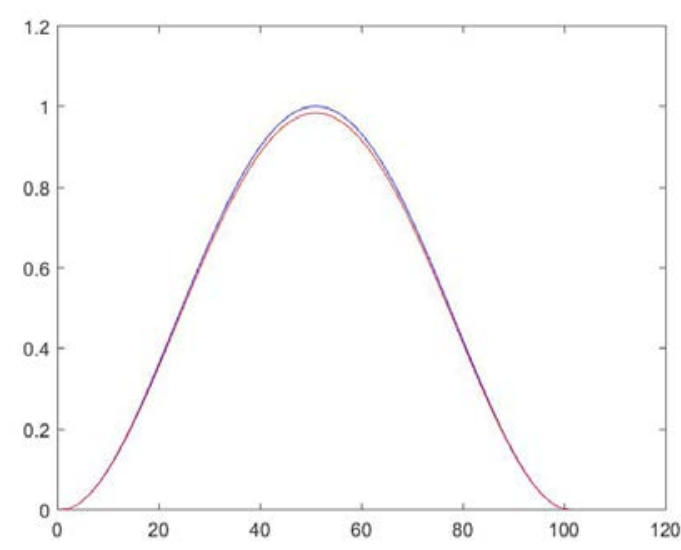

Mode 1

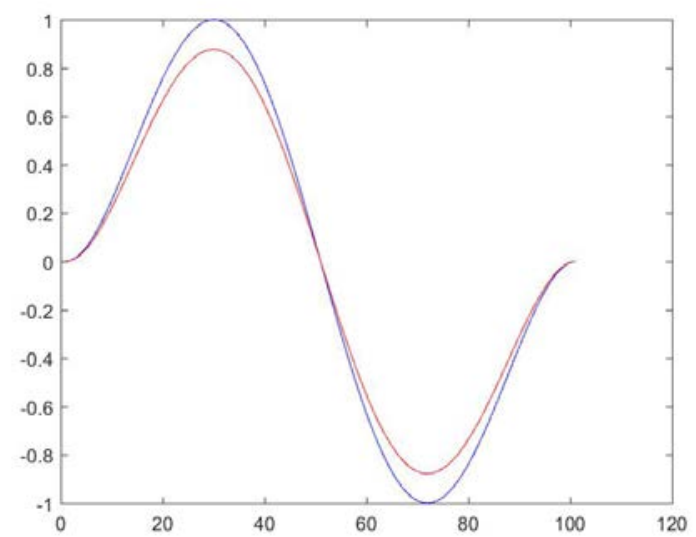

Mode 2

Figure 3.7 - First two mode shapes of DWCNT modeled as local Euler-Bernoulli beam clamped-clamped boundary condition - FEM 


\subsubsection{DWCNTs, modeled as local Euler-Bernoulli beam embedded in an elastic medium}

Composite structures have been spearheading the use of carbon nanotubes as fillers or fibers, dispersed uniformly or as clusters, to improve the properties of resulting structure. Hence, studying the behavior of carbon nanotubes embedded in an elastic medium gains importance. The natural frequencies of DWCNTs embedded in an elastic medium are shown in Table 3.4, where four cases are taken to represent the pressure exerted by the external medium on the CNT. The results are compared to the values given by Yoon et al. [41] to verify the accuracy and robustness of the present study. Yoon et al. [41] solved the governing equations for CNTs embedded in an elastic medium to obtain the natural frequencies.

The four values of the spring constant, $k$, are assumed such that the ratio $k / c$ is much higher, much lower, comparatively close to unity and at unity itself. The results show that there is a close agreement with the values calculated by Yoon et al. [41] and the percentage error remains small, as shown in the Table 3.3. Finite element results considers 50 elements, except for the case of clamped free boundary condition which uses 40 elements, for sake of convergence i.e., the number of elements are increased to the point that the percentage error between them becomes zero or becomes stable. Figures (3.8)-(3.10) show the percentage error vs. number of elements, where the values given by Yoon et al. [41], for ratio $k / c=0.0001$, are used as benchmark using which the percentage error is calculated.

It is observed from Table 3.3 that the stiffness of the material in which the carbon nanotube is embedded has an effect on the natural frequency of the carbon nanotube; i.e., if the elastic medium has the stiffness $k \gg 1$ say, $k / c=100$, the natural frequency of the carbon nanotube increases at least by one order for all the boundary conditions, as can be observed from Table 3.3. It can also be seen that as the stiffness of the elastic medium reduces, the natural frequency decreases due to the fact that carbon nanotubes would then have a less stiff material to vibrate in. These observations are meant for the outer tube of DWCNT, as the inner tube will have van der Waals forces around them, and so the coaxial frequency of DWCNT for the case when $k \ll 1$, i.e., say $k / c=0.0001$, remains comparable whether or not the carbon nanotube is embedded in the elastic matrix. 
Table 3.3 - FEM natural frequencies of DWCNTs modeled as local Euler-Bernoulli beam, embedded in an elastic medium

\begin{tabular}{|c|c|c|c|c|}
\hline \multirow[b]{2}{*}{$\begin{array}{l}\text { Boundary } \\
\text { Conditions }\end{array}$} & \multirow[b]{2}{*}{$\mathrm{k} / \mathrm{c}$ ratio } & \multirow[b]{2}{*}{$\begin{array}{l}\text { Exact Value [41] } \\
\qquad\left(10^{12} \mathrm{rad} / \mathrm{s}\right)\end{array}$} & \multicolumn{2}{|c|}{ Present Study } \\
\hline & & & $\begin{array}{c}\text { Natural } \\
\text { Frequencies } \\
\left(10^{12} \mathrm{rad} / \mathrm{s}\right)\end{array}$ & $\begin{array}{c}\text { Percentage } \\
\text { Error }\end{array}$ \\
\hline \multirow{8}{*}{ Simply Supported } & \multirow{2}{*}{$k / c=100$} & 6.404 & 6.404 & $0.00 \%$ \\
\hline & & 45.704 & 45.704 & $0.00 \%$ \\
\hline & \multirow{2}{*}{$k / c=1$} & 3.507 & 3.506 & $0.00 \%$ \\
\hline & & 8.413 & 8.412 & $0.00 \%$ \\
\hline & \multirow{2}{*}{$k / c=0.1$} & 1.256 & 1.256 & $0.00 \%$ \\
\hline & & 7.929 & 7.929 & $0.00 \%$ \\
\hline & \multirow{2}{*}{$k / c=0.0001$} & 0.468 & 0.468 & $0.00 \%$ \\
\hline & & 7.885 & 7.885 & $0.00 \%$ \\
\hline \multirow{8}{*}{ Clamped Clamped } & \multirow{2}{*}{$k / c=100$} & 6.398 & 6.431 & $0.50 \%$ \\
\hline & & 45.702 & 45.717 & $0.03 \%$ \\
\hline & \multirow{2}{*}{$k / c=1$} & 6.010 & 6.010 & $0.00 \%$ \\
\hline & & 10.101 & 10.100 & $0.00 \%$ \\
\hline & \multirow{2}{*}{$k / c=0.1$} & 1.570 & 1.570 & $0.00 \%$ \\
\hline & & 7.970 & 7.970 & $0.00 \%$ \\
\hline & \multirow{2}{*}{$k / c=0.0001$} & 1.058 & 1.058 & $0.00 \%$ \\
\hline & & 7.925 & 7.925 & $0.00 \%$ \\
\hline \multirow{8}{*}{ Clamped Free } & \multirow{2}{*}{$k / c=100$} & 6.398 & 6.398 & $0.00 \%$ \\
\hline & & 45.702 & 45.702 & $0.00 \%$ \\
\hline & \multirow{2}{*}{$k / c=1$} & 3.483 & 3.483 & $0.00 \%$ \\
\hline & & 8.403 & 8.403 & $0.00 \%$ \\
\hline & \multirow{2}{*}{$k / c=0.1$} & 1.179 & 1.179 & $0.00 \%$ \\
\hline & & 7.921 & 7.921 & $0.00 \%$ \\
\hline & \multirow{2}{*}{$k / c=0.0001$} & 0.170 & 0.170 & $0.01 \%$ \\
\hline & & 7.876 & 7.876 & $0.00 \%$ \\
\hline
\end{tabular}


The following figures show that the error percentage even for 10 elements is negligible i.e., in the order of $10^{-4}$ but more number of elements are considered for the sake of precision.

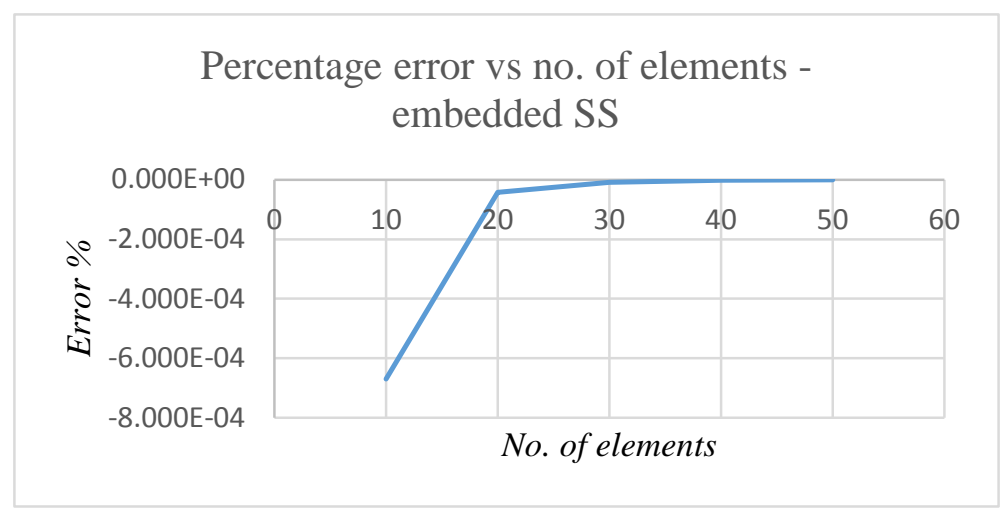

Figure 3.8 - Percentage error vs. the number of elements - embedded SS - Up to 50 FEM elements

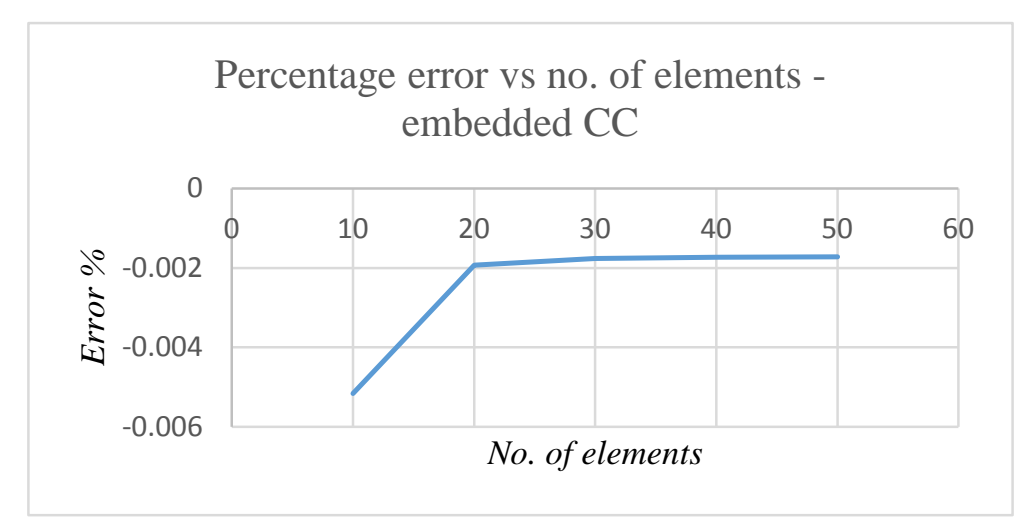

Figure 3.9 - Percentage error vs. the number of elements - embedded CC - Up to 50 FEM elements 


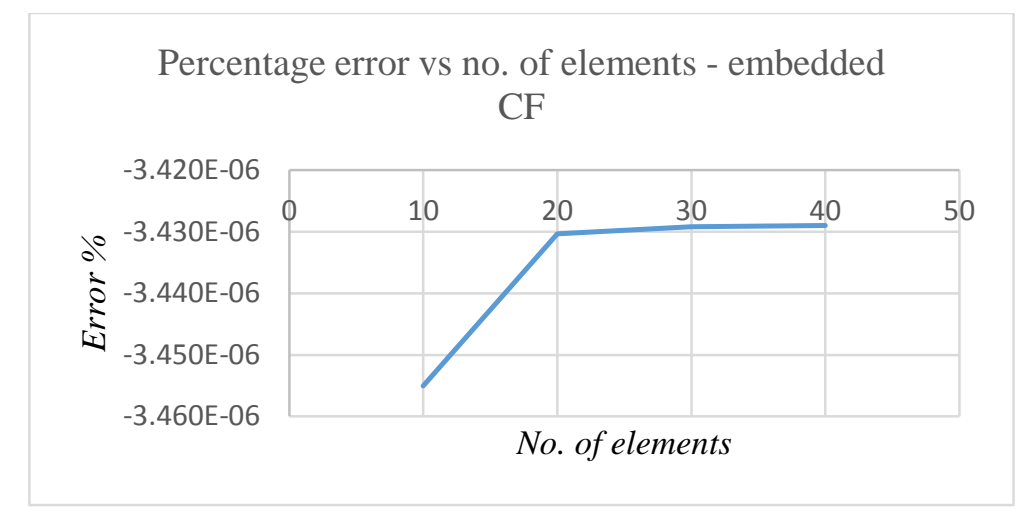

Figure 3.10 - Percentage error vs. the number of elements - embedded CF - Up to 40 FEM elements

The mode shapes for local Euler-Bernoulli beam based DWCNT, embedded in an elastic medium are presented in Figures (3.11) - (3.13) for the case of $k / c=0.1$. The mode shapes for other values of $k / c$ are presented in Appendix A3.

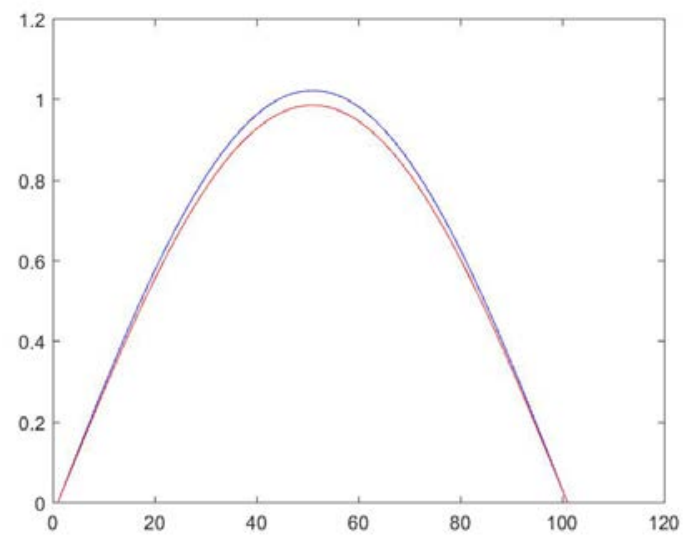

Mode 1

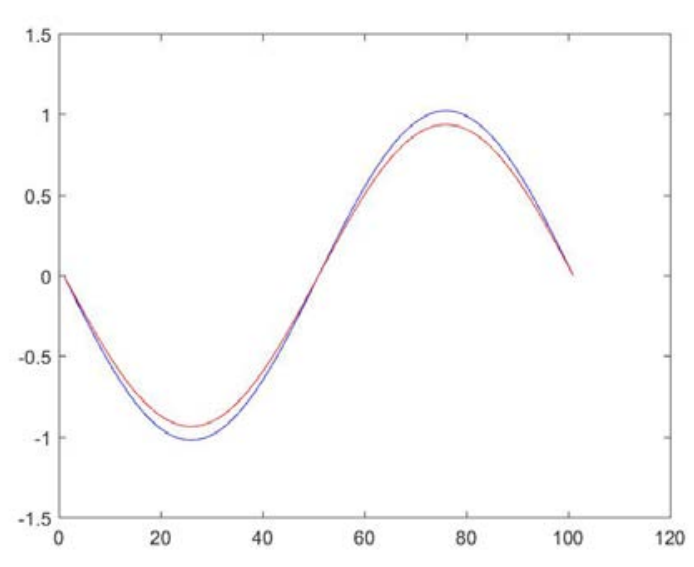

Mode 2

Figure 3.11 - First two mode shapes of DWCNT, modeled as local Euler-Bernoulli beam, embedded in elastic medium $(k / c=0.1)$ - simply supported boundary condition - FEM 


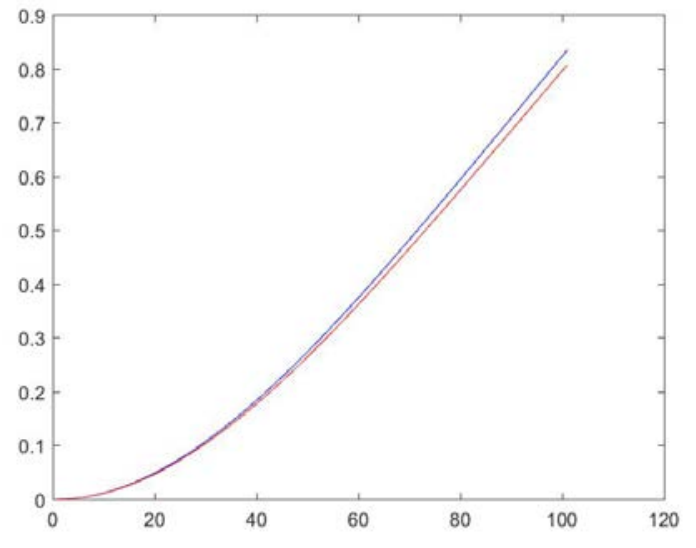

Mode 1

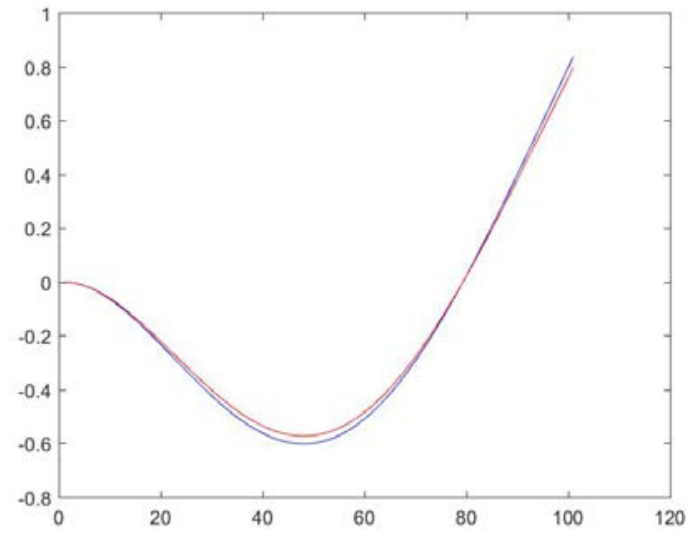

Mode 2

Figure 3.12 - First two mode shapes of DWCNT, modeled as local Euler-Bernoulli beam, embedded in elastic medium $(k / c=0.1)$ - clamped-free boundary condition - FEM

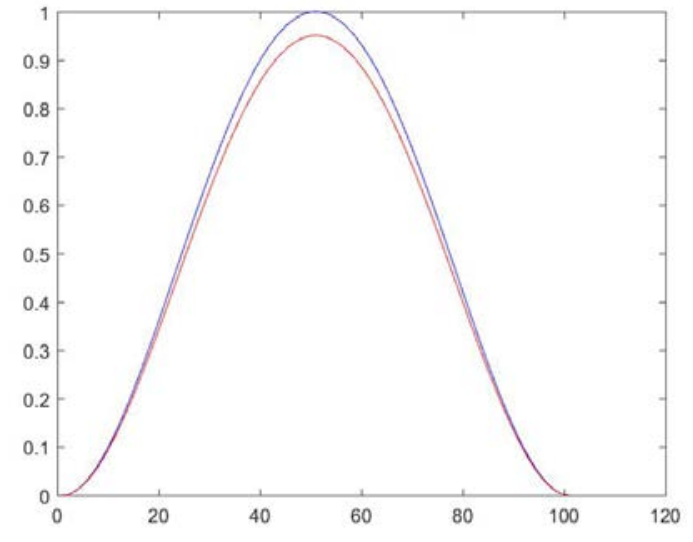

Mode 1

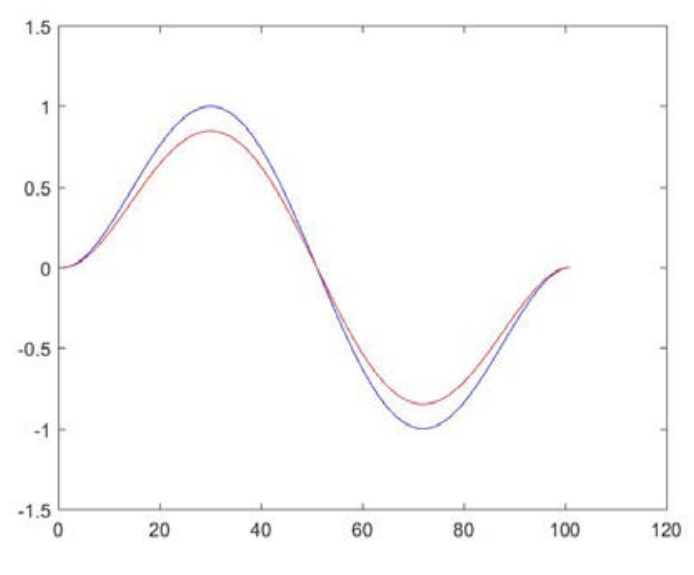

Mode 2

Figure 3.13 - First two mode shapes of DWCNT, modeled as local Euler-Bernoulli beam, embedded in elastic medium $(k / c=0.1)$ - clamped-clamped boundary condition - FEM

From Figures (3.11) to (3.13), it can be observed that as the mode number increases, vibration of DWCNT tends to be more non-coaxial than coaxial. The modes of DWCNT embedded in an elastic medium are governed by [41]:

$$
\frac{a_{1}}{a_{2}}=1+\frac{E_{2} I_{2} \lambda_{n}^{4}}{c}-\frac{\rho \omega^{2} A_{2}}{c}+\frac{k}{c}
$$


By comparing Figures (3.5) - (3.7) and Figures (3.11) - (3.13), it can be seen that mode shape of the DWCNT is not affected by it being embedded or not being embedded in an elastic medium.

\subsection{Conclusion}

In this chapter, based on local Euler-Bernoulli beam, a finite element model was formulated for two cases of a double-walled carbon nanotube, embedded and not embedded in elastic medium. The natural frequencies obtained using this formulation are in excellent agreement with the socalled exact and other values reported in literature. 


\section{Dynamic Stiffness Matrix Formulation: Double-Walled Carbon Nanotubes}

\subsection{Introduction}

Dynamic stiffness matrix (DSM) is formulated as a single, frequency-dependant, stiffness matrix by transforming the coupled governing equations as a single equation and finding a closed form solution for it. In this chapter, a DSM is formulated for double-walled carbon nanotubes, with and without surrounding elastic medium.

\subsection{Formulation}

The dynamic stiffness matrix for double-walled carbon nanotubes (DWCNTs) is derived using the same equations presented in Chapter 3 for finite element formulation (FEM), i.e., equations (3.5) and (3.6), duplicated here:

$$
\begin{aligned}
& E_{1} I_{1} w_{1}^{\prime \prime \prime \prime}+\rho_{1} A_{1} \ddot{w}_{1}=c w_{2}-c w_{1} \\
& E_{2} I_{2} w_{2}^{\prime \prime \prime \prime}+\rho_{2} A_{2} \ddot{w}_{2}=-\hat{c} w_{2}-c w_{1}
\end{aligned}
$$

Considering simple harmonic motion and using the transformations (3.9) and (3.10), and eliminating the time dependency, the following equations are obtained:

$$
\begin{array}{r}
E_{1} I_{1} W_{1}^{\prime \prime \prime}-m_{1} \omega^{2} W_{1}+c W_{1}-c W_{2}=0 \\
E_{2} I_{2} W_{2}^{\prime \prime \prime \prime}-m_{2} \omega^{2} W_{2}+\widehat{c} W_{2}-c W_{1}=0
\end{array}
$$

The $\hat{c}$ term in (4.4) follows the rule set using (3.7) and (3.8), i.e., $\hat{c}$ would mean $\hat{c}$ for the case of a DWCNT without elastic medium and would represent $(c+k)$ when the nanotubes are embedded in an elastic medium. Rewriting the above equations by introducing a differential operator, $D=d / d x$ leads to:

$$
\left(E_{1} I_{1} D^{4}-m_{1} \omega^{2}+c\right) W_{1}-c W_{2}=0
$$




$$
\left(E_{2} I_{2} D^{4}-m_{2} \omega^{2}+\widehat{c}\right) W_{2}-c W_{1}=0
$$

Equations (4.5) and (4.6) are now combined to obtain an eighth order equation in terms of either $W_{1}$ or $W_{2}$, written as:

$$
\left(D^{8}+2 p D^{4}+q\right) Y=0
$$

where $Y$ is either $W_{1}$ or $W_{2}$ and

$$
\begin{gathered}
p=\frac{1}{2}\left(\frac{c}{E_{1} I_{1}}+\frac{c}{E_{2} I_{2}}\right)-\frac{\omega^{2}}{2}\left(\frac{m_{1}}{E_{1} I_{1}}+\frac{m_{2}}{E_{2} I_{2}}\right) \\
q=\frac{m_{1} m_{2} \omega^{4}-c w^{2}\left(m_{1}+m_{2}\right)-c^{2}+c \widehat{C}}{\left(E_{1} I_{1}\right)\left(E_{2} I_{2}\right)}
\end{gathered}
$$

It is worth noting that (4.9) reduces to (4.9.1), when $\widehat{c}=c$, i.e., carbon nanotubes not embedded in an elastic medium.

$$
q=\frac{m_{1} m_{2} \omega^{4}-c w^{2}\left(m_{1}+m_{2}\right)}{\left(E_{1} I_{1}\right)\left(E_{2} I_{2}\right)}
$$

The solution of equation (4.7) is sought in the form of

$$
w_{j}=Y_{j}(x) e^{i \kappa t}
$$

where $j=1,2$. Equation (4.7) is now written as:

$$
\left(\kappa^{2}+2 p \kappa+q\right) Y=0
$$

with $\kappa=D^{4}$. This reduces the eighth order equation to a quadratic one, the roots of which are $\alpha$ and $\beta$, defined below for two cases;

Case 1: When $q<0$ :

$$
\begin{gathered}
\alpha=-p+\sqrt{p^{2}-q} \\
\beta=p+\sqrt{p^{2}-q}
\end{gathered}
$$

Case 2: When $q>0$ : 


$$
\begin{aligned}
& \alpha=-p+\sqrt{p^{2}-q} \\
& \beta=-p-\sqrt{p^{2}-q}
\end{aligned}
$$

The eight roots of the polynomial equation are 4 real and 4 imaginary roots, written as:

$$
\alpha,-\alpha, i \alpha,-i \alpha ; \beta,-\beta, i \beta,-i \beta
$$

It is worth mentioning that in both cases of DWCNTs (i.e., with and without the surrounding elastic medium), the roots and hence the solution remains the same. The solution of the governing equation is then expressed as:

$$
Y=D_{1} e^{\sqrt[4]{\alpha} x}+D_{2} e^{-\sqrt[4]{\alpha} x}+D_{3} \cos (\sqrt[4]{\alpha} x)+D_{4} \sin (\sqrt[4]{\alpha} x)+D_{5} e^{\sqrt[4]{\beta} x}+D_{6} e^{-\sqrt[4]{\beta} x}+D_{7} \cos (\sqrt[4]{\beta} x)+D_{8} \sin (\sqrt[4]{\beta} x)
$$

where $Y$ is either $W_{1}$ or $W_{2}$, and $D_{1-8}$ are arbitrary constants. Rewriting the solutions as:

$$
\begin{aligned}
& W_{1}=A_{1} e^{\sqrt[4]{\alpha} x}+A_{2} e^{-\sqrt[4]{\alpha} x}+A_{3} \cos (\sqrt[4]{\alpha} x)+A_{4} \sin (\sqrt[4]{\alpha} x)+A_{5} e^{\sqrt[4]{\beta} x}+A_{6} e^{-\sqrt[4]{\beta} x}+A_{7} \cos (\sqrt[4]{\beta} x)+A_{8} \sin (\sqrt[4]{\beta} x) \\
& W_{2}=B_{1} e^{\sqrt[4]{\alpha} x}+B_{2} e^{-\sqrt[4]{\alpha} x}+B_{3} \cos (\sqrt[4]{\alpha} x)+B_{4} \sin (\sqrt[4]{\alpha} x)+B_{5} e^{\sqrt[4]{\beta} x}+B_{6} e^{-\sqrt[4]{\beta} x}+B_{7} \cos (\sqrt[4]{\beta} x)+B_{8} \sin (\sqrt[4]{\beta} x)
\end{aligned}
$$

where $A_{1-8}$ or $B_{1-8}$ are two sets of constants and are related to each other through the following expressions, obtained by substituting (4.16) and (4.17) in either (4.3) or (4.4):

$$
\begin{array}{rlll}
B_{1}=\lambda_{1} A_{1} & B_{2}=\lambda_{1} A_{2} & B_{3}=\lambda_{1} A_{3} & B_{4}=\lambda_{1} A_{4} \\
B_{5}=\lambda_{2} A_{5} & B_{6}=\lambda_{2} A_{6} & B_{7}=\lambda_{2} A_{7} & B_{8}=\lambda_{2} A_{8}
\end{array}
$$

where $\lambda_{1}$ and $\lambda_{2}$ take different values for each case, as given below:

$$
\lambda_{1}=1+\frac{E_{1} I_{1} \alpha}{c}-\frac{m_{1} \omega^{2}}{c} \text { and } \lambda_{2}=1+\frac{E_{1} I_{1}(-\beta)}{c}-\frac{m_{1} \omega^{2}}{c}
$$

for case 1 and 


$$
\lambda_{1}=1+\frac{E_{1} I_{1} \alpha}{c}-\frac{m_{1} \omega^{2}}{c} \text { and } \lambda_{2}=1+\frac{E_{1} I_{1} \beta}{c}-\frac{m_{1} \omega^{2}}{c}
$$

for case 2. The modified equations (4.16) and (4.17) are re-written as:

$W_{1}=A_{1} e^{\sqrt[4]{\alpha} x}+A_{2} e^{-\sqrt[4]{\alpha} x}+A_{3} \cos (\sqrt[4]{\alpha} x)+A_{4} \sin (\sqrt[4]{\alpha} x)+A_{5} e^{\sqrt[4]{\beta} x}+A_{6} e^{-\sqrt[4]{\beta} x}+A_{7} \cos (\sqrt[4]{\beta} x)+A_{8} \sin (\sqrt[4]{\beta} x)$

$W_{2}=A_{1} \lambda_{1} e^{\sqrt[4]{\alpha} x}+A_{2} \lambda_{1} e^{-\sqrt[4]{\alpha} x}+A_{3} \lambda_{1} \cos (\sqrt[4]{\alpha} x)+A_{4} \lambda_{1} \sin (\sqrt[4]{\alpha} x)+A_{5} \lambda_{2} e^{\sqrt[4]{\beta} x}+A_{6} \lambda_{2} e^{-\sqrt[4]{\beta} x}+A_{7} \lambda_{2} \cos (\sqrt[4]{\beta} x)$

$+A_{8} \lambda_{2} \sin (\sqrt[4]{\beta} x)$

The slopes (bending rotations of $W_{1}$ and $W_{2}$.) for each of the nanotubes are then written as:

$\theta_{1}=\frac{d W_{1}}{d x}$

$=A_{1} \alpha^{1 / 4} e^{\alpha^{1 / 4} x}-A_{2} \alpha^{1 / 4} e^{-\alpha^{1 / 4} x}-A_{3} \alpha^{1 / 4} \sin \left(\alpha^{1 / 4} x\right)+A_{4} \alpha^{1 / 4} \cos \left(\alpha^{1 / 4} x\right)+A_{5} \beta^{1 / 4} e^{\alpha^{1 / 4} x}-A_{6} \beta^{1 / 4} e^{\beta^{1 / 4} x}$

$-A_{7} \beta^{1 / 4} \cos \left(\beta^{1 / 4} x\right)+A_{8} \beta^{1 / 4} \sin \left(\beta^{1 / 4} x\right)$

$\theta_{2}=\frac{d W_{2}}{d x}$

$=A_{1} \lambda_{1} \alpha^{1 / 4} e^{\alpha^{1 / 4} x}-A_{2} \lambda_{1} \alpha^{1 / 4} e^{-\alpha^{1 / 4} x}-A_{3} \lambda_{1} \alpha^{1 / 4} \sin \left(\alpha^{1 / 4} x\right)+A_{4} \lambda_{1} \alpha^{1 / 4} \cos \left(\alpha^{1 / 4} x\right)+A_{5} \lambda_{2} \beta^{1 / 4} e^{\alpha^{1 / 4} x}$

$-A_{6} \lambda_{2} \beta^{1 / 4} e^{\beta^{1 / 4 x}}-A_{7} \lambda_{2} \beta^{1 / 4} \cos \left(\beta^{1 / 4} x\right)+A_{8} \lambda_{2} \beta^{1 / 4} \sin \left(\beta^{1 / 4} x\right)$

The expressions for bending moments (4.23) are written as:

$$
\begin{aligned}
& M_{1}=-E_{1} I_{1} \frac{d^{2} W_{1}}{d x^{2}} \\
& =-E_{1} I_{1}\left(A_{1} \sqrt{\alpha} e^{\alpha^{1 / 4 x}}+A_{2} \sqrt{\alpha} e^{-\alpha^{1 / 4 x}}-A_{3} \sqrt{\alpha} \cos \left(\alpha^{1 / 4} x\right)+A_{4} \sqrt{\alpha} \sin \left(\alpha^{1 / 4} x\right)+A_{5} \sqrt{\beta} e^{\beta^{1 / 4 x}}+A_{6} \sqrt{\beta} e^{-\beta^{1 / 4} x}\right. \\
& \left.-A_{7} \sqrt{\beta} \cos \left(\beta^{1 / 4} x\right)-A_{8} \sqrt{\beta} \sin \left(\beta^{1 / 4} x\right)\right) \\
& M_{2}=-E_{2} I_{2} \frac{d^{2} W_{2}}{d x^{2}}
\end{aligned}
$$




$$
\begin{aligned}
& =-E_{2} I_{2}\left(A_{1} \lambda_{1} \sqrt{\alpha} e^{\alpha^{1 / 4} x}+A_{2} \lambda_{1} \sqrt{\alpha} e^{-\alpha^{1 / 4} x}-A_{3} \lambda_{1} \sqrt{\alpha} \cos \left(\alpha^{1 / 4} x\right)+A_{4} \lambda_{1} \sqrt{\alpha} \sin \left(\alpha^{1 / 4} x\right)+A_{5} \lambda_{2} \sqrt{\beta} e^{\beta^{1 / 4 x}}\right. \\
& \left.+A_{6} \lambda_{2} \sqrt{\beta} e^{-\beta^{1 / 4} x}-A_{7} \lambda_{2} \sqrt{\beta} \cos \left(\beta^{1 / 4} x\right)-A_{8} \lambda_{2} \sqrt{\beta} \sin \left(\beta^{1 / 4} x\right)\right)
\end{aligned}
$$

The following expressions (4.24) represent the resulting (internal) shear forces for $W_{1}$ and $W_{2}$,

$$
\begin{aligned}
& S_{1}=E_{1} I_{1} \frac{d^{3} W_{1}}{d x^{3}} \\
& =E_{1} I_{1}\left(A_{1} \alpha^{3 / 4} e^{\alpha^{1 / 4} x}-A_{2} \alpha^{3 / 4} e^{-\alpha^{1 / 4} x}+A_{3} \alpha^{3 / 4} \sin \left(\alpha^{1 / 4} x\right)-A_{4} \alpha^{3 / 4} \cos \left(\alpha^{1 / 4} x\right)+A_{5} \beta^{3 / 4} e^{\beta^{1 / 4}}-A_{6} \beta^{3 / 4} e^{-\beta^{1 / 4} x}\right. \\
& +A_{7} \beta^{3 / 4} \sin \left(\beta^{1 / 4} x\right)-A_{8} \beta^{3 / 4} \cos \left(\beta^{1 / 4} x\right) \\
& S_{2}=E_{2} I_{2} \frac{d^{3} W_{2}}{d x^{3}} \\
& =E_{2} I_{2}\left(A_{1} \lambda_{1} \alpha^{3 / 4} e^{\alpha^{1 / 4} x}-A_{2} \lambda_{1} \alpha^{3 / 4} e^{-\alpha^{1 / 4} x}+A_{3} \lambda_{1} \alpha^{3 / 4} \sin \left(\alpha^{1 / 4} x\right)-A_{4} \lambda_{1} \alpha^{3 / 4} \cos \left(\alpha^{1 / 4} x\right)+A_{5} \lambda_{2} \beta^{3 / 4} e^{\beta^{1 / 4} x}\right. \\
& -A_{6} \lambda_{2} \beta^{3 / 4} e^{-\beta^{1 / 4} x}+A_{7} \lambda_{2} \beta^{3 / 4} \sin \left(\beta^{1 / 4} x\right)-A_{8} \lambda_{2} \beta^{3 / 4} \cos \left(\beta^{1 / 4} x\right)
\end{aligned}
$$

These expressions are now used to generate the dynamic stiffness matrix (DSM), by enforcing end conditions to both the displacements, (4.21) and (4.22), and loads, (4.23) and (4.24).

For displacements:

At $x=0$ :

$Y_{1}=W_{1} ; Y_{2}=W_{2} ; T_{1}=\theta_{1} ; T_{2}=\theta_{2}$

At $x=L$ :

$Y_{3}=W_{1} ; Y_{4}=W_{2} ; T_{3}=\theta_{1} ; T_{4}=\theta_{2}$ 
For shear forces and bending moments:

At $x=0$ :

$S_{1}=S_{1} ; S_{2}=S_{2} ; M_{1}=M_{1} ; M_{2}=M_{2}$

At $x=L$ :

$S_{3}=-S_{1} ; S_{4}=-S_{2} ; M_{3}=-M_{1} ; M_{4}=-M_{2}$

Using (4.25) in (4.21) and (4.22), the following expressions are obtained:

$$
\delta=H A
$$

where

$$
\delta=\left[\begin{array}{llllllll}
Y_{1} & Y_{2} & T_{1} & T_{2} & Y_{3} & Y_{4} & T_{3} & T_{4}
\end{array}\right]^{T}, A=\left[\begin{array}{llllllll}
A_{1} & A_{2} & A_{3} & A_{4} & A_{5} & A_{6} & A_{7} & A_{8}
\end{array}\right]^{T} ;
$$

$$
H=\left[\begin{array}{cccccccc}
1 & 1 & 1 & 0 & 1 & 1 & 1 & 0 \\
\lambda_{1} & \lambda_{1} & \lambda_{1} & 0 & \lambda_{2} & \lambda_{2} & \lambda_{2} & 0 \\
\alpha^{1 / 4} & -\alpha^{1 / 4} & 0 & \alpha^{1 / 4} & \beta^{1 / 4} & -\beta^{1 / 4} & 0 & \beta^{1 / 4} \\
\lambda_{1} \alpha^{1 / 4} & -\lambda_{1} \alpha^{1 / 4} & 0 & \lambda_{1} \alpha^{1 / 4} & \lambda_{2} \beta^{1 / 4} & -\lambda_{2} \beta^{1 / 4} & 0 & \lambda_{2} \beta^{1 / 4} \\
e^{\alpha^{1 / 4}} & e^{-\alpha^{1 / 4}} & C_{\alpha} & S_{\alpha} & e^{\beta^{1 / 4}} & e^{-\beta^{1 / 4}} & C_{\beta} & S_{\beta} \\
\lambda_{1} e^{\alpha^{1 / 4}} & \lambda_{1} e^{-\alpha^{1 / 4}} & \lambda_{1} C_{\alpha} & \lambda_{1} S_{\alpha} & \lambda_{2} e^{\beta^{1 / 4}} & \lambda_{2} e^{-\beta^{1 / 4}} & \lambda_{2} C_{\beta} & \lambda_{2} S_{\beta} \\
\alpha^{1 / 4} e^{\alpha^{1 / 4}} & -\alpha^{1 / 4} e^{-\alpha^{1 / 4}} & -\alpha^{1 / 4} S_{\alpha} & \alpha^{1 / 4} C_{\alpha} & \beta^{1 / 4} e^{\beta^{1 / 4}} & -\beta^{1 / 4} e^{-\beta^{1 / 4}} & -\beta^{1 / 4} S_{\beta} & \beta^{1 / 4} C_{\beta} \\
\lambda_{1} \alpha^{1 / 4} e^{\alpha^{1 / 4}} & -\lambda_{1} \alpha^{1 / 4} e^{-\alpha^{1 / 4}} & -\lambda_{1} \alpha^{1 / 4} S_{\alpha} & \lambda_{1} \alpha^{1 / 4} C_{\alpha} & \lambda_{2} \beta^{1 / 4} e^{\beta^{1 / 4}} & -\lambda_{2} \beta^{1 / 4} e^{-\beta^{1 / 4}} & -\lambda_{2} \beta^{1 / 4} S_{\beta} & \lambda_{2} \beta^{1 / 4} C_{\beta}
\end{array}\right]
$$

where

$$
S_{\alpha}=\sin \left(\alpha^{1 / 4}\right) \quad C_{\alpha}=\cos \left(\alpha^{1 / 4}\right) \quad S_{\beta}=\sin \left(\beta^{1 / 4}\right) \quad C_{\beta}=\cos \left(\beta^{1 / 4}\right)
$$

In a similar fashion, (4.25) is used in (4.23) and (4.24), to obtain:

$$
F=Q A
$$

with 


$$
F=\left[\begin{array}{llllllll}
S_{1} & S_{2} & M_{1} & M_{2} & S_{3} & S_{4} & M_{3} & M_{4}
\end{array}\right]^{T},
$$

and $A$ already defined in (4.28) and

$$
Q=\left[\begin{array}{cccccccc}
\tau_{1} \varepsilon_{1} & -\tau_{1} \varepsilon_{1} & 0 & -\tau_{1} \varepsilon_{1} & \tau_{1} \varepsilon_{2} & -\tau_{1} \varepsilon_{2} & 0 & -\tau_{1} \varepsilon_{2} \\
\tau_{2} \lambda_{1} \varepsilon_{1} & -\tau_{2} \lambda_{1} \varepsilon_{1} & 0 & -\tau_{2} \lambda_{1} \varepsilon_{1} & \tau_{2} \lambda_{2} \varepsilon_{2} & -\tau_{2} \lambda_{2} \varepsilon_{2} & 0 & -\tau_{2} \lambda_{2} \varepsilon_{2} \\
-\tau_{1} \eta_{1} & -\tau_{1} \eta_{1} & \tau_{1} \eta_{1} & 0 & -\tau_{1} \eta_{2} & -\tau_{1} \eta_{2} & \tau_{1} \eta_{2} & 0 \\
-\tau_{2} \lambda_{1} \eta_{1} & -\tau_{2} \lambda_{1} \eta_{1} & \tau_{2} \lambda_{1} \eta_{1} & 0 & -\tau_{2} \lambda_{2} \eta_{2} & -\tau_{2} \lambda_{2} \eta_{2} & \tau_{2} \lambda_{2} \eta_{2} & 0 \\
-\tau_{1} \varepsilon_{1} e^{\alpha^{1 / 4}} & \tau_{1} \varepsilon_{1} e^{-\alpha^{1 / 4}} & -\tau_{1} \varepsilon_{1} S_{\alpha} & \tau_{1} \varepsilon_{1} C_{\alpha} & -\tau_{1} \varepsilon_{2} e^{\beta^{1 / 4}} & \tau_{1} \varepsilon_{2} e^{-\beta^{1 / 4}} & -\tau_{1} \varepsilon_{2} S_{\beta} & \tau_{1} \varepsilon_{2} C_{\beta} \\
-\tau_{2} \varepsilon_{1} e^{\alpha^{1 / 4}} & \tau_{2} \varepsilon_{1} e^{-\alpha^{1 / 4}} & -\tau_{2} \varepsilon_{1} S_{\alpha} & \tau_{2} \varepsilon_{1} C_{\alpha} & -\tau_{2} \varepsilon_{2} e^{\beta^{1 / 4}} & \tau_{2} \varepsilon_{2} e^{-\beta^{1 / 4}} & -\tau_{2} \varepsilon_{2} S_{\beta} & \tau_{2} \varepsilon_{2} C_{\beta} \\
\tau_{1} \eta_{1} e^{\alpha^{1 / 4}} & \tau_{1} \eta_{1} e^{-\alpha / 4} & -\tau_{1} \eta_{1} C_{\alpha} & -\tau_{1} \eta_{1} S_{\alpha} & \tau_{1} \eta_{2} e^{\beta^{1 / 4}} & \tau_{1} \eta_{2} e^{-\beta^{1 / 4}} & -\tau_{1} \eta_{2} C_{\beta} & -\tau_{1} \eta_{2} S_{\beta} \\
\tau_{2} \lambda_{1} \eta_{1} e^{\alpha^{1 / 4}} & \tau_{2} \lambda_{1} \eta_{1} e^{-\alpha^{1 / 4}} & -\tau_{2} \lambda_{1} \eta_{1} C_{\alpha} & -\tau_{2} \lambda_{1} \eta_{1} S_{\alpha} & \tau_{2} \lambda_{2} \eta_{2} e^{\beta^{1 / 4}} & \tau_{2} \lambda_{2} \eta_{2} e^{-\beta^{1 / 4}} & -\tau_{2} \lambda_{2} \eta_{2} C_{\beta} & -\tau_{2} \lambda_{2} \eta_{2} S_{\beta}
\end{array}\right]
$$

where $S_{\alpha}, C_{\alpha}, S_{\beta}$, and $C_{\beta}$ are used as defined in (4.30) and

$$
\tau_{1}=E_{1} I_{1} \quad \tau_{2}=E_{2} I_{2} \quad \eta_{1}=\sqrt{\alpha} \quad \eta_{2}=\sqrt{\beta} \quad \varepsilon_{1}=\alpha^{3 / 4} \quad \varepsilon_{2}=\beta^{3 / 4}
$$

In (4.28) and (4.32), the column vectors are shown in transposed form for the sake of brevity. The system's dynamic stiffness matrix (DSM) is then obtained by relating the loads $F$ with displacements $\delta$, as represented in matrix form in (4.35)

$$
F=K \delta
$$

where

$$
K=Q H^{-1}
$$

is the required frequency-dependent, dynamic stiffness matrix for a system of double-walled carbon nanotubes (DWCNT), presented for the first time in this thesis. 


\subsection{Wittrick-Williams Method}

The developed dynamic stiffness matrix (DSM) can now be used to find the natural frequencies of the DWCNTs modeled as local Euler-Bernoulli beam by solving the system's nonlinear eigenvalue problem written as:

$$
[K(\omega)]\left\{w_{n}\right\}=0
$$

where $[K(\omega)]$ is dynamic stiffness matrix derived earlier. It is to be noticed that the dynamic stiffness matrix is different from finite element method matrices, in the sense that the DSM represents both the stiffness and mass properties of the system embedded in a single frequencydependent one, and hence results in a nonlinear eigenvalue problem. The use of a frequencydependent stiffness matrix ensures that none of the natural frequencies are missed. One of the most frequently used method to determine the natural frequencies, in this case, is the WittrickWilliams (W-W) algorithm [87,197], which uses a trial frequency and is based on Sturm sequence properties of a frequency-dependant stiffness matrix, is briefly explained here for completeness.

The number of frequencies exceeded by the trial frequency is given by

$$
J=J_{0}+\operatorname{sgn}\{K\}
$$

where $J_{0}$ represents the number of clamped-clamped natural frequency of all elements exceeded by the trial frequency and is given by

$$
J_{0}=\sum J_{m}
$$

where

$$
J_{m}=i-\frac{1}{2}\left[1-(-1)^{i} \operatorname{sg}\{D\}\right]
$$

and 
$i=\operatorname{int}\left(\frac{\omega^{*}}{\pi}\right) ; \operatorname{sg}\{D\}=1$ or $s g\{D\}=0$, depending on sign of $D$; i.e., $\operatorname{sg}\{D\}=1$, when $D$ is positive and negative when $D$ is negative. $D$ is defined as:

$$
D=1-\cosh \lambda \cos \lambda
$$

The sgn $\{K\}$ part in (4.38) represents the number of negative elements on the main diagonal of $K^{\Delta}$; i.e., $K(\omega)$ after upper triangulation is performed using Gauss elimination. It is to be noted that no row interchanges are to be performed in Gauss elimination. Bisection method is used to speed up the convergence of natural frequencies.

\subsection{Results and Discussion}

The 'exact' natural frequencies of DWCNTs modeled based on local Euler-Bernoulli beams are given below. The initial values are taken as given in equation (3.29)

\subsubsection{DWCNT modeled as local Euler-Bernoulli beams}

The natural frequencies of DWCNTs, modeled based on local Euler-Bernoulli beam, are presented in Table 4.1 and are compared with the exact values by $\mathrm{Xu}$ et al. [195] and with those obtained using FEM developed and reported earlier in Chapter 3 of this thesis document. It can be seen that the natural frequencies obtained using this method are in good agreement with the other methods. It is worth stating that DSM method is independent of number of elements and the resulting natural frequency is comparable to the natural frequency obtained using a 50element FEM model, hence justifying itself as 'exact' method. It is to be noted that the 'Error 1' in Tables 4.1 gives the error percentage with respect to the exact values given by Xu et al. [195] and 'Error 2' is calculated with respect to the natural frequency obtained using finite element formulation. 
Table 4.1 - DSM natural frequencies of DWCNTs, modeled as local Euler-Bernoulli beams

\begin{tabular}{|c|c|c|c|c|c|}
\hline End Conditions & $\begin{array}{c}\text { Xu et al. [195] } \\
\omega_{1}\end{array}$ & $\begin{array}{c}\text { FEM } \\
\omega_{1}\end{array}$ & $\begin{array}{c}\text { DSM } \\
\omega\end{array}$ & $\begin{array}{c}\text { Error1 (\%) } \\
\text { DSM vs. [195] }\end{array}$ & $\begin{array}{c}\text { Error2 (\%) } \\
\text { DSM vs. FEM }\end{array}$ \\
\hline Simply Supported & $4.673 \mathrm{E}+11$ & $4.67289 \mathrm{E}+11$ & $4.6 \mathrm{E}+11$ & $1.6 \%$ & $1.6 \%$ \\
\hline Clamped-Clamped & $1.058 \mathrm{E}+12$ & $1.049 \mathrm{E}+12$ & $1.04 \mathrm{E}+12$ & $0.9 \%$ & $1.7 \%$ \\
\hline Clamped-Free & $1.66501 \mathrm{E}+11$ & $1.67186 \mathrm{E}+11$ & $1.6704 \mathrm{E}+11$ & $0.1 \%$ & $-0.3 \%$ \\
\hline
\end{tabular}

\subsubsection{DWCNTs modeled as local Euler-Bernoulli beam, embedded in an elastic medium}

The natural frequencies for DWCNT modeled as local Euler-Bernoulli beam embedded in an elastic medium are tabulated in Table 4.2. The natural frequencies obtained using the Dynamic Stiffness Matrix (DSM) are compared with those obtained using exact and finite element formulations and the differences are calculated. As can be seen from Table 4.2, the percentage error in this case is always less than $1 \%$. Once again, the results achieved using a single element DSM model are comparable with those obtained using a mesh of around 50 conventional FEM elements. 
Table 4.2 - DSM natural frequencies of TWCNTs, modeled as local Euler-Bernoulli beam, embedded in an elastic medium

\begin{tabular}{|c|c|c|c|c|c|c|}
\hline $\begin{array}{c}\text { End } \\
\text { Conditions }\end{array}$ & $k$ & $\begin{array}{c}\text { Xu et al. [195] } \\
\omega_{1}\left(10^{12} \mathrm{rad} / \mathrm{s}\right)\end{array}$ & $\begin{array}{c}\text { FEM } \omega_{1} \\
\left(10^{12} \mathrm{rad} / \mathrm{s}\right)\end{array}$ & $\begin{array}{c}\text { DSM } \omega \\
\left(10^{12} \mathrm{rad} / \mathrm{s}\right)\end{array}$ & $\begin{array}{c}\text { Error 1 } \\
\text { DSM/[195] }\end{array}$ & $\begin{array}{c}\text { Error 2 } \\
\text { DSM/FEM }\end{array}$ \\
\hline \multirow{4}{*}{$\begin{array}{c}\text { Simply } \\
\text { Supported }\end{array}$} & $k / c=0.1$ & 1.256 & 1.256 & 1.25 & $0.50 \%$ & $0.50 \%$ \\
\cline { 2 - 7 } & $k / c=0.0001$ & 0.468 & 0.468 & 0.468 & $0.16 \%$ & $0.16 \%$ \\
\cline { 2 - 7 } & $k / c=100$ & 6.404 & 6.404 & 6.446 & $-0.66 \%$ & $-0.66 \%$ \\
\hline \multirow{3}{*}{$\begin{array}{c}\text { Clamped } \\
\text { Clamped }\end{array}$} & $k / c=1$ & 3.507 & 3.506 & 3.481 & $0.73 \%$ & $0.73 \%$ \\
\cline { 2 - 7 } & $k / c=0.0001$ & 1.058 & 1.058 & 1.058 & $0.04 \%$ & $0.04 \%$ \\
\cline { 2 - 7 } & $k / c=100$ & 6.398 & 6.431 & 6.397 & $0.52 \%$ & $0.01 \%$ \\
\hline \multirow{3}{*}{$\begin{array}{c}\text { Clamped } \\
\text { Free }\end{array}$} & $k / c=100$ & 6.010 & 6.010 & 6.010 & $-0.00 \%$ & $0.00 \%$ \\
\cline { 2 - 7 } & $k / c=0.1$ & 1.179 & 1.179 & 1.167 & $0.99 \%$ & $0.99 \%$ \\
\cline { 2 - 7 } & $k / c=100$ & 6.483 & 3.483 & 3.480 & $0.10 \%$ & $0.10 \%$ \\
\hline
\end{tabular}

\subsection{Conclusion}

Dynamic Stiffness Matrix (DSM) formulation was developed and used to carry out the free vibration analysis of double-walled carbon nanotubes with and without surrounding elastic medium. The nonlinear eigenvalue problem obtained from the frequency-dependent stiffness matrix was solved using Wittrick-Williams (W-W) algorithm. The natural frequency results for different system's boundary conditions matched those obtained from the conventional FEM and values presented in literature. 


\section{Finite Element Formulation - Triple-Walled Carbon Nanotubes}

\subsection{Introduction}

Studying triple-walled carbon nanotubes offer a great insight into multiwall CNTs with number of walls greater than two. This case and relevant aspects have rarely been studied and most of the research works published in the open literature stops with the double-walled configurations. In this chapter, the conventional finite element formulation for the free vibration analysis of triplewalled carbon nanotubes, using Galerkin weighted residual approach, is developed and the corresponding frequency results are studied. Schematic figure for a TWCNT is given in Figure 5.1 .

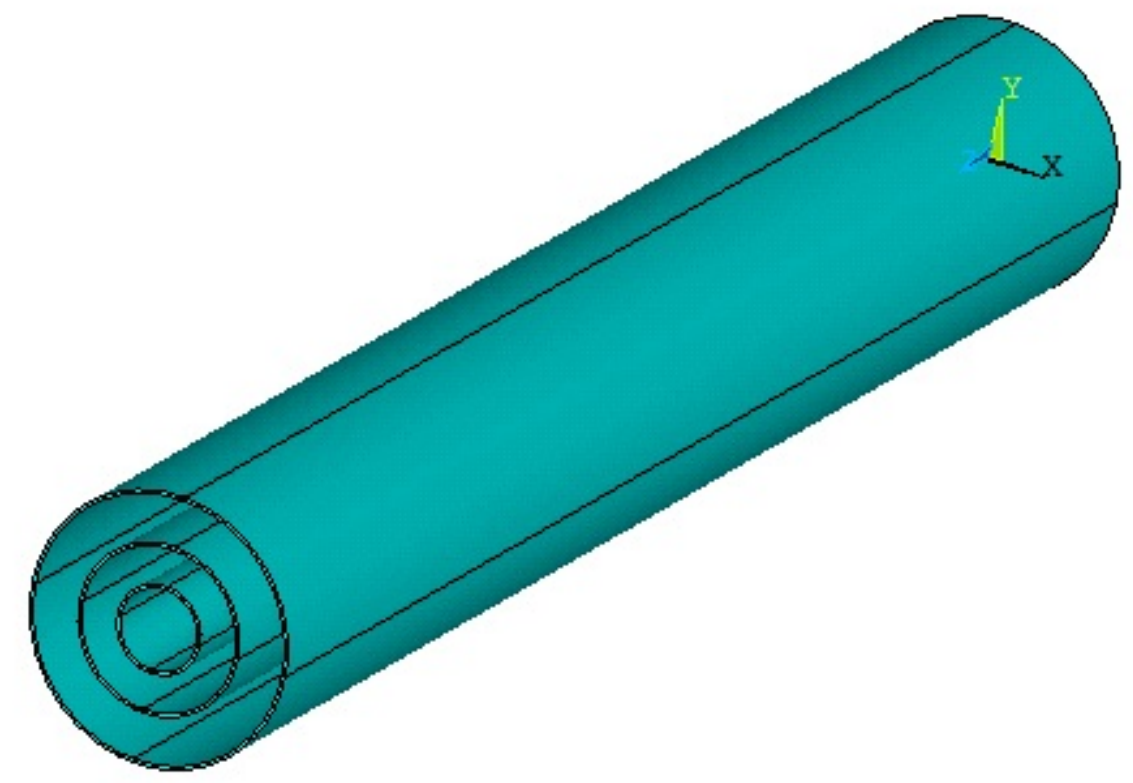

Figure 5.1 - Schematic representation of TWCNT

\subsection{Formulation}

In what follows, triple-walled carbon nanotubes (TWCNTs) are also modeled as local EulerBernoulli beams with van der Waals forces providing the coupling between individual beams. 
Using the equations (3.1) to (3.4) defined earlier, the governing equations for triple-walled carbon nanotubes are defined as [198]:

$$
\begin{gathered}
E_{1} I_{1} w_{1}^{\prime \prime \prime}+\rho_{1} A_{1} \ddot{w}_{1}=c_{1} w_{2}-c_{1} w_{1} \\
E_{2} I_{2} w_{2}^{\prime \prime \prime \prime}+\rho_{2} A_{2} \ddot{w}_{2}=c_{2} w_{3}-c_{2} w_{2}-c_{1} w_{2}+c_{1} w_{1} \\
E_{3} I_{3} w_{3}^{\prime \prime \prime \prime}+\rho_{3} A_{3} \ddot{w}_{3}=-\hat{c}_{2} w_{3}+c_{2} w_{2}
\end{gathered}
$$

where the derivatives with respect to length of the beam and time are denoted by prime ( $)$ and dot (.), respectively. The subscripts 1,2 , and 3 represent the inner, middle and outer carbon nanotubes, respectively. The term $\hat{c}$ in equation (5.3) follows equations (3.7) and (3.8), and can be considered for TWCNTs with and without the surrounding elastic medium. Elimination of time dependency in (5.1), (5.2) and (5.3) is made by assuming simple harmonic motion and using the following transformations, similar to (3.9) and (3.10).

$$
\begin{aligned}
& w_{1}(x, t)=W_{1} e^{i \omega t} \\
& w_{2}(x, t)=W_{2} e^{i \omega t} \\
& w_{3}(x, t)=W_{3} e^{i \omega t}
\end{aligned}
$$

where the transverse displacements of inner, middle and outer tubes, respectively, are represented by $W_{1}, W_{2}$ and $W_{3}$, whereas $\omega$ and $t$ stand for the circular frequency and time. Using these transformations in governing equations (5.1) - (5.3) leads to the following form of the governing equations:

$$
\begin{gathered}
E_{1} I_{1} W_{1}^{\prime \prime \prime \prime}+\left(c_{1}-\rho_{1} A_{1} \omega^{2}\right) W_{1}-c_{1} W_{2}=0 \\
E_{2} I_{2} W^{\prime \prime \prime \prime}+\left(c_{1}+c_{2}-\rho_{2} A_{2} \omega^{2}\right) W_{2}-c_{2} W_{3}-c_{1} W_{2}=0 \\
E_{3} I_{3} W^{\prime \prime \prime \prime}+\left(\hat{c}_{2}-\rho_{3} A_{3} \omega^{2}\right) W_{3}-c_{2} W_{2}=0
\end{gathered}
$$


As can be seen from the above equations, both the inner and outer tubes are coupled with the middle one, as represented by the last terms in (5.7) and (5.9). Further, the middle tube has two coupling terms as it is coupled with both the inner and outer tube, as seen in equation (5.8).

Representing $W_{j}(\mathrm{j}=1,2,3)$ as the amplitude of displacements, and $\delta W_{1,2,3}$ as the weighting functions on the sets of equations, as Galerkin weighted residual method suggests, the integral form of (5.7) - (5.9) is developed as shown below.

$$
\begin{gathered}
\bar{W}_{1}=\int_{0}^{L}\left(E_{1} I_{1} W_{1}^{\prime \prime \prime \prime} \delta W_{1}+\left(c_{1}-\rho_{1} A_{1} \omega^{2}\right) W_{1} \delta W_{1}-c_{1} W_{2} \delta W_{1}\right) d x=0 \\
\bar{W}_{2}=\int_{0}^{L}\left(E_{2} I_{2} W_{2}^{\prime \prime \prime} \delta W_{2}+\left(c_{1}+c_{2}-\rho_{2} A_{2} \omega^{2}\right) W_{2} \delta W_{2}-c_{2} W_{3} \delta W_{2}-c_{1} W_{2} \delta W_{2}\right) d x=0 \\
\bar{W}_{3}=\int_{0}^{L}\left(E_{3} I_{3} W^{\prime \prime \prime \prime} \delta W_{3}+\left(\hat{c}_{2}-\rho_{3} A_{3} \omega^{2}\right) W_{3} \delta W_{3}-c_{2} W_{2} \delta W_{3}\right) d x=0
\end{gathered}
$$

where the subscripts $(1,2$, and 3$)$ represent the inner, middle and outer carbon nanotubes, respectively, both for transverse displacements and weighting functions, as described earlier. The weak integral forms for (5.10) - (5.12) is obtained by integrating them by parts, as shown below.

$$
\begin{gathered}
\bar{W}_{1}=\int_{0}^{L}\left(E_{1} I_{1} W_{1}^{\prime \prime} \delta W_{1}^{\prime \prime}+\left(c_{1}-\rho_{1} A_{1} \omega^{2}\right) W_{1}^{\prime \prime} \delta W_{1}^{\prime \prime}-c_{1} W_{2} \delta W_{1}\right) d x+\left[\left(E_{1} I W_{1}^{\prime \prime \prime}\right) \delta W_{1}\right]_{0}^{L}-\left[\left(E_{1} I_{1} W_{1}^{\prime \prime}\right) \delta W_{1}^{\prime}\right]_{0}^{L}=0 \\
\bar{W}_{2}=\int_{0}^{L}\left(E_{2} I_{2} W_{2}^{\prime \prime} \delta W_{2}^{\prime \prime}+\left(c_{1}+c_{2}-\rho_{2} A_{2} \omega^{2}\right) W_{2}^{\prime \prime} \delta W_{2}^{\prime \prime}-c_{2} W_{3} \delta W_{2}-c_{1} W_{2} \delta W_{2}\right) d x+\left\lfloor\left(E_{2} I_{2} W_{2}^{\prime \prime \prime}\right) \delta W_{2}\right]_{0}^{L} \\
-\left[\left(E_{2} I_{2} W_{2}^{\prime \prime}\right) \delta W_{2}^{\prime}\right]_{0}^{L}=0
\end{gathered}
$$

$\bar{W}_{3}=\int_{0}^{L}\left(E_{3} I_{3} W_{3}^{\prime \prime} \delta W_{3}^{\prime \prime}+\left(\widehat{c}_{2}-\rho_{3} A_{3} \omega^{2}\right) W_{3}^{\prime \prime} \delta W_{3}^{\prime \prime}-c_{2} W_{2} \delta W_{3}\right) d x+\left[\left(E_{3} I_{3} W_{3}^{\prime \prime \prime}\right) \delta W_{3}\right]_{0}^{L}-\left[\left(E_{3} I_{3} W_{3}^{\prime \prime}\right) \delta W_{1}^{\prime}\right]_{0}^{L}=0$

The strikethrough terms in the above equations represent the boundary terms going to zero with the application of the system's boundary conditions. The system is now discretized using a 
number $(k)$ of six-node line elements, with 2 degrees-of-freedom per node, as opposed to 4-node elements for double-walled CNT. The discretized, i.e., element integral equations are written as:

$$
\begin{gathered}
W_{w_{1}}^{k}=\int_{0}^{l^{k}} \delta W_{1}^{\prime \prime}\left(E_{1} I_{1} W_{1}^{\prime \prime}\right) d x+\int_{0}^{l^{k}} \delta W_{1}^{\prime \prime}\left(c_{1}-\rho_{1} A_{1} \omega^{2}\right) W_{1}-\int_{0}^{l^{k}} \delta W_{1} c W_{2} \\
W_{w_{2}}^{k}=\int_{0}^{l^{k}} \delta W_{2}^{\prime \prime}\left(E_{2} I_{2} W_{2}^{\prime \prime}\right) d x+\int_{0}^{l^{k}} \delta W_{2}^{\prime \prime}\left(c_{1}+c_{2}-\rho_{2} A_{2} \omega^{2}\right) W_{2}-\int_{0}^{l^{k}} \delta W_{2} c_{2} W_{3}-\int_{0}^{l^{k}} \delta W_{2} c_{1} W_{2} \\
W_{w_{3}}^{k}=\int_{0}^{l^{k}} \delta W_{3}^{\prime \prime}\left(E_{3} I_{3} W_{3}^{\prime \prime}\right) d x+\int_{0}^{l^{k}} \delta W_{1}^{\prime \prime}\left(\widehat{c}_{2}-\rho_{3} A_{3} \omega^{2}\right) W_{3}-\int_{0}^{l^{k}} \delta W_{3} c_{2} W_{2}
\end{gathered}
$$

For each carbon nanotube represented in equations (5.16) - (5.18), Hermite type polynomial approximations are used to express approximate lateral displacements in terms of nodal values. The stiffness, mass, semi-mass and coupling matrices are similar to ones represented in equation (3.24). For a 2 DOF per node element, one follows the expressions (3.19) - (3.23) to obtain the uncoupled matrices for triple-walled carbon nanotubes given by equations (5.19) - (5.21).

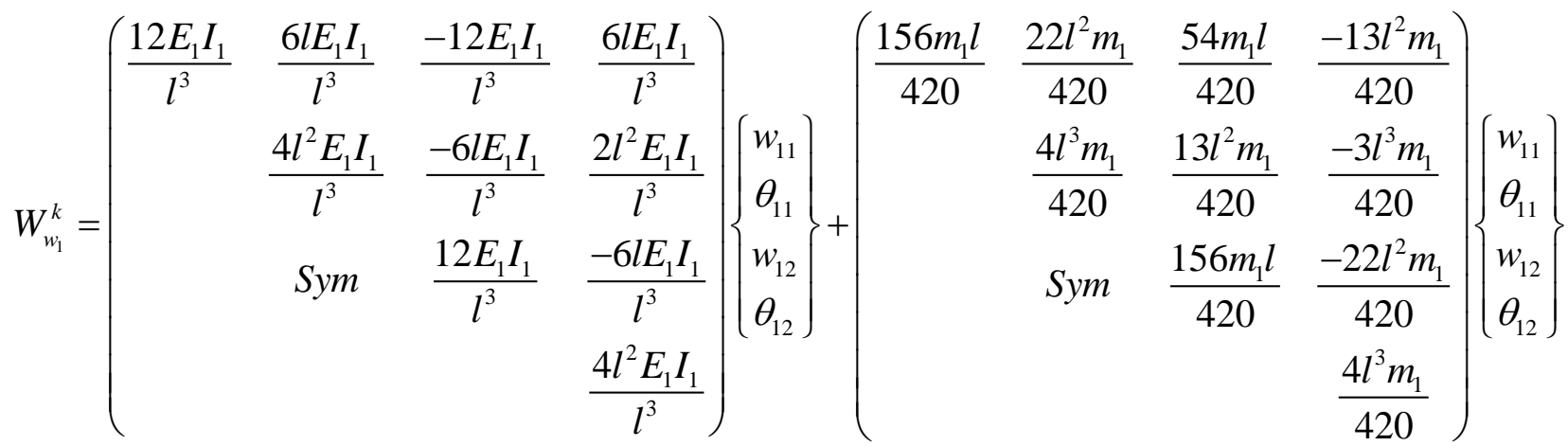

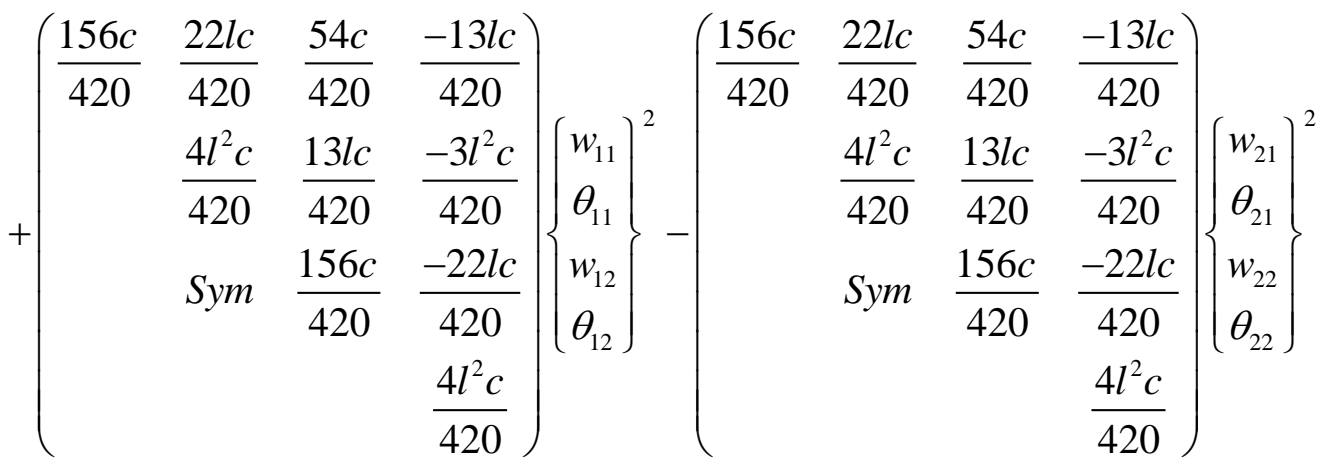




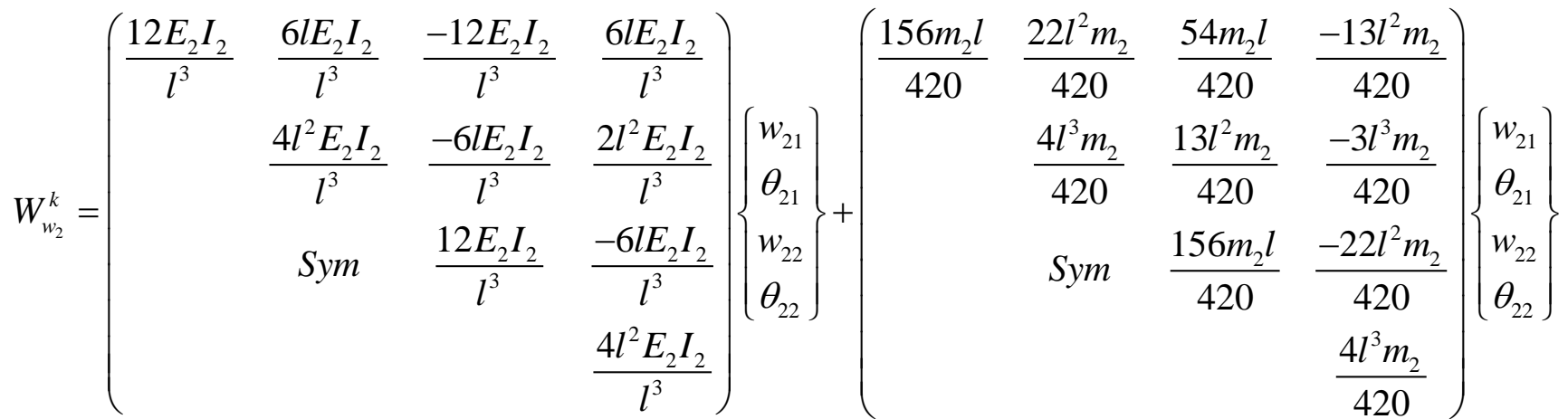

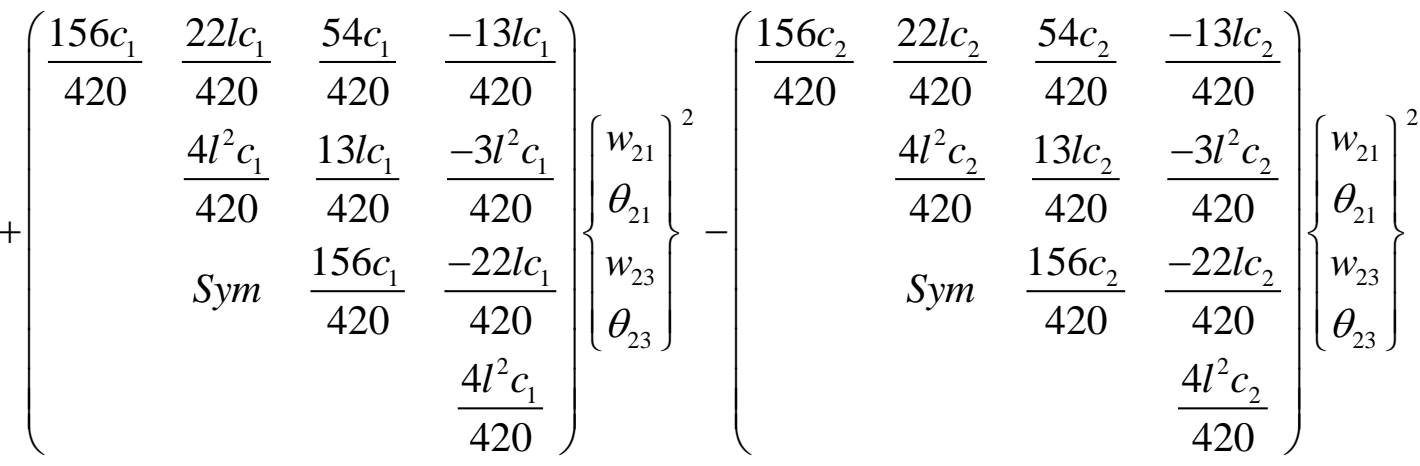

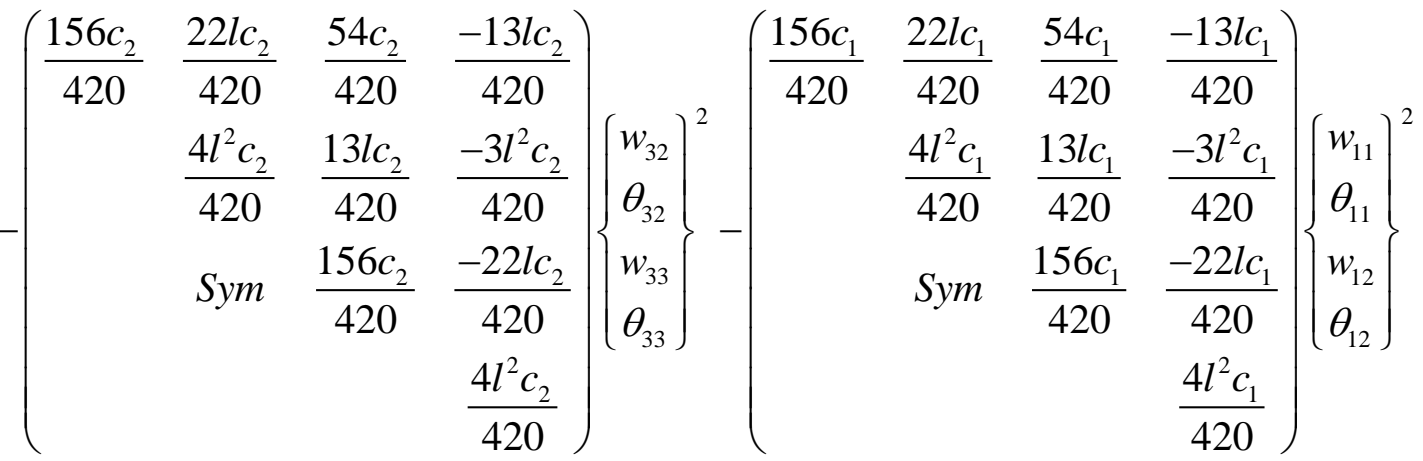




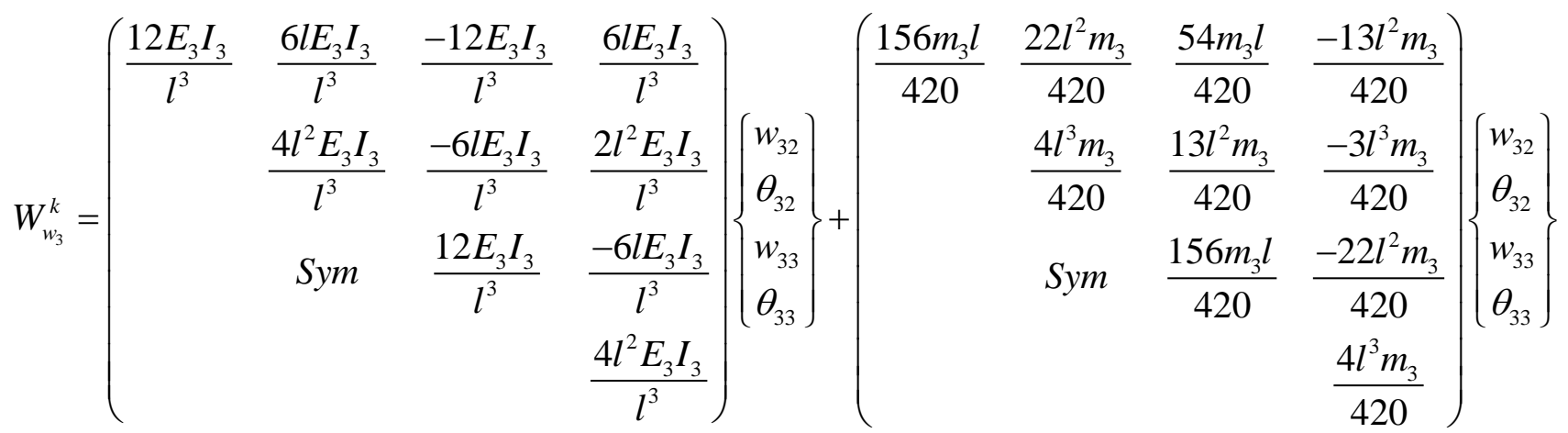

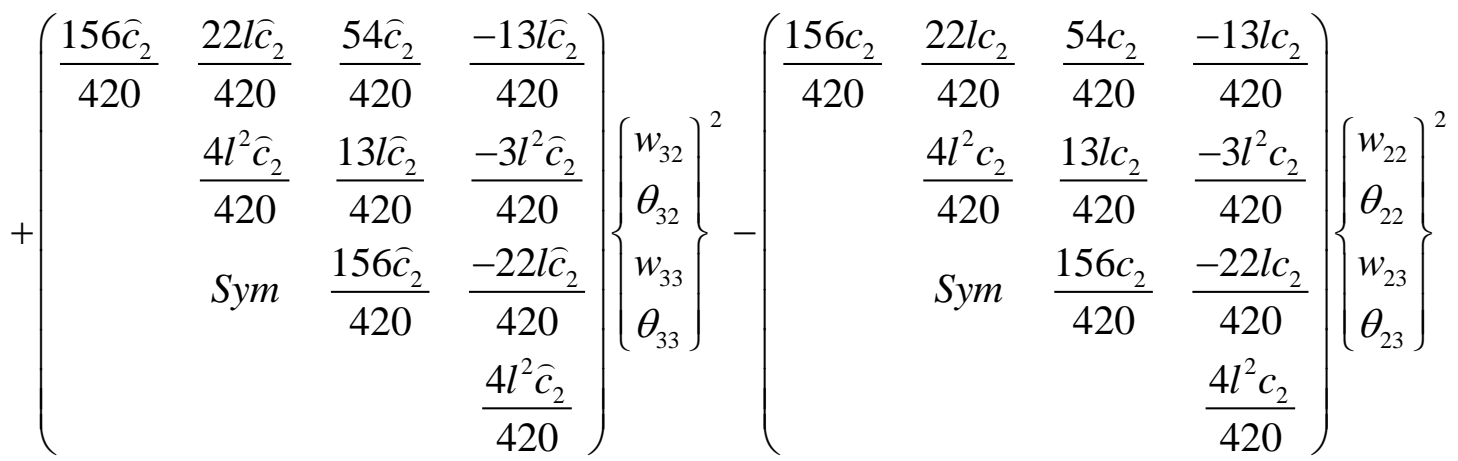

The elemental stiffness and mass matrices of the TWCNT are represented as $\left[k_{t w}\right]$ and $\left[m_{t w]}\right.$, respectively, and have been presented in Appendix II. The linear eigenvalue problem, represented by (5.22) below, is obtained by assembling the element matrices and enforcing the system’s boundary conditions, and is solved using a MATLAB ${ }^{\circledR}$ code.

$$
\operatorname{det}\left(K_{t w}-\omega^{2} M_{t w}\right)=0
$$

where $K_{t w}$ and $M_{t w}$ are the system's global (overall) stiffness and mass matrices, respectively.

\subsection{Results and Discussion}

In this section, the application of the FEM formulation presented above is demonstrated through the free vibration analysis of illustrative TWCNT examples, where the following geometric and material properties and dimensions, reported by Elishakoff et al. [198], are used: 


$$
\begin{array}{cc}
L=14 e-9, & \rho_{1}=\rho_{2}=\rho_{3}=2.3 \frac{\mathrm{g}}{\mathrm{cm}^{3}} \\
E_{1}=E_{2}=E_{3}=1 \mathrm{TPa}, & c_{1}=69.43 \mathrm{GPa} ; c_{2}=138.86 \mathrm{GPa} \\
\left.r_{1, i} \text { (inner radius, } \text { inner tube }\right)=0.18 \mathrm{~nm}, & \left.r_{1, o} \text { (outer radius, } \text { inner tube }\right)=0.52 \mathrm{~nm}, \\
\left.r_{2, i} \text { (inner radius, middle tube }\right)=0.53 \mathrm{~nm}, & \left.r_{2, o} \text { (outer radius, middle tube }\right)=0.87 \mathrm{~nm} \\
\left.r_{3, i} \text { (inner radius, outer tube }\right)=0.88 \mathrm{~nm}, & \left.r_{3, o} \text { (outer radius, outer tube }\right)=1.22 \mathrm{~nm}
\end{array}
$$

\subsubsection{Triple-walled Carbon Nanotubes, modeled as local Euler-Bernoulli beam}

The natural frequencies of TWCNTs for three different boundary conditions are given in Table 5.1. This study uses the Finite Element Method (FEM). The convergence is studied by increasing the number of elements and plotting the error percentage either going to zero or becoming stable. As far as the author's knowledge goes, there are no exact methods present for validation of the natural frequencies of TWCNTs and so this study compared the first natural frequency of the DWCNTs, found earlier in Chapter 3. The ratio of natural frequency of TWCNTs to that of DWCNTs was found to be constant for all boundary conditions, hence validating the formulation of TWCNTs.

Even though the convergence is achieved for a courser mesh, this study considers the natural frequencies for 30-element model. The figures following the Table 5.1 graph the convergence plot between the number of elements and error percentage for the three beams of the TWCNTs, with all the boundary conditions.

Table 5.2 compares the nondimentional natural frequencies of individual tubes of TWCNT, with those of TWCNT, obtained using the single beam model, as explained by Equation (3.29), which involves summing up of the geometric and material properties of individual tubes and representing them as $I, A$ and $E$ respectively, as considered by Yoon et al. [41]. The nondimentional natural frequencies are obtained using (3.30) given in Chapter 3. 
Table 5.1 - FEM natural Frequencies of TWCNTs, modeled as local Euler-Bernoulli beam

\begin{tabular}{|c|c|c|c|}
\hline Boundary Conditions & $\begin{array}{c}\omega_{1} \\
\left(10^{12} \mathrm{rad} / \mathrm{s}\right)\end{array}$ & $\begin{array}{c}\omega_{2} \\
\left(10^{12} \mathrm{rad} / \mathrm{s}\right)\end{array}$ & $\begin{array}{c}\omega_{3} \\
\left(10^{12} \mathrm{rad} / \mathrm{s}\right)\end{array}$ \\
\hline Simply Supported & 0.647 & 2.531 & 5.038 \\
\hline Clamped-Clamped & 1.459 & 3.793 & 6.049 \\
\hline Clamped-Free & 0.231 & 1.438 & 3.795 \\
\hline
\end{tabular}

Table 5.2 - Comparison of the non-dimensionalized FEM natural frequencies of a SWCNT with those of TWCNT, both modeled as local Euler-Bernoulli beam

\begin{tabular}{|c|c|c|c|c|}
\hline \multirow{2}{*}{$\begin{array}{c}\text { Boundary } \\
\text { Conditions }\end{array}$} & $\begin{array}{c}\text { TWCNT } \\
\text { (Inner Tube) }\end{array}$ & $\begin{array}{c}\text { TWCNT } \\
\text { (Middle Tube) }\end{array}$ & $\begin{array}{c}\text { TWCNT } \\
\text { (Outer Tube) }\end{array}$ & $\begin{array}{c}\text { Single Beam } \\
\text { Model }\end{array}$ \\
\cline { 2 - 5 } & $\omega_{11}$ & $\omega_{12}$ & $\omega_{13}$ & $\omega$ \\
\hline Simply-Supported & 22.1369 & 109.8748 & 120.5045 & 9.8696 \\
\hline Clamped-Clamped & 49.8699 & 111.6264 & 121.3185 & 22.3733 \\
\hline Clamped-Free & 7.8963 & 109.5073 & 120.3333 & 3.5160 \\
\hline
\end{tabular}

In Table 5.1, $\omega_{1}, \omega_{2}$, and $\omega_{3}$ represent the first three natural frequencies of the system and $\omega_{11}$, $\omega_{12}$ and $\omega_{13}$ in Table 5.2 represent the nondimentionalized natural frequency of inner, middle and outer tube, respectively. The convergence plots are graphed in Figures (5.2) - (5.4), which plot the number of elements on $\mathrm{x}$-axis and the error percentage on $\mathrm{y}$-axis. 


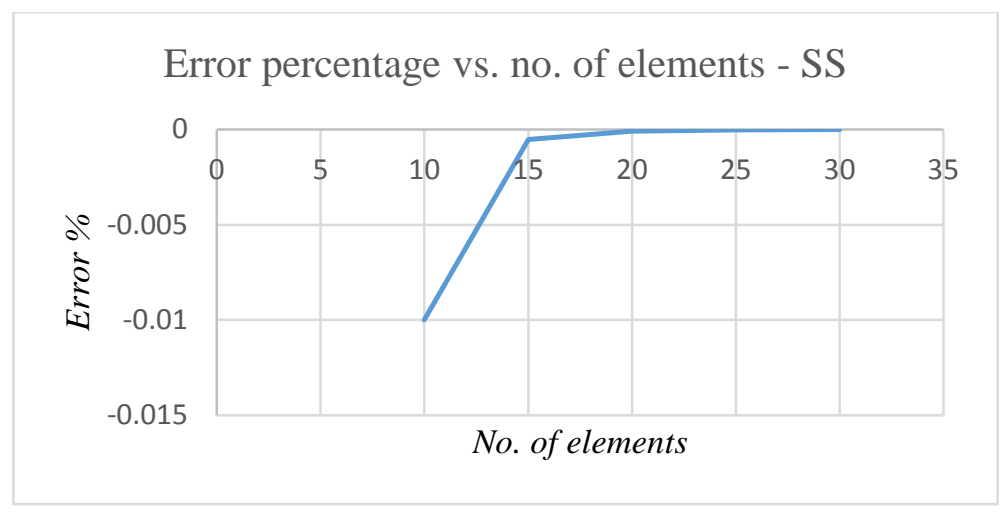

Figure 5.2 - Percentage error vs. the number of elements - SS - Up to 30 FEM elements

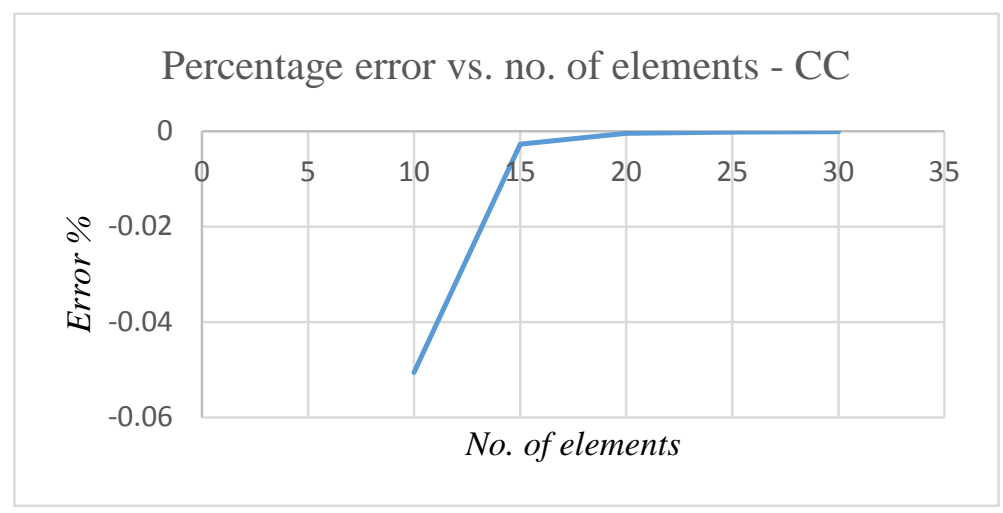

Figure 5.3 - Percentage error vs. the number of elements - CC - Up to 30 FEM elements

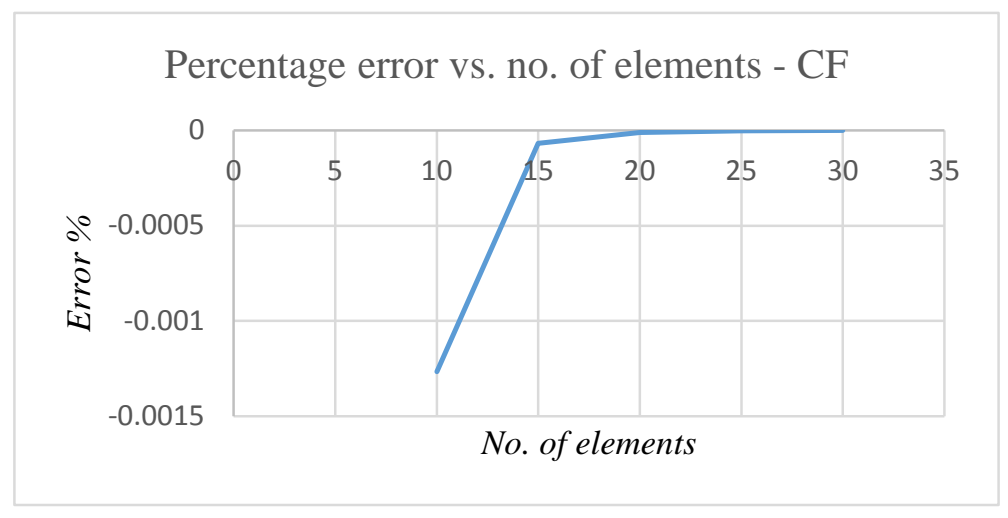

Figure 5.4 - Percentage error vs. the number of elements - CF - Up to 30 FEM elements 
The mode shapes for the free vibration of a TWCNT are given in Figures (5.5) - (5.7).

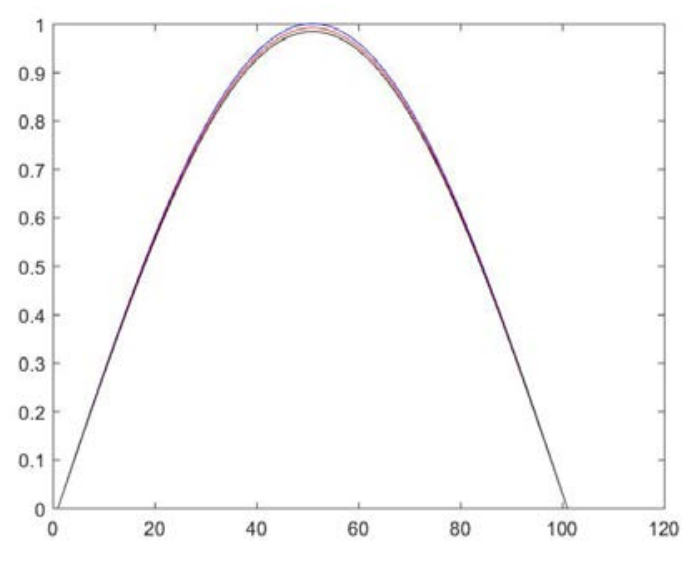

Mode 1

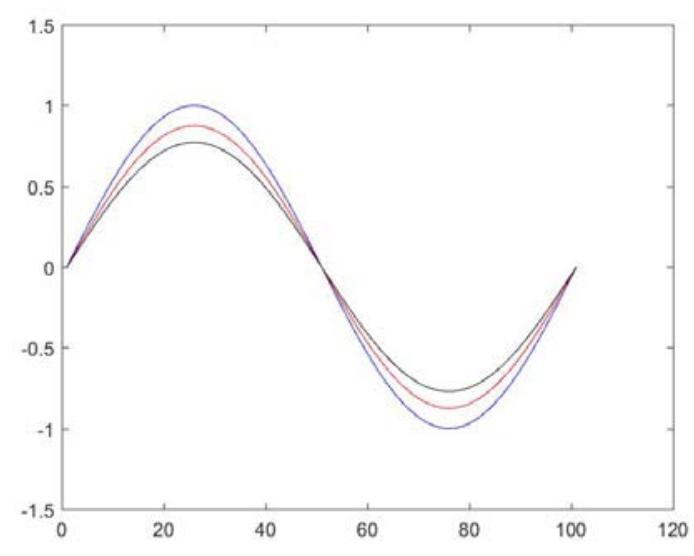

Mode 2

Figure 5.5 - First two mode shapes of TWCNT modeled as local Euler-Bernoulli beam - simply supported boundary condition - FEM

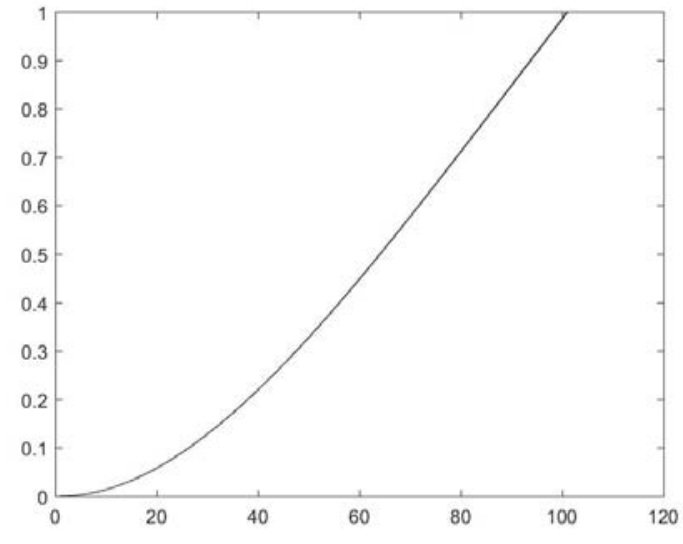

Mode 1

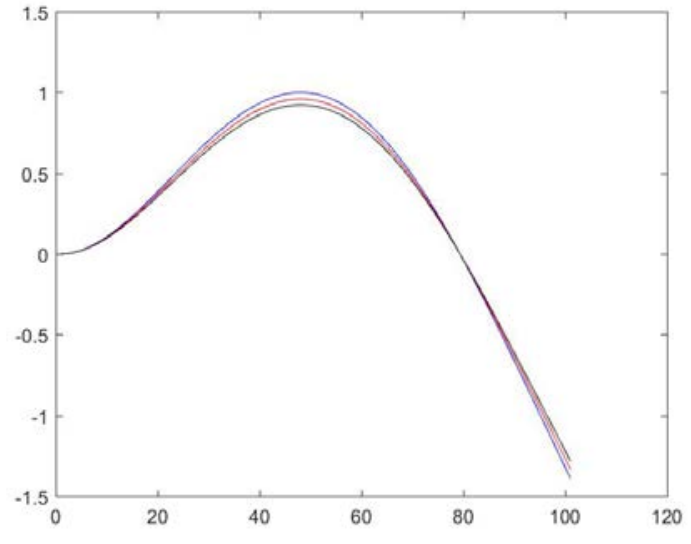

Mode 2

Figure 5.6 - First two mode shapes of TWCNT modeled as local Euler-Bernoulli beam clamped-free boundary condition - FEM 


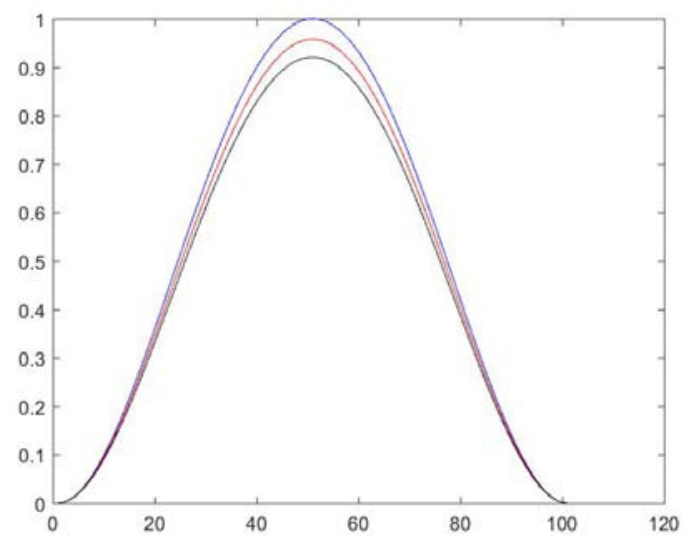

Mode 1

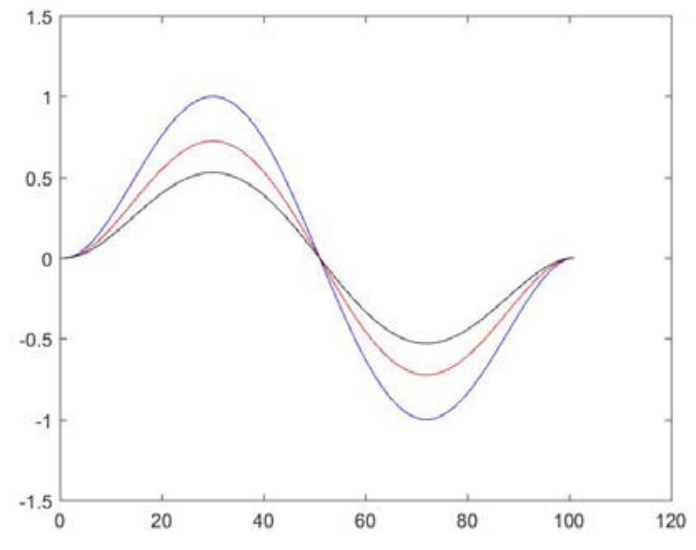

Mode 2

Figure 5.7 - First two mode shapes of TWCNT modeled as local Euler-Bernoulli beam clamped-clamped boundary condition - FEM

\subsubsection{Triple-walled Carbon Nanotubes modeled as local Euler-Bernoulli beam, embedded in an elastic medium}

The natural frequencies of a TWCNT embedded in an elastic medium are presented in Table 5.3. In addition to $k / c=1$ ratio, three other values are taken to represent the effect of various stiffness values of surrounding medium on the vibration response of the TWCNT; i.e., the $k / c$ is either $\quad \gg 1, \ll 1$ or $<1$. The natural frequencies are evaluated using a 30-element FEM model and convergence plots are presented in Figures (5.8) - (5.10). The convergence plots are graphed by taking the error percentages on the $\mathrm{y}$-axis and number of elements on the $\mathrm{x}$-axis.

The figures following Table 5.3 are plotted to show the convergence of the natural frequencies presented in it. The graphs plot the variation of error percentage when the number of elements are increased from 5 to 30 with a step of 5 elements each. It can be seen that the values converge for 30 elements for all the boundary conditions. 
Table 5.3 - FEM natural frequencies of TWCNTs modeled as local Euler-Bernoulli beam embedded in an elastic medium:

\begin{tabular}{|c|c|c|c|c|}
\hline $\begin{array}{c}\text { Boundary } \\
\text { Conditions }\end{array}$ & $k / c$ ratio & $\begin{array}{c}\omega_{1} \\
\left(10^{12} \mathrm{rad} / \mathrm{s}\right)\end{array}$ & $\begin{array}{c}\omega_{2} \\
\left(10^{12} \mathrm{rad} / \mathrm{s}\right)\end{array}$ & $\begin{array}{c}\omega_{3} \\
\left(10^{12} \mathrm{rad} / \mathrm{s}\right)\end{array}$ \\
\hline \multirow{3}{*}{$\begin{array}{c}\text { Simply } \\
\text { Supported }\end{array}$} & $k / c=100$ & 4.495 & 4.737 & 5.606 \\
\cline { 2 - 5 } & $k / c=1$ & 3.139 & 3.737 & 5.261 \\
\cline { 2 - 5 } & $k / c=0.1$ & 1.308 & 2.735 & 5.071 \\
\cline { 2 - 5 } Clamped- & $k / c=0.0001$ & 0.649 & 2.531 & 5.038 \\
\cline { 2 - 5 } Clamped & $k / c=100$ & 4.564 & 5.074 & 6.323 \\
\cline { 2 - 5 } & $k / c=1$ & 3.321 & 4.413 & 6.179 \\
\cline { 2 - 5 } & $k / c=0.1$ & 1.834 & 3.891 & 6.130 \\
\hline \multirow{3}{*}{ Clamped-Free } & $k / c=1001$ & 1.460 & 3.794 & 6.049 \\
\cline { 2 - 5 } & $k / c=1$ & 4.480 & 4.561 & 5.075 \\
\cline { 2 - 5 } & $k / c=0.1$ & 1.165 & 3.315 & 4.414 \\
\cline { 2 - 5 } & $k / c=0.0001$ & 0.234 & 1.817 & 3.892 \\
\hline
\end{tabular}

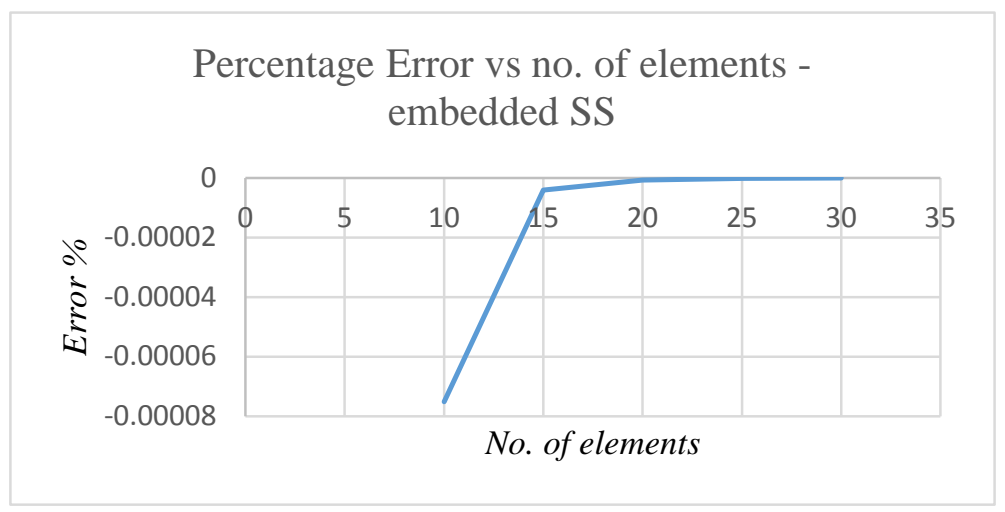

Figure 5.8 - Percentage error vs. the number of elements - embedded SS - 30 FEM elements 


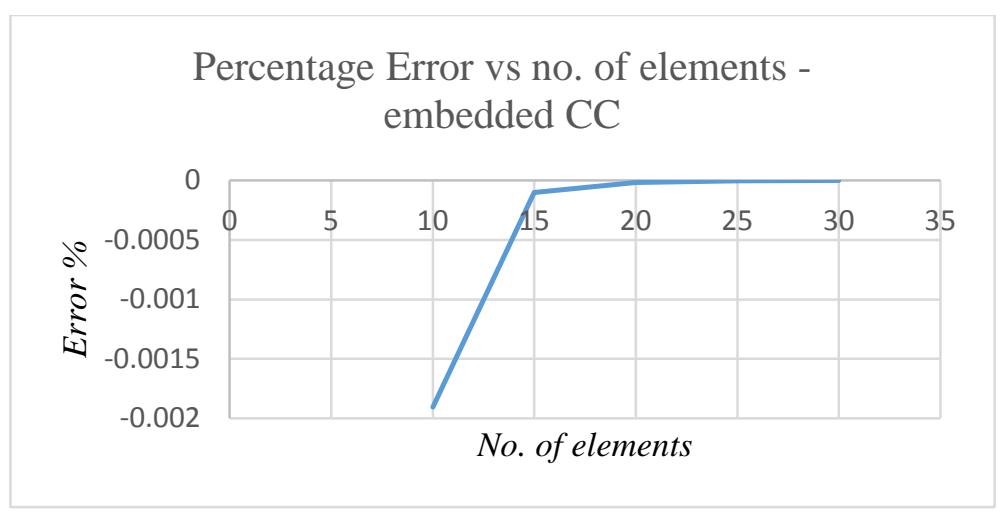

Figure 5.9 - Percentage error vs. the number of elements - embedded CC - 30 FEM elements

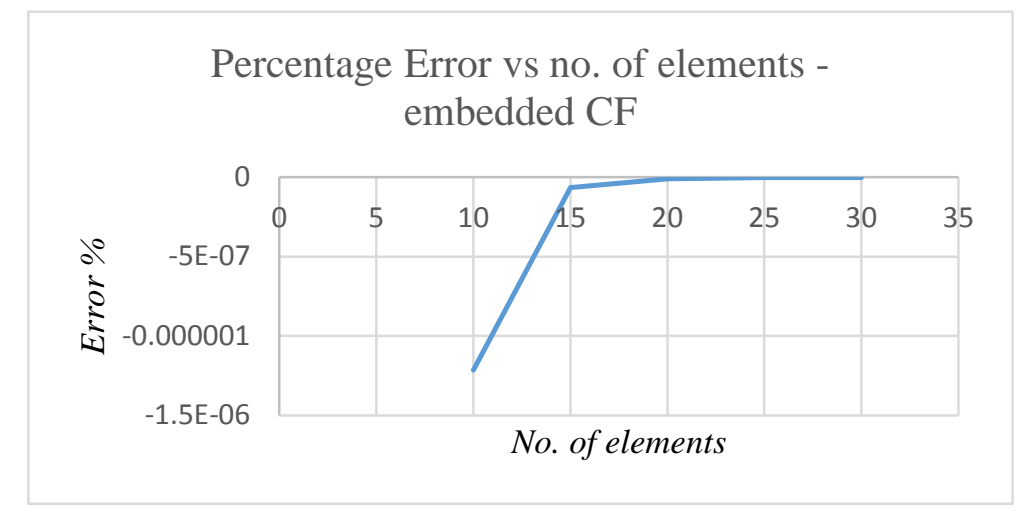

Figure 5.10 - Percentage error vs. the number of elements - embedded CF - 30 FEM elements

The mode shapes for all the boundary conditions for $k / c=0.1$ are presented in Figures (5.11) (5.13). Mode shapes for other cases are presented in Appendix A7. 

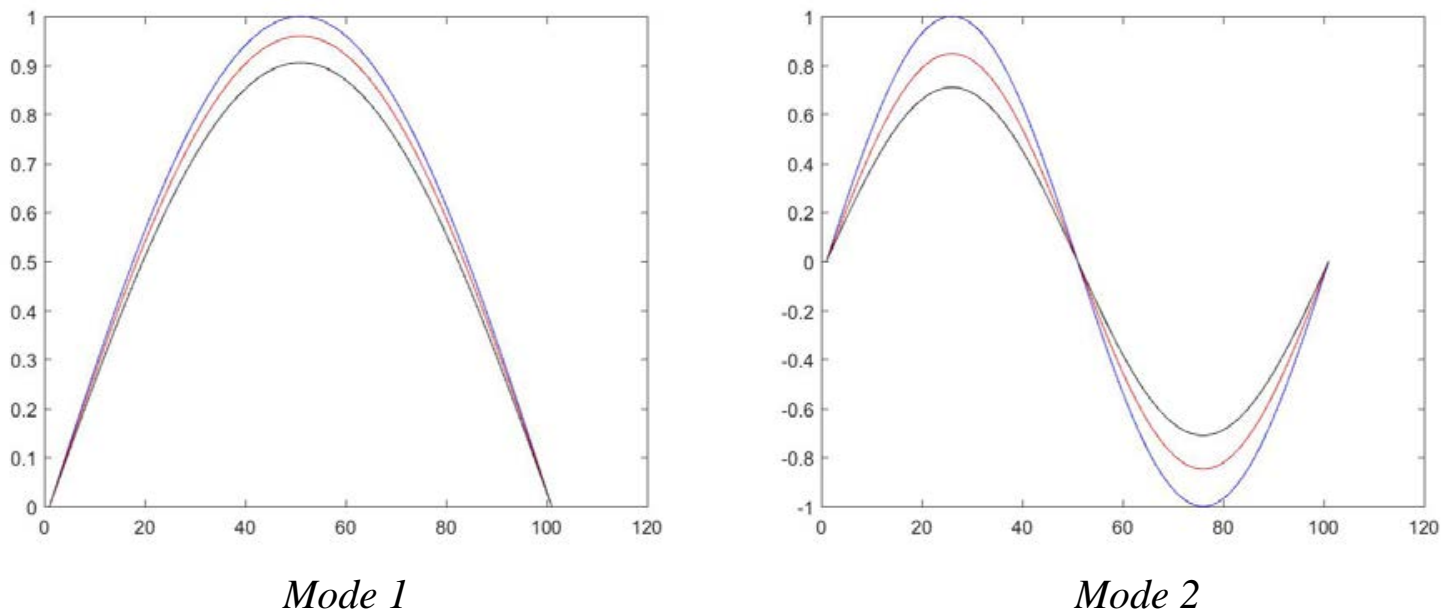

Figure 5.11 - First two mode shapes of TWCNT, modeled as local Euler-Bernoulli beam, embedded in elastic medium $(k / c=0.1)$ - simply supported boundary condition - FEM

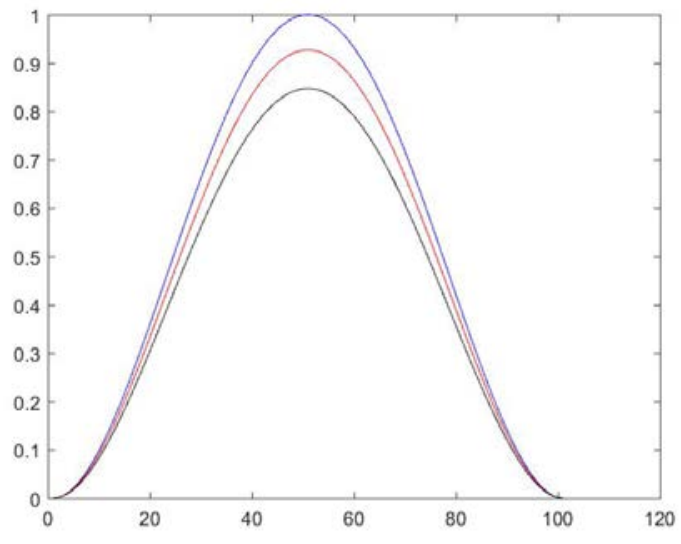

Mode 1

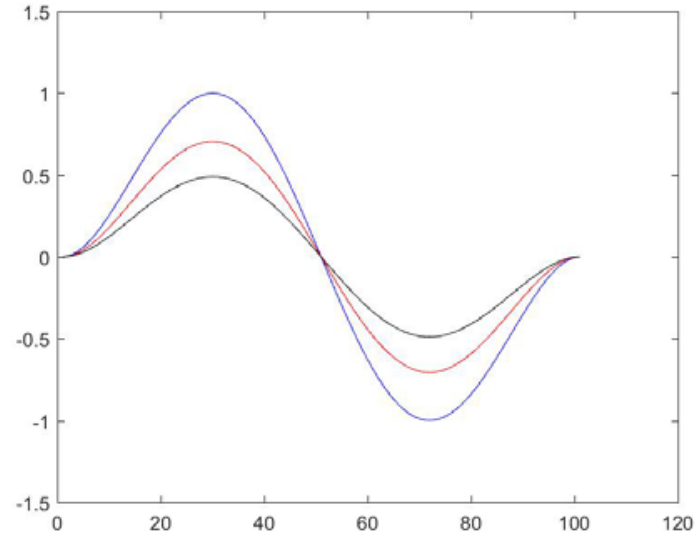

Mode 2

Figure 5.12 - First two mode shapes of TWCNT, modeled as local Euler-Bernoulli beam, embedded in elastic medium $(\mathrm{k} / \mathrm{c}=0.1)$ - clamped-clamped boundary condition - FEM 


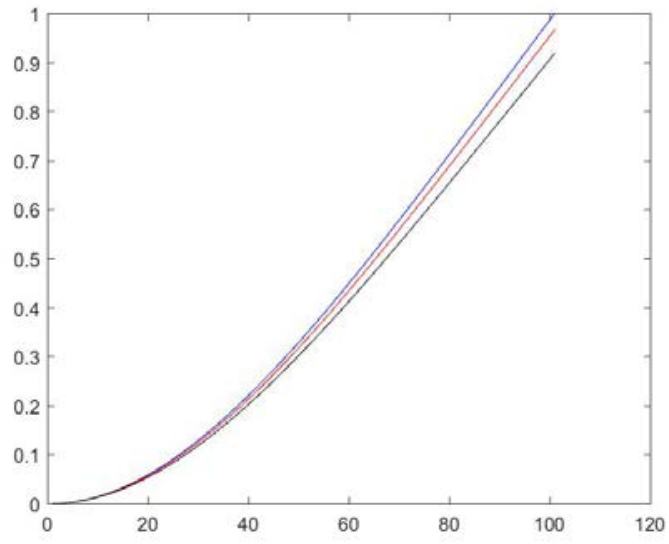

Mode 1

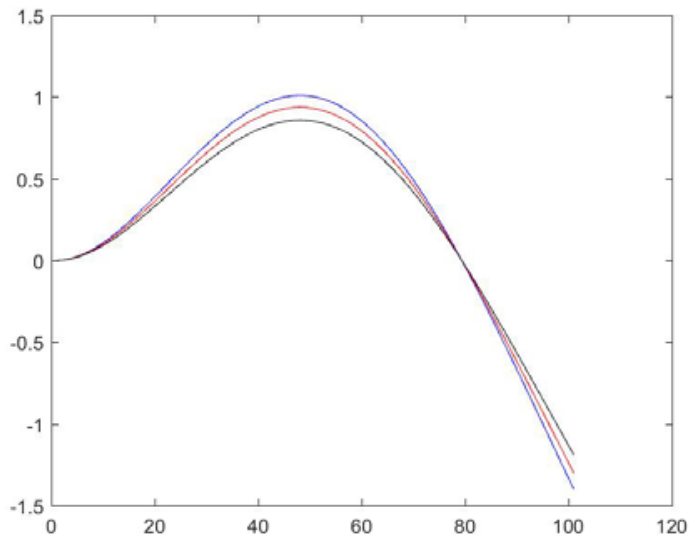

Mode 2

Figure 5.13 - First two mode shapes of TWCNT, modeled as local Euler-Bernoulli beam, embedded in elastic medium $(k / c=0.1)$ - clamped-free boundary condition - FEM

\subsection{Conclusion}

Finite element formulation was applied to triple-walled carbon nanotubes (TWCNTs), both with and without various $(k / c)$ surrounding elastic medium matrix. The resulting linear eigenvalue problem is solved using a program code developed in MATLAB ${ }^{\circledR}$. In addition, an investigation into the mode shapes for all the boundary conditions showed that they do not change with the stiffness of surrounding elastic medium matrix. 


\section{Nonlocal Finite Element Formulation}

\subsection{Introduction}

The nonlocal elasticity presented by Eringen [23] accounts for small scale effects by taking the stress at one point as a reference to be a function of strain at all points in the field. The small scale effect parameter for carbon nanotubes is presented in the terms of a constant value, which is a product of internal characteristic length represented as $a$, and a material specific constant value, $e_{0}$. Zhang et al. [56] used the ratio of theoretical local result and experimental molecular mechanics simulation to arrive at a certain value of $e_{0} \approx 0.82$. The characteristic length, $a$, is taken to be $0.142 \mathrm{~nm}$ i.e., the value of C-C bond length. The nonlocal parameter or small scale effect parameter is given as:

$$
\mu=e_{0} a
$$

Hooke's law is modified using the theory of nonlocal elasticity, written as [61]:

$$
\sigma-(\mu)^{2} \frac{\partial^{2} \sigma}{\partial x^{2}}=E \varepsilon
$$

where $\sigma$ is axial stress and $\varepsilon$ is the axial strain, written for small deflections as [56]

$$
\varepsilon=-y \frac{\partial^{2} y}{\partial x^{2}}
$$

Using (6.2) and (6.3), along with shear and bending moment expressions for a beam, the governing equation for a nonlocal Euler-Bernoulli beam is derived, as briefly discussed in the following section.

\subsection{Formulation}

The formulation of finite element model for nonlocal Euler-Bernoulli beam, along with that of a classical plate, have been presented by Phadikar and Pradhan [199]. This section will exploit the 
same method to derive the mass and stiffness matrices for a single carbon nanotube modelled as a nonlocal Euler-Bernoulli beam. The FEM formulations will then be extended to nonlocal double- and tripled-walled CNTs, which, to the best of author's knowledge, have not been reported in the open literature. It can be seen from previous local CNTs finite element formulations that the presence of coupling terms would not affect the mass matrix, but rather the stiffness. However, as it will be seen later in this chapter, the small scale effect factor only influences the mass matrix. The governing equation of a nonlocal Euler-Bernoulli beam, as reported in [200], is written as:

$$
E I \frac{\partial^{4} w}{\partial x^{4}}-m \omega^{2} w+\mu^{2} m \omega^{2} \frac{\partial^{2} w}{\partial x^{2}}=0
$$

where $E$ is the Young's modulus, $I$ is the cross-sectional $2^{\text {nd }}$ area moment of inertia, $m$ is the mass per unit length $(\rho A), \omega$ is the natural frequency, and $\mu=e_{0} a$, as defined earlier. The moment and shear forces of a nonlocal Euler-Bernoulli beam are given by:

$$
\begin{gathered}
M=-\mu^{2} m \omega^{2}-E I \frac{\partial^{2} w}{\partial x^{2}} \\
V=-\mu^{2} \omega^{2} \frac{\partial^{2} w}{\partial x^{2}}-E I \frac{\partial^{3} w}{\partial x^{3}}
\end{gathered}
$$

After deriving the functional using calculus of variation, and applying integration by parts to the third term, following equation is obtained [199]:

$$
\int_{0}^{l}\left[E I \frac{d^{2} W}{d x^{2}} \frac{d^{2} \chi}{d x^{2}}+\mu^{2} m \omega^{2} \frac{d^{2} W}{d x^{2}} \chi-m \omega^{2} W \chi\right] d x-[\chi V]_{0}^{l}+\left[\frac{d \chi}{d x} M\right]_{0}^{l}=0
$$

where $\chi$ is the weighting function and $W$ is the transverse displacement, The nodal degrees of freedom are taken as $u_{i}$ and the corresponding shape functions, Hermite cubic interpolation functions, are represented by $N_{i}$ thus 


$$
W(x)=\sum_{1}^{4} N_{i} u_{i}
$$

where $N_{i}(i=1,2,3,4)$ are given by

$$
\begin{gathered}
N_{1}=\frac{1}{L^{3}}\left(2 x^{3}-3 x^{2} L+L^{3}\right) \\
N_{2}=\frac{1}{L^{3}}\left(x^{3} L-2 x^{2} L+x L\right) \\
N_{3}=\frac{1}{L^{3}}\left(-2 x^{3}+3 x^{2} L\right) \\
N_{4}=\frac{1}{L^{3}}\left(x^{3} L-x^{2} L^{2}\right)
\end{gathered}
$$

and $u_{i}$ is given as

$$
\left(u_{2 n-1}, u_{2 n}\right)=\left(W,-\frac{d W}{d x}\right)
$$

Following the same FEM formulation as presented in earlier chapters of this thesis document, and taking into account the small scale effect factor he element mass and stiffness matrices, represented as $k$ and $m$, and are derived and are presented below:

$$
k=\frac{E I}{l^{3}}\left[\begin{array}{cccc}
12 & 6 l & -12 & 6 l \\
6 l & 4 l^{2} & -6 l & 2 l^{2} \\
-12 & -6 l & 12 & -6 l \\
6 l & 2 l^{2} & -6 l & 4 l^{2}
\end{array}\right]
$$


It is to be noticed that equation (6.11) is similar to stiffness terms in equations (3.24) and (3.25), with the only difference of subscripts. However, when compared to previous nonlocal mass matrices, expression (6.12) has been modified to incorporate the small scale effects, appearing as a second matrix. Therefore, replacement of mass terms in both equations (3.24) and (3.25) with (6.12) would permit the local Euler-Bernoulli beam double-walled carbon nanotubes formulation to take the small scale effect into consideration, hence transforming into nonlocal Euler-Bernoulli beam double-walled carbon nanotubes. The element matrix equations, with the additional matrix terms representing the small scale factor, are then written as:

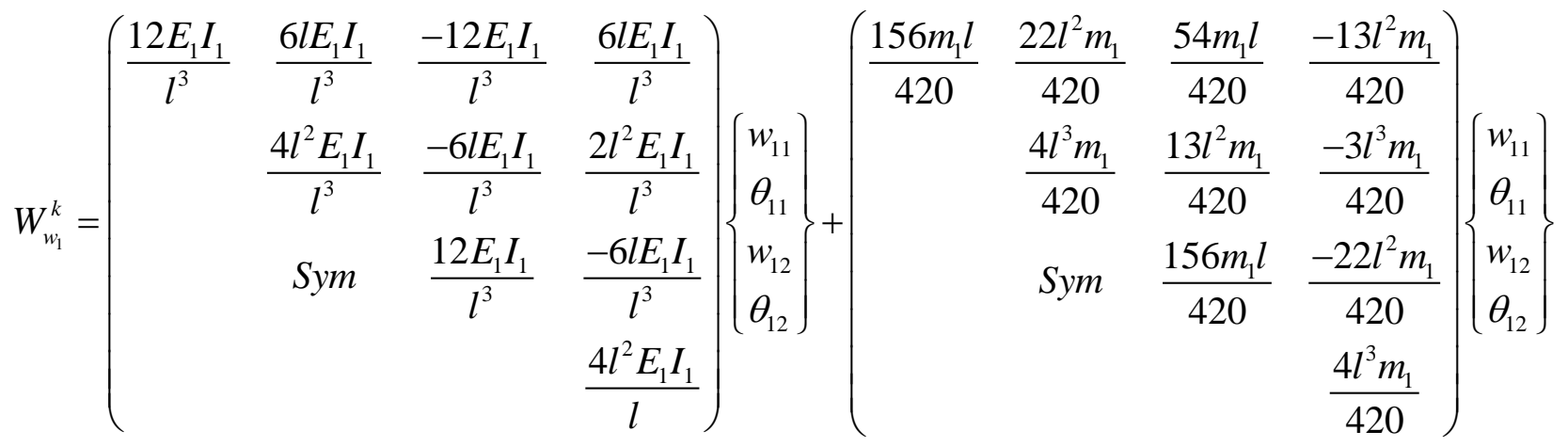

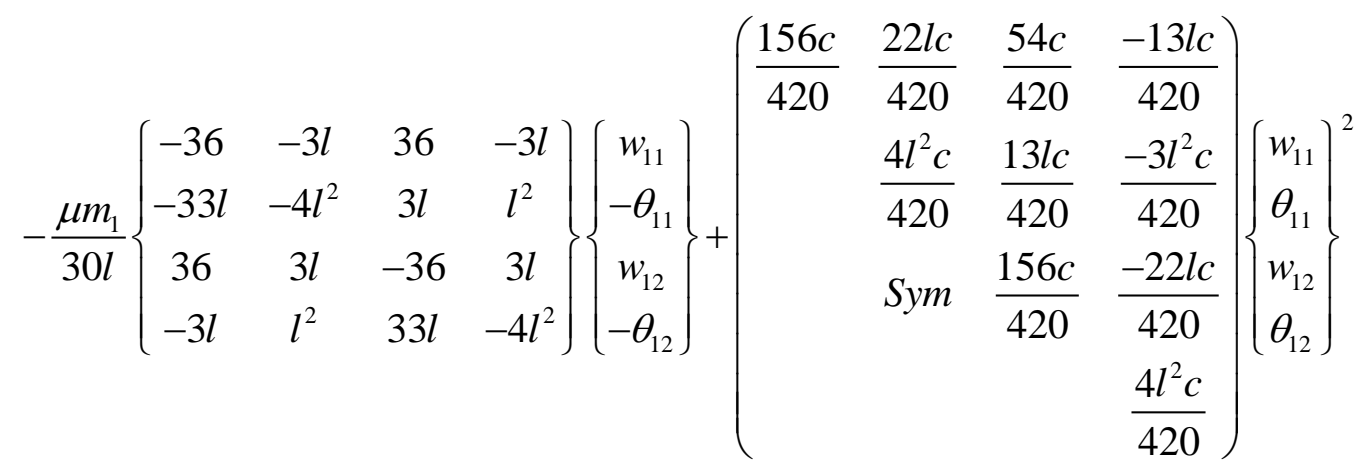

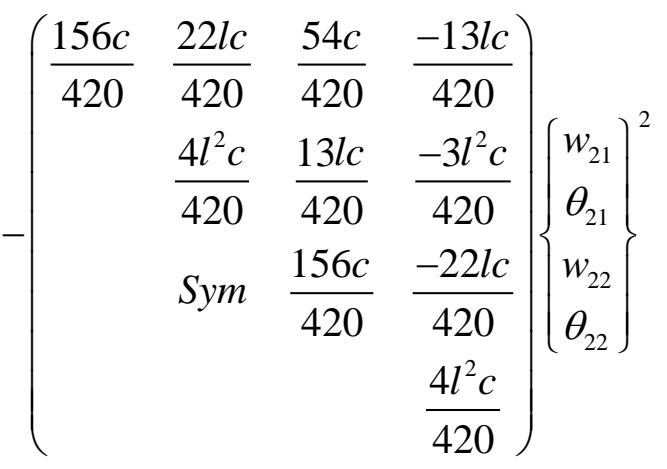


The same also stands valid for mass terms in equations (5.19) - (5.21) of triple-walled carbon nanotubes formulation, whose integral matrices are not presented here for brevity.

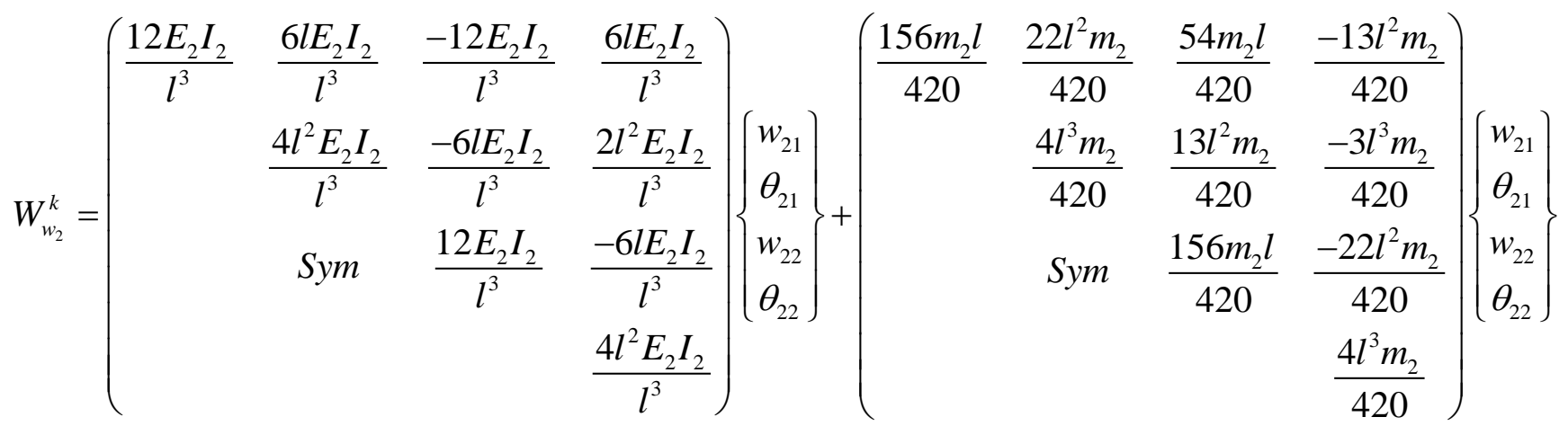

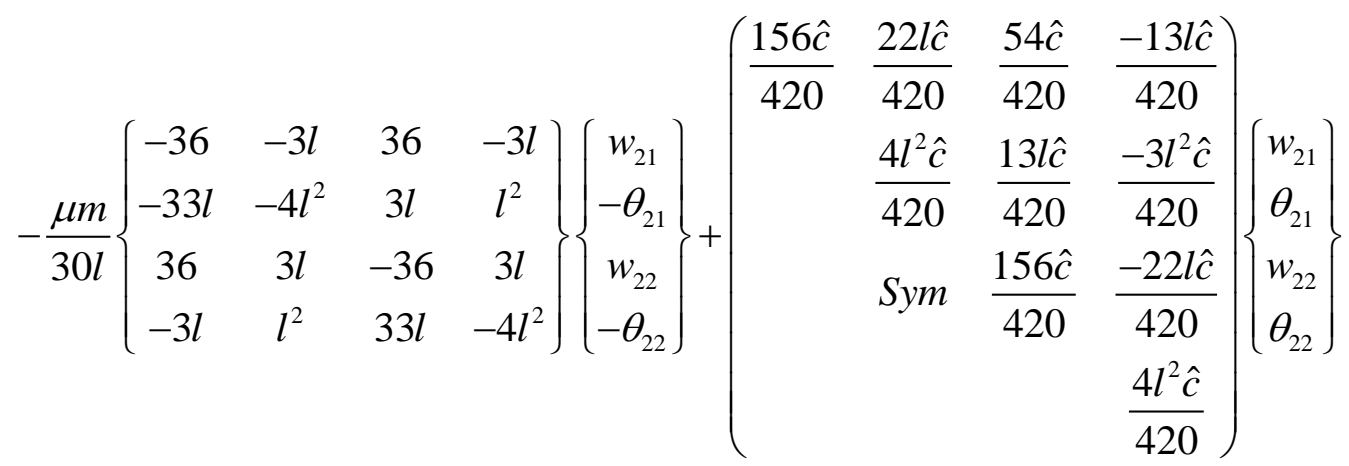

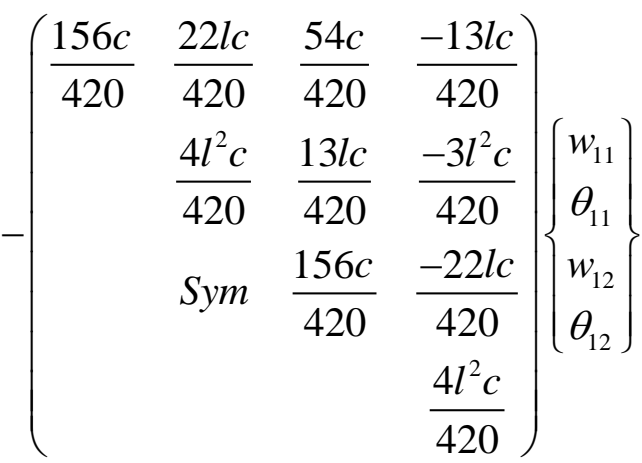

Once again, assembly of element matrices and application of boundary conditions leads to the system's linear eigenvalue problem (6.15), which is then solved to extract the system's natural frequencies and mode.

$$
\operatorname{det}\left(K-\omega^{2} M\right)=0
$$




\subsection{Results and Discussion}

The nonlocal FEM model that is developed in this chapter is demonstrated by using the same geometric and material properties given in [198] with the value of nonlocal parameter $e_{0}=0.82$, is given in equation (6.16) as:

$$
\begin{array}{cc}
L=14 e-9, & \rho_{1}=\rho_{2}=\rho_{3}=2.3 \frac{\mathrm{g}}{\mathrm{cm}^{3}} \\
E_{1}=E_{2}=E_{3}=1 \mathrm{TPa}, & c_{1}=69.43 \mathrm{GPa} ; c_{2}=138.86 \mathrm{GPa} \\
r_{1, i}(\text { inner radius, } \text { inner tube })=0.18 \mathrm{~nm}, & \left.r_{1, o} \text { (outer radius, } \text { inner tube }\right)=0.52 \mathrm{~nm}, \\
r_{2, i}(\text { inner radius, } \text { middle tube })=0.53 \mathrm{~nm}, & \left.r_{2, o} \text { (outer radius, middle tube }\right)=0.87 \mathrm{~nm} \\
\left.r_{3, i} \text { (inner radius, outer tube }\right)=0.88 \mathrm{~nm}, &
\end{array}
$$

\subsubsection{DWCNT modeled as nonlocal Euler-Bernoulli beam}

The natural frequencies of a double-walled CNT, modelled as nonlocal Euler-Bernoulli beam, for different end conditions are given in Table 6.1. The frequency values are compared with the exact values reported by Zhang et al. [56]. It can be seen that the values from the present FEM study are in excellent agreement with the exact values.

As it can be observed in the figures following Table 6.1, the frequency values' convergence is practically achieved even using a course mesh of 10-20 elements, however, the convergence graphs shown in Figs. (6.1) - (6.3) have been produced for up to 50 FEM elements. 
Table 6.1 - FEM natural Frequencies of DWCNTs modeled as nonlocal Euler-Bernoulli beam

\begin{tabular}{|c|c|c|c|c|}
\hline \multicolumn{2}{|c|}{ Boundary Conditions } & $\begin{array}{c}\text { Zhang } \text { et } \mathrm{al} \text {. [56] } \\
\left(10^{12} \mathrm{rad} / \mathrm{s}\right)\end{array}$ & $\begin{array}{c}\text { Present Study } \\
\left(10^{12} \mathrm{rad} / \mathrm{s}\right)\end{array}$ & $\begin{array}{c}\text { Error } \\
\text { Percentage }\end{array}$ \\
\hline \multirow{3}{*}{ Simply Supported } & $\omega_{1}$ & 0.467 & 0.467 & 0.03 \\
\cline { 2 - 5 } & $\omega_{2}$ & 7.885 & 7.885 & 0.00 \\
\hline \multirow{3}{*}{ Clamped-Clamped } & $\omega_{1}$ & 1.056 & 1.057 & 0.08 \\
\cline { 2 - 5 } & $\omega_{2}$ & 7.924 & 7.924 & 0.00 \\
\hline \multirow{3}{*}{ Clamped-Free } & $\omega_{1}$ & 0.166 & 0.166 & 0.02 \\
\cline { 2 - 5 } & $\omega_{2}$ & 7.876 & 7.876 & 0.00 \\
\hline
\end{tabular}

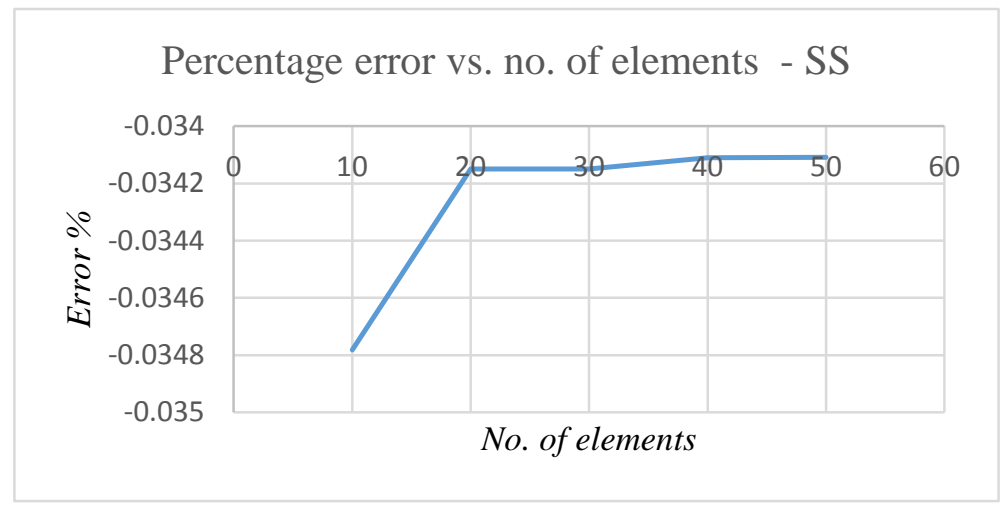

Figure 6.1 - Percentage error vs. the number of elements - SS - Up to 50 FEM elements

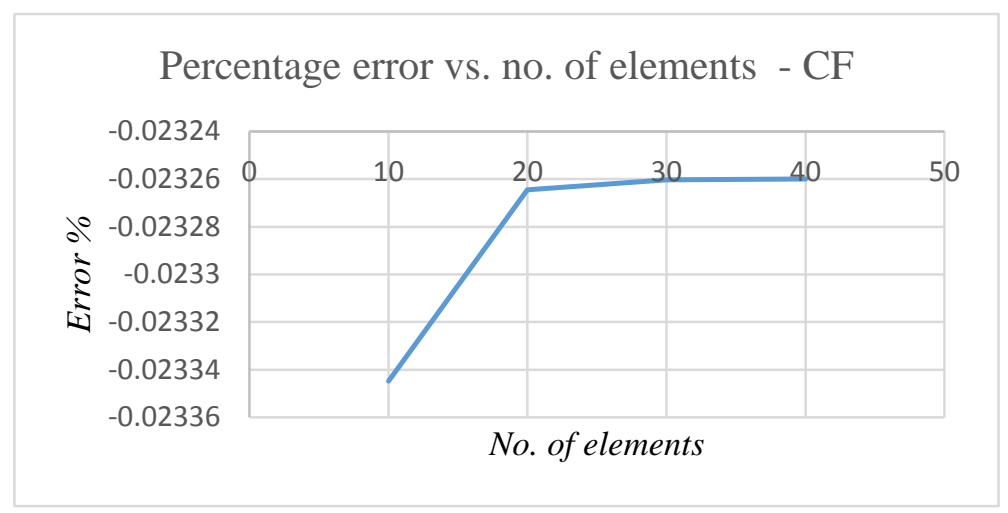

Figure 6.2 - Percentage error vs. the number of elements - CF - Up to 40 FEM elements 


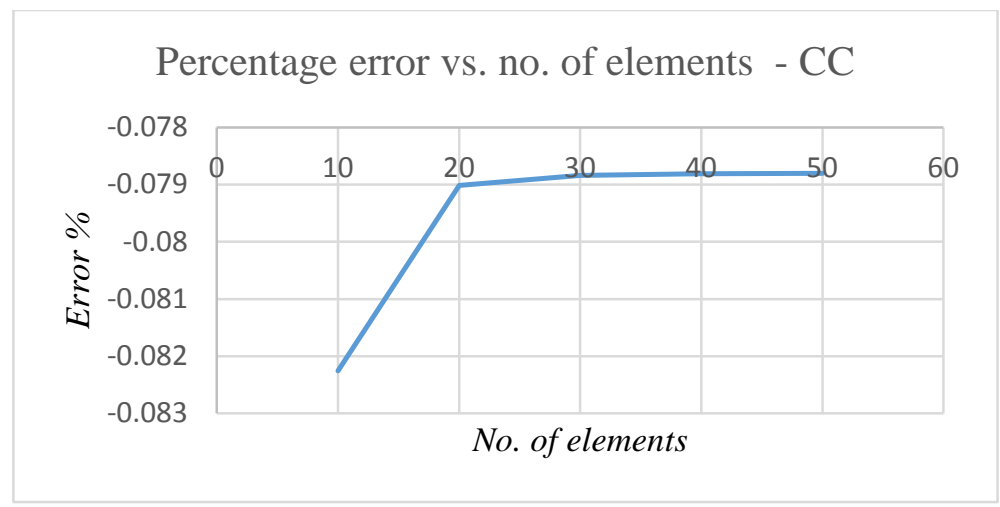

Figure 6.3 - Percentage error vs. the number of elements - CC - Up to 50 FEM elements

The first two mode shapes of double-walled carbon nanotubes, modelled as nonlocal EulerBernoulli beam, and for different boundary conditions, are given in Figures (6.4) - (6.6).

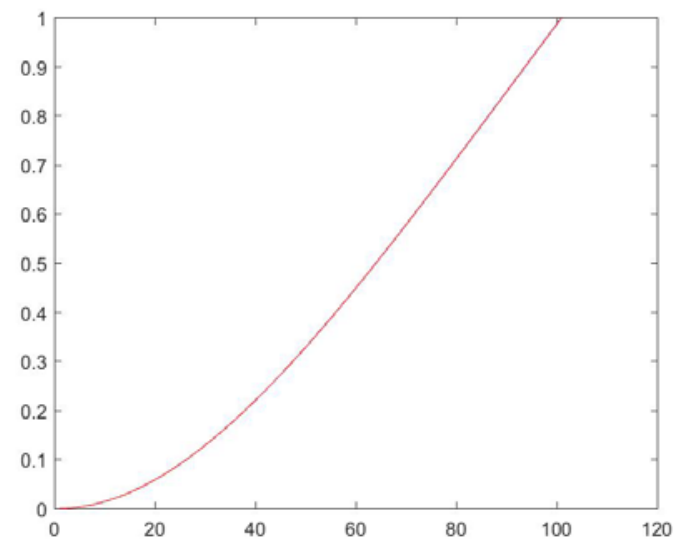

Mode 1

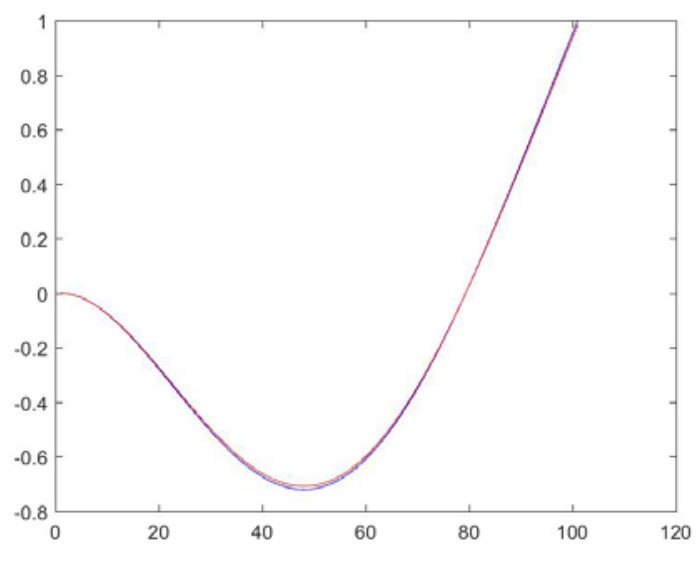

Mode 2

Figure 6.4 - First two mode shapes of DWCNT modeled as nonlocal Euler-Bernoulli - clamped free boundary condition - FEM 


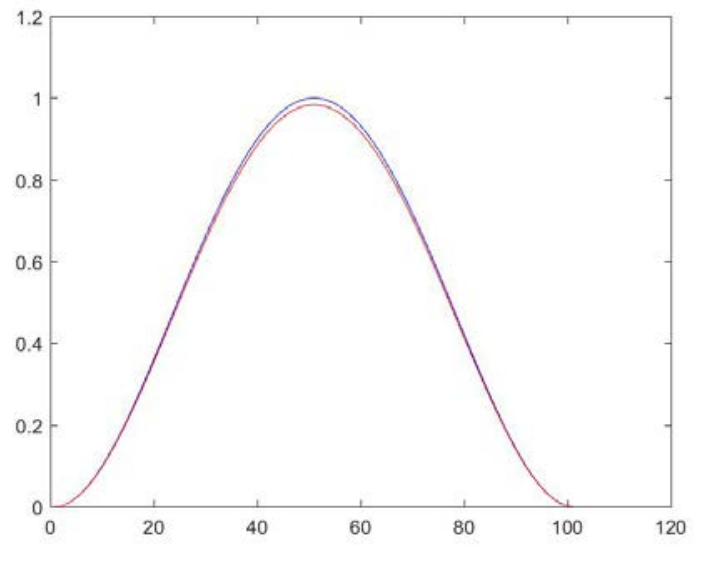

Mode 1

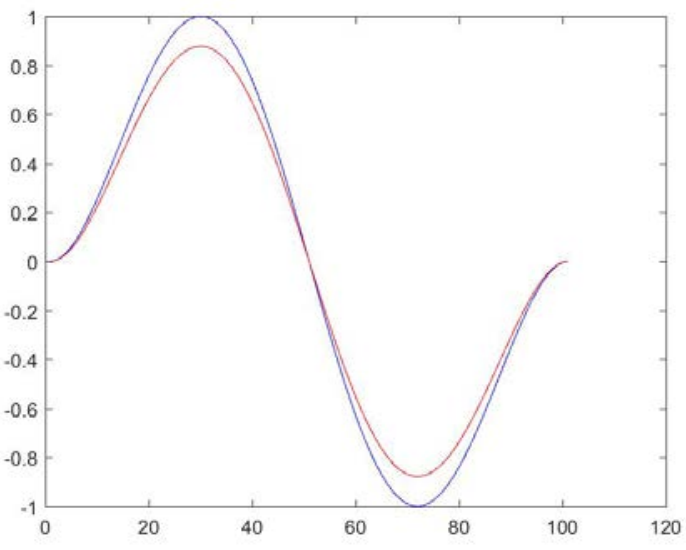

Mode 2

Figure 6.5 - First two mode shapes of DWCNT modeled as nonlocal Euler-Bernoulli - clampedclamped boundary condition - FEM

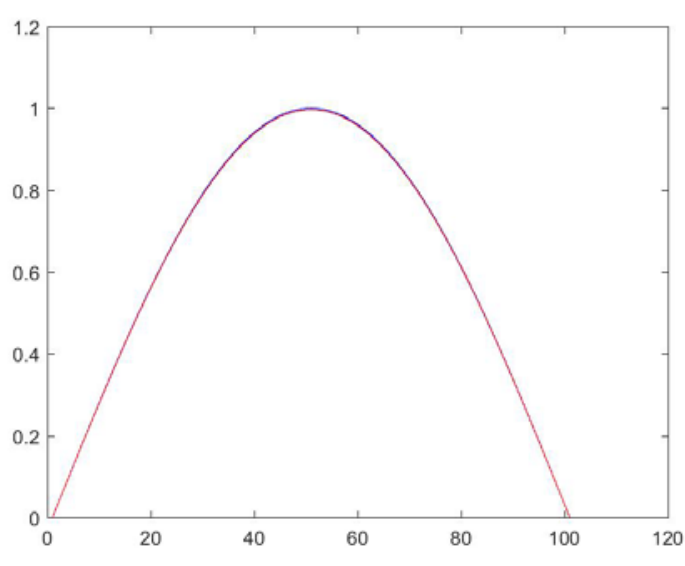

Mode 1

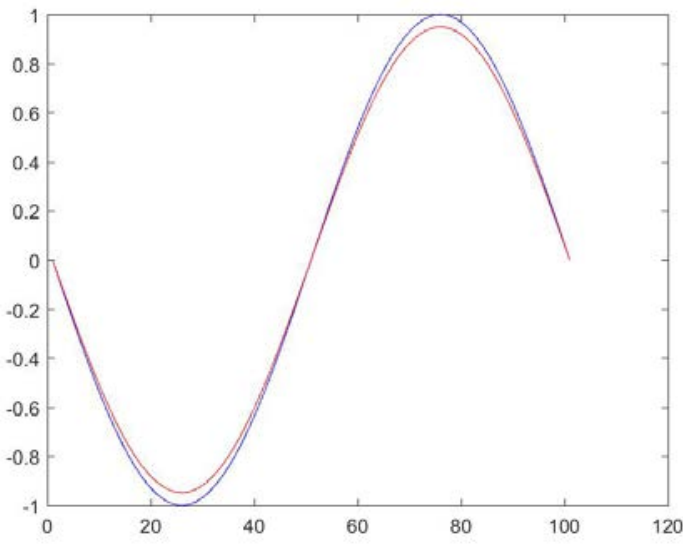

Mode 2

Figure 6.6 - First two mode shapes of DWCNT modeled as nonlocal Euler-Bernoulli - simply supported boundary condition - FEM

\subsubsection{DWCNT modeled as nonlocal Euler-Bernoulli beam embedded in an elastic medium}

For the case of double walled carbon nanotubes, modelled as nonlocal Euler-Bernoulli beam, and embedded in an elastic medium, to the best of author's knowledge no exact values are reported in the open literature. Therefore, the present study relies on convergence of the natural frequencies obtained using the finite element method (FEM) and tabulated in Table 6.2. 
Considering equation (3.8), $\hat{c}=c+k$, where $k$ is the coefficient of the elastic medium surrounding the carbon nanotube, four cases are considered to represent the elastic medium surrounding the carbon nanotubes; i.e., ratio $k / c=100,1,0.1$, and 0.0001 .

Table 6.2 - FEM natural frequencies of DWCNTs modeled as nonlocal Euler-Bernoulli beam embedded in an elastic medium

\begin{tabular}{|c|c|c|c|}
\hline \multirow{3}{*}{ Boundary Conditions } & $k / c$ ratio & $\begin{array}{c}\omega_{11} \\
\left(10^{12} \mathrm{rad} / \mathrm{s}\right)\end{array}$ & $\begin{array}{c}\omega_{12} \\
\left(10^{12} \mathrm{rad} / \mathrm{s}\right)\end{array}$ \\
\hline \multirow{4}{*}{ Simply Supported } & $k / c=100$ & 6.404 & 45.704 \\
\cline { 2 - 4 } & $k / c=1$ & 3.506 & 8.412 \\
\cline { 2 - 4 } & $k / c=0.1$ & 1.256 & 7.929 \\
\cline { 2 - 4 } Clamped-Clamped & $k / c=0.0001$ & 0.468 & 7.885 \\
\hline & $k / c=100$ & 6.431 & 45.717 \\
\cline { 2 - 4 } & $k / c=1$ & 3.613 & 8.458 \\
\cline { 2 - 4 } & $k / c=0.1$ & 1.57 & 7.97 \\
\cline { 2 - 4 } & $k / c=0.0001$ & 1.058 & 7.925 \\
\hline \multirow{5}{*}{ Clamped-Free } & $k / c=100$ & 6.399 & 45.702 \\
\cline { 2 - 4 } & $k / c=1$ & 3.483 & 8.403 \\
\cline { 2 - 4 } & $k / c=0.1$ & 1.179 & 7.921 \\
\cline { 2 - 4 } & $k / c=0.0001$ & 0.17 & 7.877 \\
\hline
\end{tabular}

It can be noticed from Table 6.2 that the non-coaxial natural frequency for the $k / c=100$ is the highest, in the order of the $10^{13}$, owing to the stiffer medium around the carbon nanotube, as it approaches the non-coaxial behavior. It can also be observed that the natural frequency decreases with the decrease in the value of $k / c$, for all boundary conditions. The convergence graphs for $k / c=1$ have been plotted in Figures (6.7) - (6.9), to present the convergence obtained for the values tabulated above. Once again, as it can be observed, the convergence is practically achieved even using a course mesh of 20-30 elements, however, the convergence graphs have been produced for up to $50 \mathrm{FEM}$ elements. 


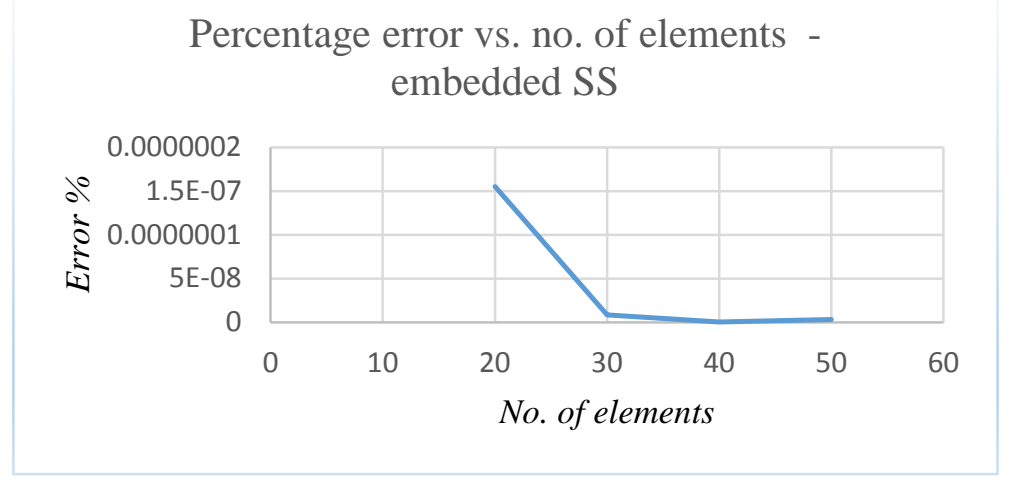

Figure 6.7 - Percentage error vs. the number of elements - embedded SS - Up to 50 FEM elements

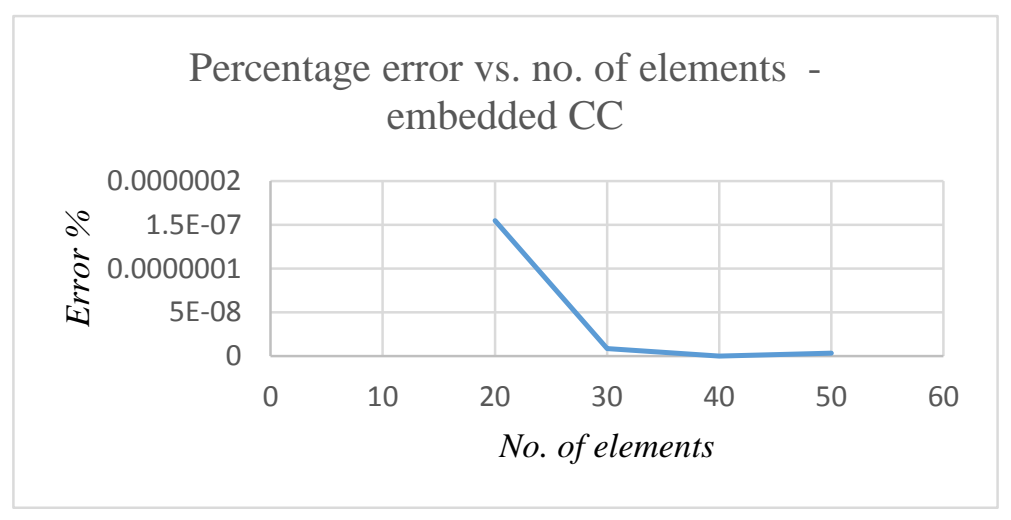

Figure 6.8 - Percentage error vs. the number of elements -embedded CC - Up to 50 FEM elements

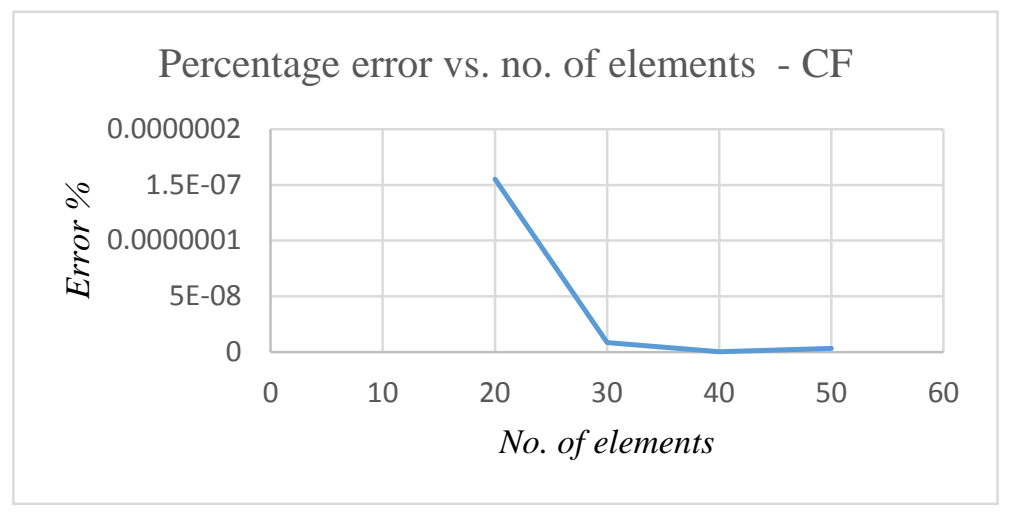

Figure 6.9 - Percentage error vs. the number of elements -embedded CF - Up to 50 FEM elements 
The mode shapes of double-walled CNTs, modelled as nonlocal Euler-Bernoulli beam, and embedded in an elastic medium with $k / c=1$, are given below in Figures (6.10) and (6.11).

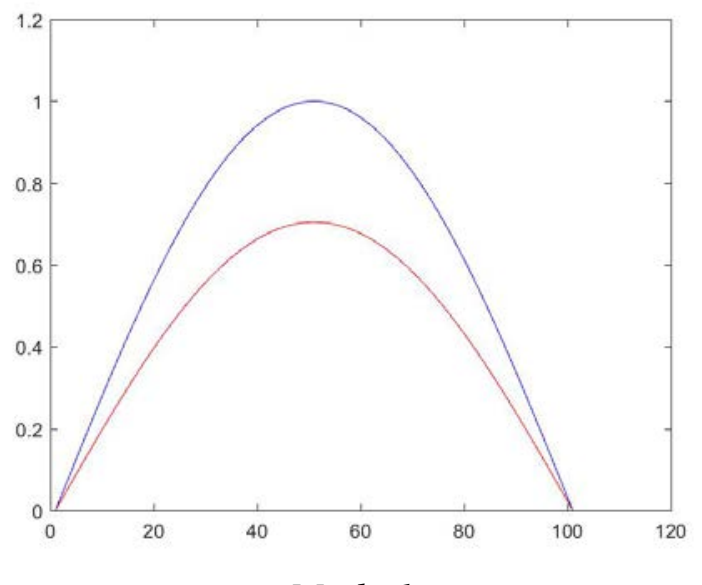

Mode 1

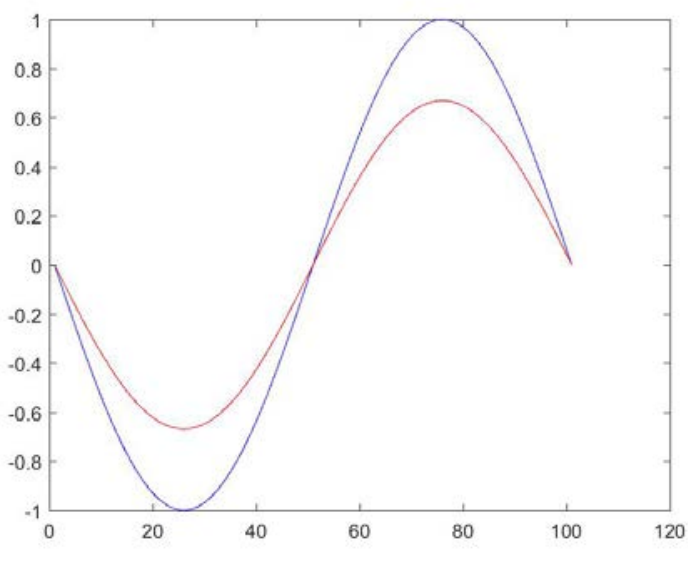

Mode 2

Figure 6.10 - First two mode shapes of DWCNT modeled as nonlocal Euler-Bernoulli, embedded in elastic medium $(k / c=1)$ - simply supported boundary condition - FEM

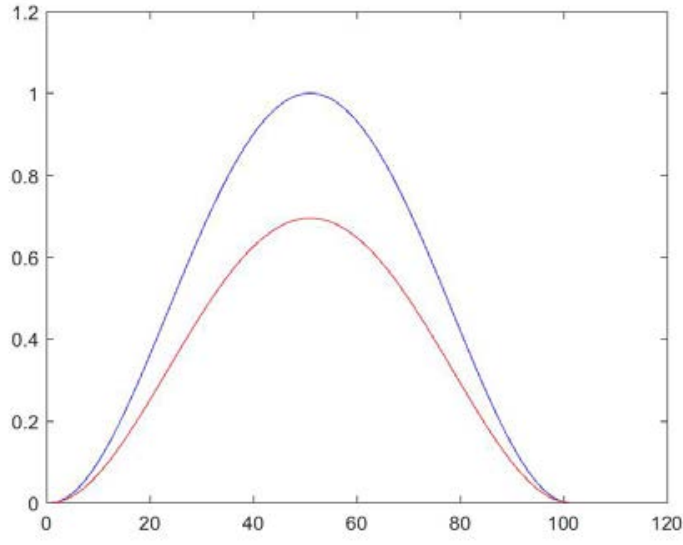

Mode 1

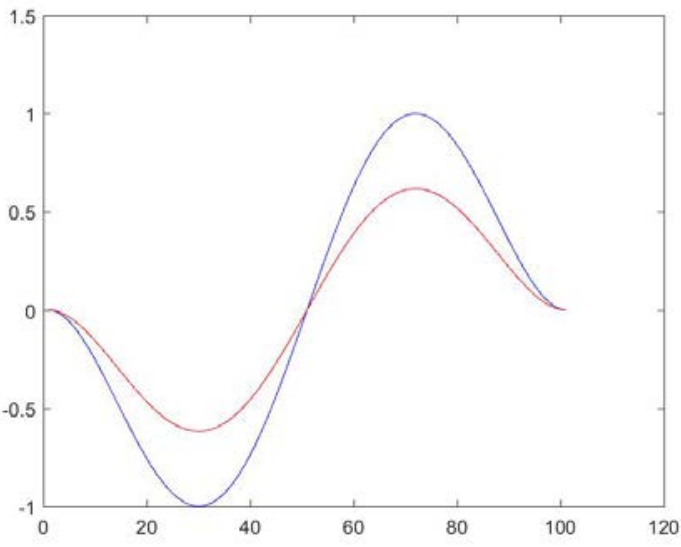

Mode 2

Figure 6.11 - First two mode shapes of DWCNT modeled as nonlocal Euler-Bernoulli, embedded in elastic medium $(\mathrm{k} / \mathrm{c}=1)$ - clamped-clamped boundary condition - FEM 


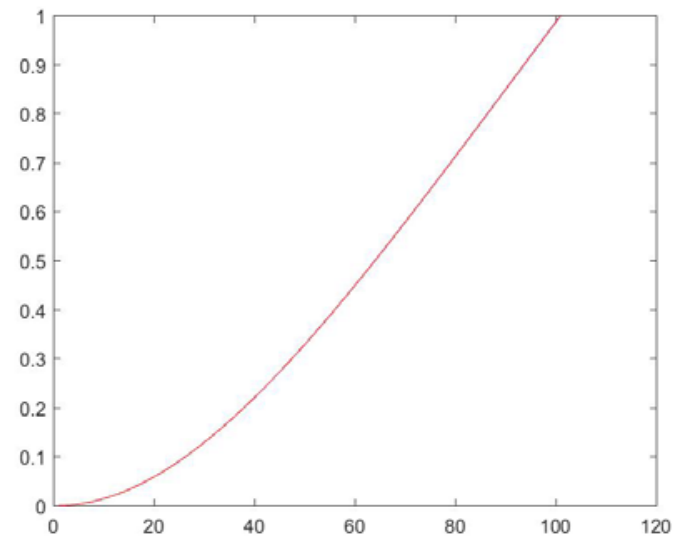

Mode 1

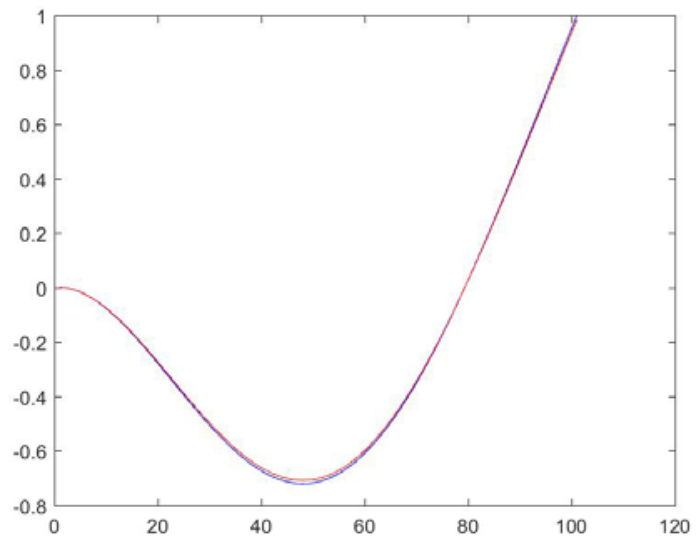

Mode 2

Figure 6.12 - First two mode shapes of DWCNT modeled as nonlocal Euler-Bernoulli, embedded in elastic medium $(k / c=1)$ - clamped-free boundary condition - FEM

\subsubsection{TWCNT modeled as nonlocal Euler-Bernoulli beam}

The natural frequencies of the triple-walled CNTs modelled as nonlocal Euler-Bernoulli beam are shown in Table 6.3, where $\omega_{1}, \omega_{2}$ and $\omega_{3}$ represent the first three natural frequencies of a TWCNT. Once again, as it can be observed, the frequency values' convergence has been practically achieved even using a course mesh of 20-30 elements, however, the convergence graphs shown in Figs. (6.13) to (6.15), for three different boundary conditions and each of TWCNT beams, have been produced for up to 50 FEM elements.

Table 6.3 - FEM natural frequencies of TWCNTs modeled as nonlocal Euler-Bernoulli beam

\begin{tabular}{|c|c|c|c|}
\hline Boundary Conditions & $\begin{array}{c}\omega_{1} \\
\left(10^{12} \mathrm{rad} / \mathrm{s}\right)\end{array}$ & $\begin{array}{c}\omega_{2} \\
\left(10^{12} \mathrm{rad} / \mathrm{s}\right)\end{array}$ & $\begin{array}{c}\omega_{3} \\
\left(10^{12} \mathrm{rad} / \mathrm{s}\right)\end{array}$ \\
\hline Simply Supported & 0.648 & 2.531 & 5.038 \\
\hline Clamped-Clamped & 1.460 & 3.793 & 6.049 \\
\hline Clamped-Free & 0.231 & 1.438 & 3.795 \\
\hline
\end{tabular}




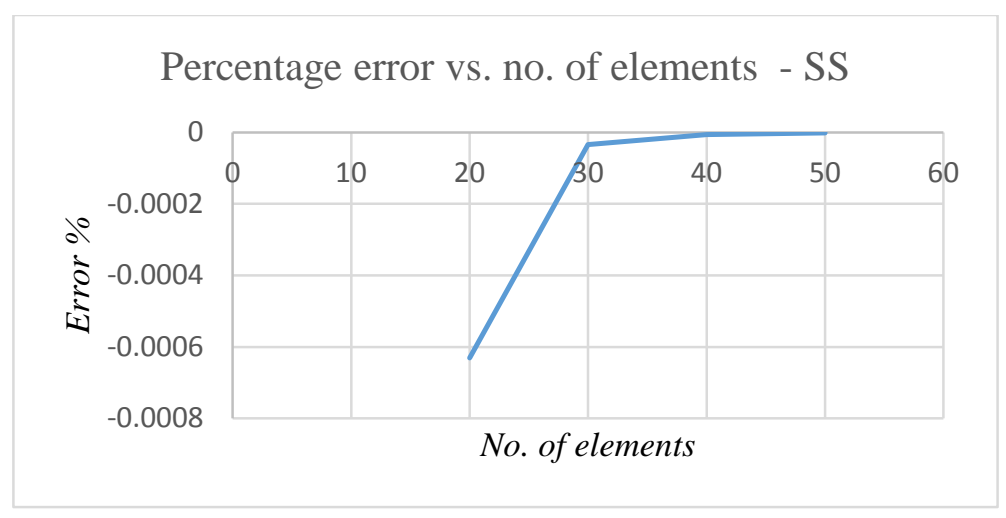

Figure 6.13 - Percentage error vs. the number of elements - SS - Up to 50 FEM elements

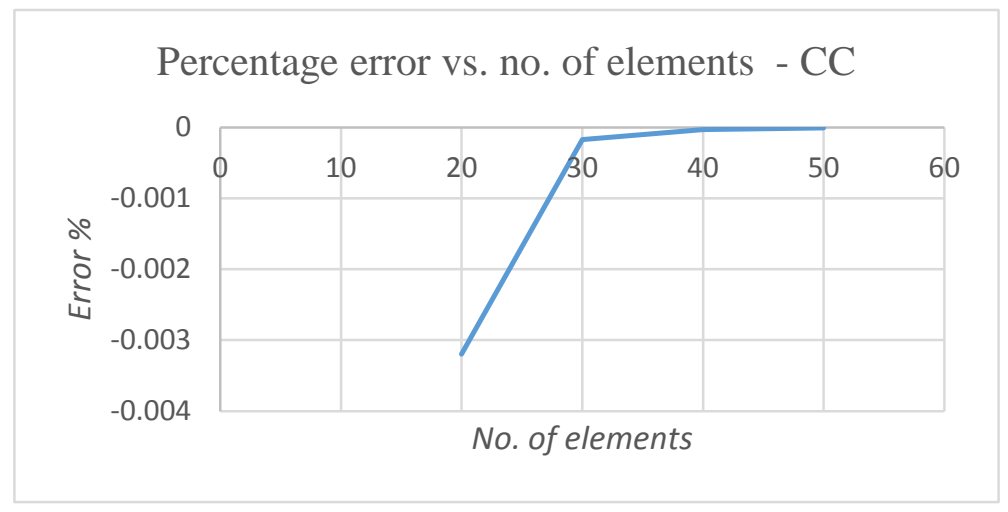

Figure 6.14 - Percentage error vs. the number of elements - CC - Up to 50 FEM elements

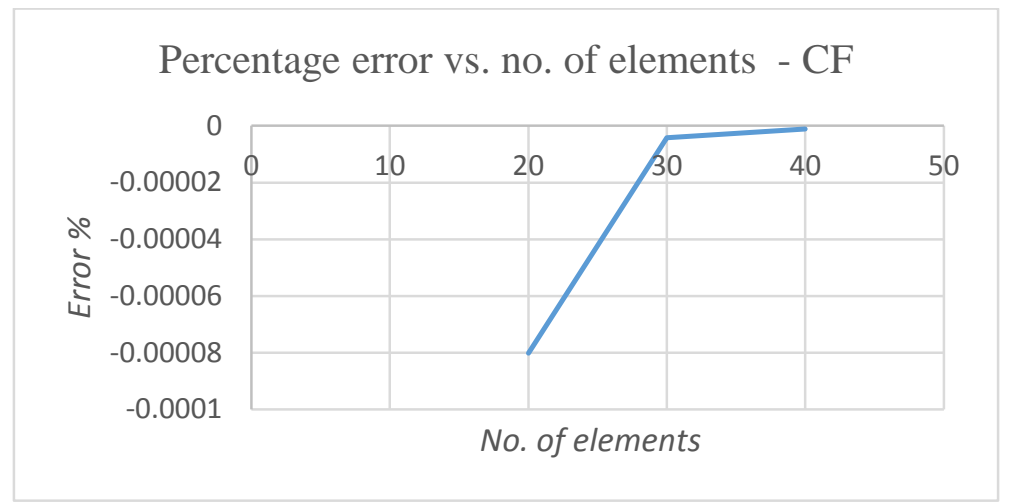

Figure 6.15 - Percentage error vs. the number of elements - CF - Up to 40 FEM elements

The mode shapes of a TWCNT modeled as nonlocal Euler-Bernoulli for different boundary conditions are presented in Figures (6.16) - (6.18) 


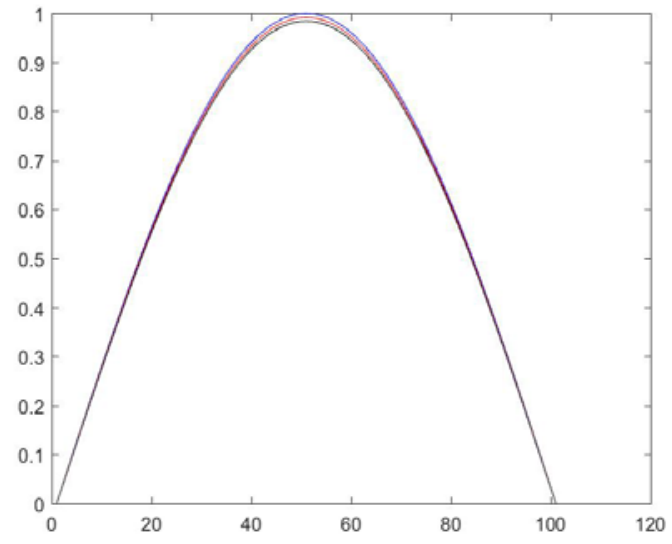

Mode 1

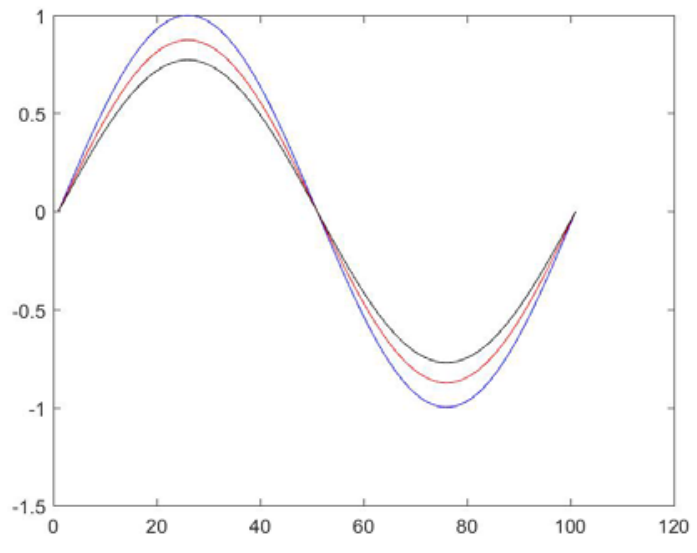

Mode 2

Figure 6.16 - First two mode shapes of TWCNT modeled as nonlocal Euler-Bernoulli - simply supported boundary condition - FEM

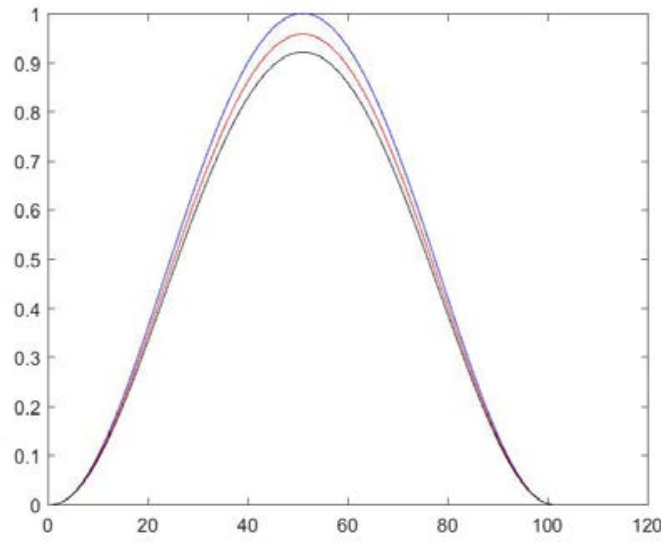

Mode 1

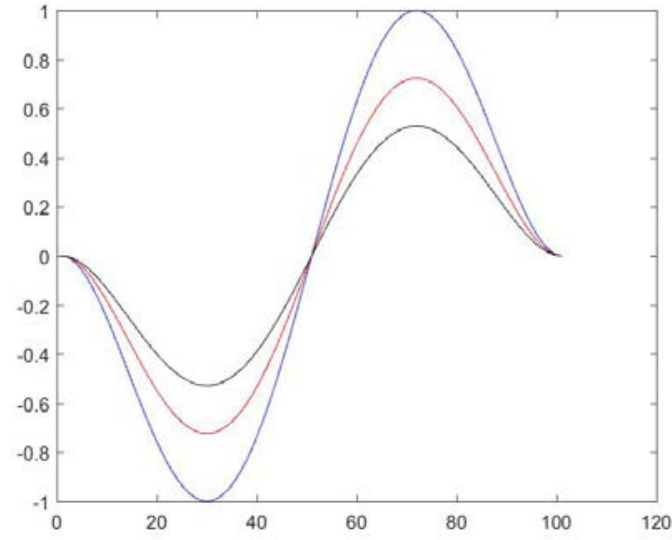

Mode 2

Figure 6.17 - First two mode shapes of TWCNT modeled as nonlocal Euler-Bernoulli - clampedclamped boundary condition - FEM 

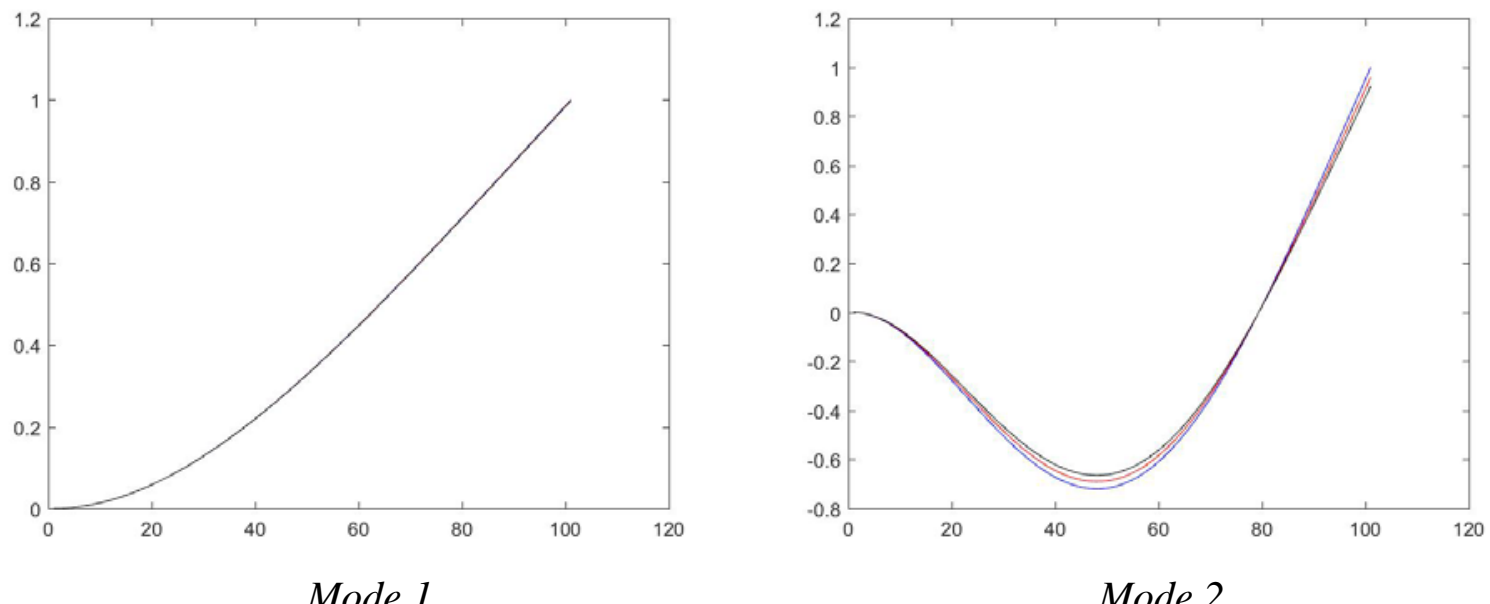

Figure 6.18 - First two mode shapes of TWCNT modeled as nonlocal Euler-Bernoulli-clampedfree boundary condition

\subsubsection{TWCNT modeled as nonlocal Euler-Bernoulli beam embedded in an elastic medium}

As is done for the entire study, four cases will be considered to represent the coefficient of elastic medium surrounding the TWCNTs. Equation (3.8), i.e., $\hat{c}=c+k$, is taken and the converged natural frequencies for four $k / c$ ratios are tabulated in Table 6.4.

Table 6.4 - FEM natural frequencies of TWCNTs modeled as nonlocal Euler-Bernoulli beam embedded in an elastic medium

\begin{tabular}{|c|c|c|c|c|}
\hline $\begin{array}{l}\text { Boundary } \\
\text { Conditions }\end{array}$ & $k$ & $\begin{array}{c}\omega_{1} \\
\left(10^{12} \mathrm{rad} / \mathrm{s}\right)\end{array}$ & $\begin{array}{c}\omega_{2} \\
\left(10^{12} \mathrm{rad} / \mathrm{s}\right)\end{array}$ & $\begin{array}{c}\omega_{3} \\
\left(10^{12} \mathrm{rad} / \mathrm{s}\right)\end{array}$ \\
\hline \multirow{4}{*}{$\begin{array}{c}\text { Simply } \\
\text { Supported }\end{array}$} & $k / c=100$ & 4.494 & 4.737 & 5.606 \\
\hline & $k / c=1$ & 3.138 & 3.736 & 5.260 \\
\hline & $k / c=0.1$ & 1.307 & 2.734 & 5.070 \\
\hline & $k / c=0.0001$ & 0.648 & 2.531 & 5.038 \\
\hline \multirow{4}{*}{$\begin{array}{l}\text { Clamped- } \\
\text { Clamped }\end{array}$} & $k / c=100$ & 4.563 & 5.074 & 6.323 \\
\hline & $k / c=1$ & 3.321 & 4.413 & 6.178 \\
\hline & $k / c=0.1$ & 1.833 & 3.890 & 6.129 \\
\hline & $k / c=0.0001$ & 1.460 & 3.793 & 6.048 \\
\hline \multirow{4}{*}{ Clamped-Free } & $k / c=100$ & 4.480 & 4.561 & 5.074 \\
\hline & $k / c=1$ & 3.098 & 3.314 & 4.414 \\
\hline & $k / c=0.1$ & 1.164 & 1.817 & 3.891 \\
\hline & $k / c=0.0001$ & 0.234 & 1.438 & 3.794 \\
\hline
\end{tabular}


The following Figures (6.19) - (6.21) have been plotted for the $k / c=1$ to present the convergence of the natural frequencies reported. Once again, as it can be observed, the frequency values’ convergence has been practically achieved even using a course mesh of 20-30 elements, however, the convergence graphs shown in Figs. (6.19) to (6.21), for three different boundary conditions and each of TWCNT beams, have been produced for up to 50 FEM elements.

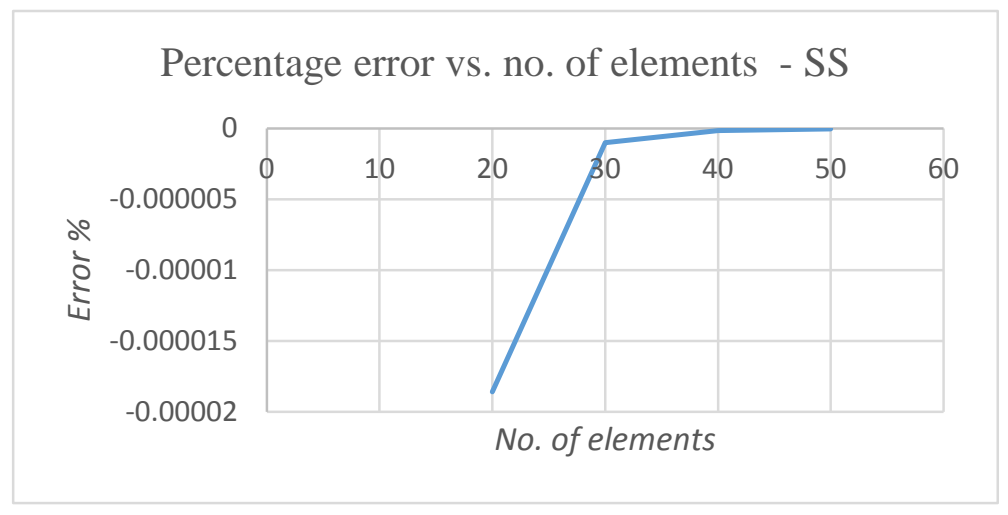

Figure 6.19 - Percentage error vs. the number of elements -embedded SS TWCNT - 50 FEM elements

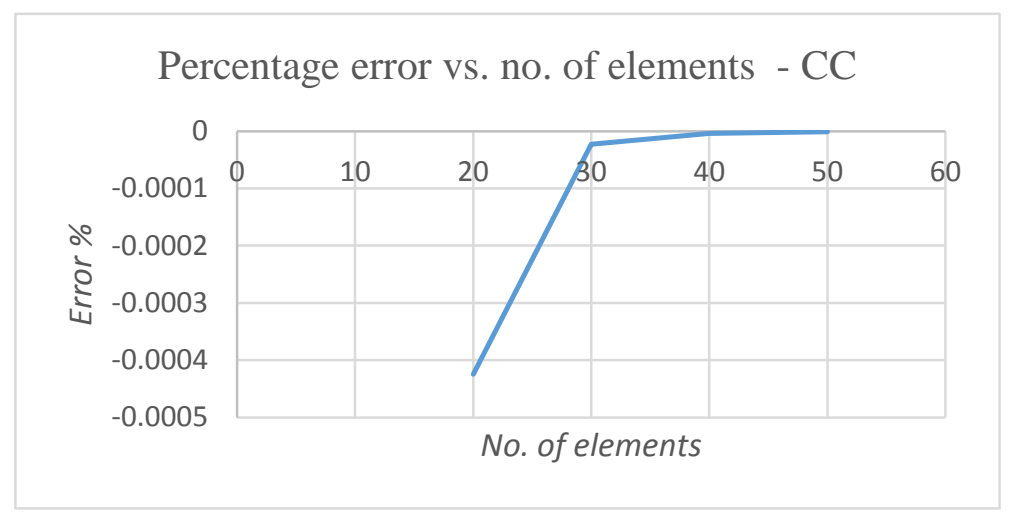

Figure 6.20 - Percentage error vs. the number of elements -embedded CC TWCNT - 50 FEM elements 


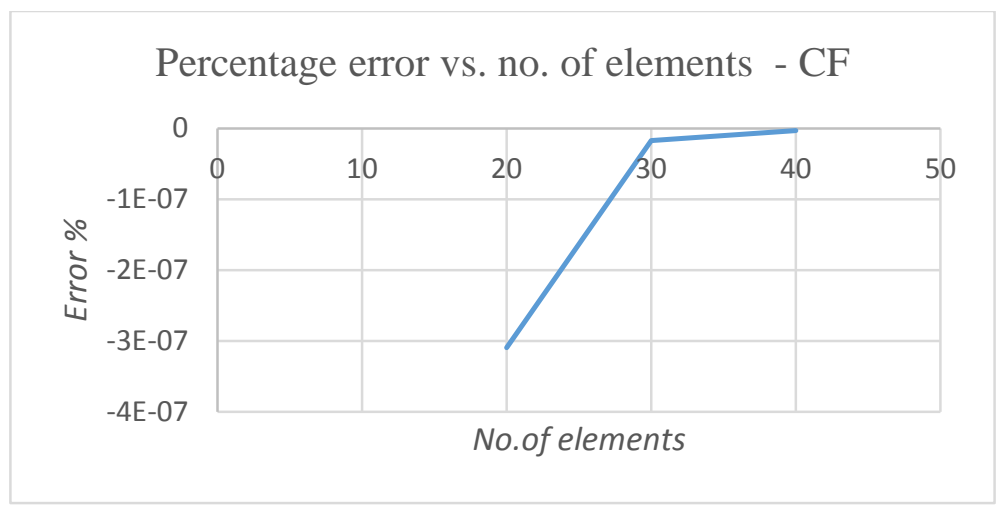

Figure 6.21 - Percentage error vs. the number of elements -embedded CF TWCNT - 50 FEM elements

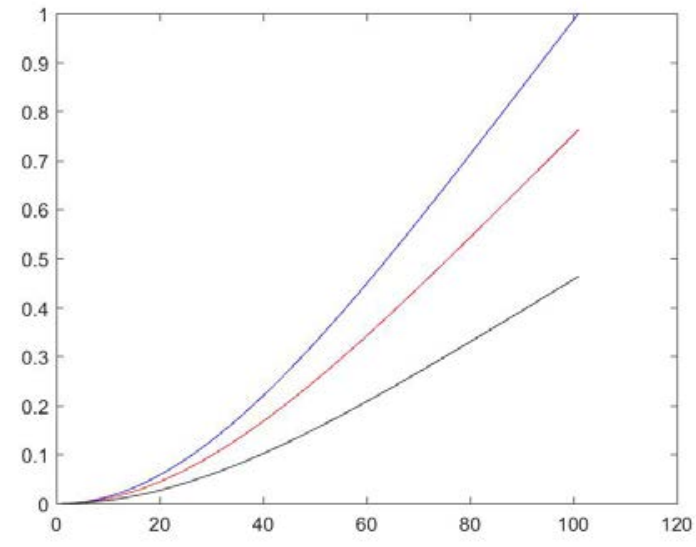

Mode 1

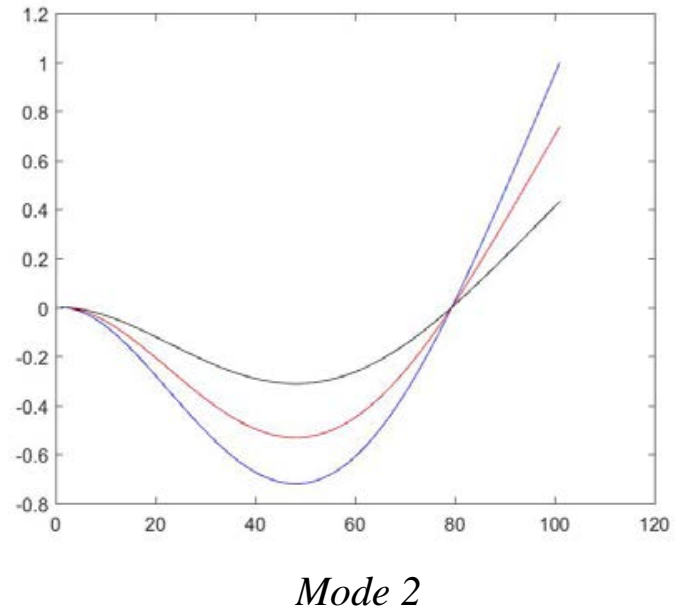

Mode 2

Figure 6.22 - First two mode shapes of TWCNT modeled as nonlocal Euler-Bernoulli, embedded in an elastic medium - clamped-free boundary condition - FEM 


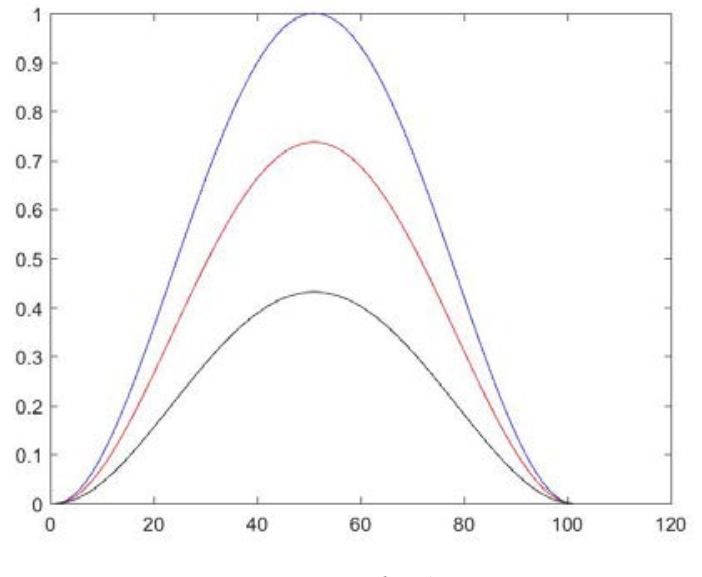

Mode 1

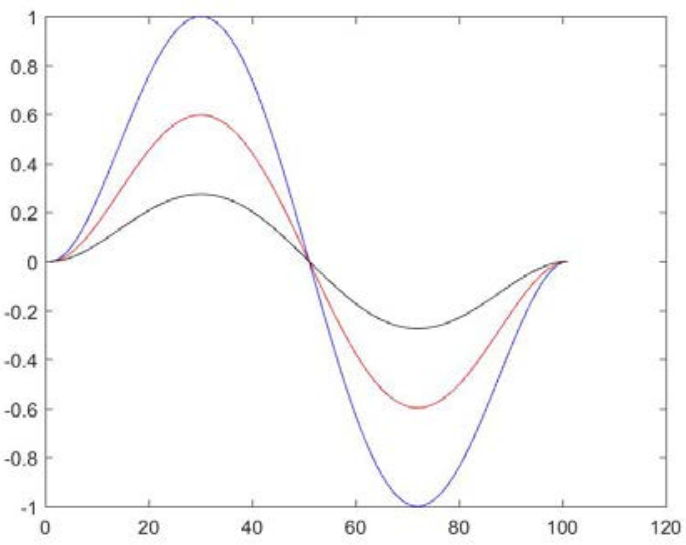

Mode 2

Figure 6.23 - First two mode shapes of TWCNT modeled as nonlocal Euler-Bernoulli, embedded in an elastic medium - clamped-clamped boundary condition - FEM

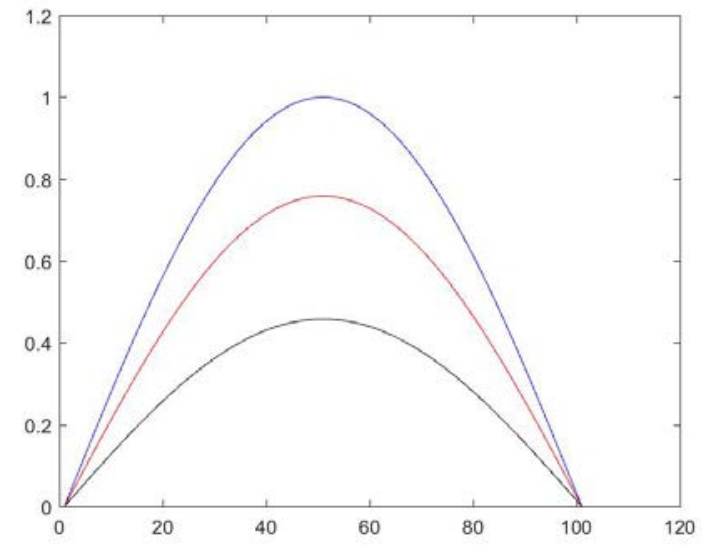

Mode 1

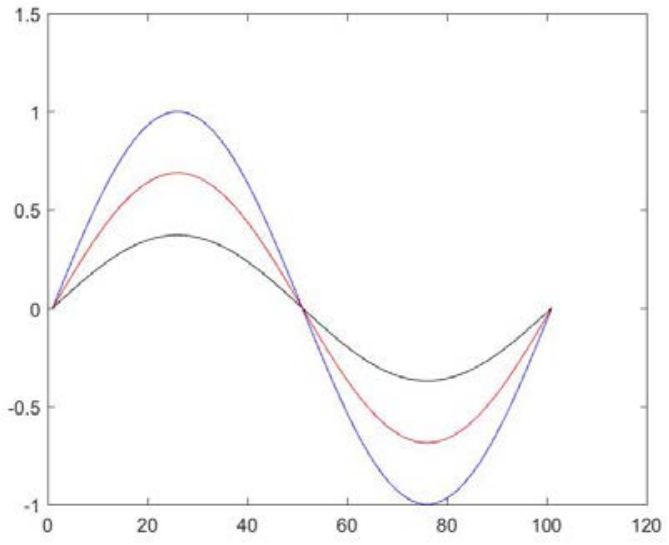

Mode 2

Figure 6.24 - First two mode shapes of TWCNT modeled as nonlocal Euler-Bernoulli, embedded in an elastic medium - simply supported boundary condition - FEM

\subsection{Conclusion}

In this chapter, finite element method (FEM) formulation was developed for the vibration analysis of double- and triple-walled carbon nanotubes, with and without surrounding elastic medium of different stiffness values, modelled as nonlocal Euler-Bernoulli beam. The developed FEM was then applied illustrative DWCNT and TWCNT examples and the systems' natural 
frequencies are found. The frequency data were then compared with the values available in the open literature, where applicable, and are found to be in agreement. It is worth noting that the error percentage between natural frequencies of local and nonlocal Euler-Bernoulli beams is negligible, which can be attributed to the fact that this study considers the $L / d$ ratio equal to 10 and is possible that the effect of nonlocal parameter on the natural frequency becomes more prominent for higher $L / d$ ratios, but would require further investigation. Due to the lack of published data in some cases, the convergence of frequency values have been established as a means of verification of correctness of formulation and results. 


\section{Conclusions and Future Work}

Since their discovery, immense attention has been given to carbon nanotubes, due to their exceptional thermal, electronic and mechanical properties. The presented study in this thesis employs finite element method (FEM) formulation to study the free vibrations of double and triple walled carbon nanotubes, modelled as Euler-Bernoulli beams. The multiple-beam model employed assumes van der Waals forces acting as the coupling factor between the tubes. Galerkin weighted residual approach is used to derive the mass and stiffness matrices and the resulting linear eigenvalue problem is solved to extract the system's natural frequencies and mode shapes. The frequency results are compared to, and are found to be in agreement with, values reported in the open literature. An analytical formulation, known as the Dynamic Stiffness Matrix (DSM), for the free vibration analysis of double-walled carbon nanotubes (DWCNTs) is also developed and presented for the first time. The DSM formulation revolves around the use of extensive mathematical and matrix operations carried out on the closed form solutions to a single higher- (eighth, in this case) order differential equation derived by combining the coupled (two fourth order, in this case) governing equations. The resulting Dynamic (frequency-dependent) stiffness matrix leads to a nonlinear eigenvalue problem, which is then solved using Wittrick-Williams (W-W) root counting algorithm to find the system's natural frequencies and modes.

Finite element formulation is also applied to the dynamic analysis of flexible carbon nanotubes (CNTs) modelled as nonlocal Euler-Bernoulli beams, which takes into account the C$\mathrm{C}$ bond length to bridge the gap between theoretical and experimental field of carbon nanotubes. All the cases studied in the thesis also considered the case where carbon nanotubes are embedded in elastic medium, thereby paving way for the development of analytical and/or better numerical methods to study of CNT-based nanocomposites. The study confirms that both the numerical (FEM) and exact/analytical (DSM) methods, generally used for continuum structures, perform well and could also be exploited to further investigate and understand the vibrational behavior of CNTs. 
Contributions of this thesis would include explicit FEM formulation of DWCNTs and TWCNTs using local and nonlocal Euler-Bernoulli beams and DSM formulation of DWCNT using local Euler-Bernoulli beam.

For future research, the exactness of the Dynamic Stiffness Matrix (DSM) can be combined with the generality of the finite element formulation using trigonometric shape functions, as suggested by Dynamic Finite Element (DFE) approach. Similar methods could also be employed to study the buckling loads for carbon nanotubes. Further, ropes and wires of carbon nanotubes can be studied by assuming the van der Waals forces acting uniformly between all the carbon nanotubes in the rope. Similar studies can be carried out using other continuum structures, with the aim of arriving at a model that could robustly account for all the parameters of the carbon nanotubes vibration, taking into account the chirality and other small scale effects. These studies could be further extended to two-dimensions so as to study graphene sheets. A comprehensive program could be developed that would snapshot the vibrational behavior of the carbon nanotube by assuming it as any continuum structure, based on the application it is to be used in, and the forces that would come into play. 


\section{References}

[1] “Global Markets and Technologies for Carbon Nanotubes,” 2015.

[2] C. Li and T.-W. Chou, "A structural mechanics approach for the analysis of carbon nanotubes,” International Journal of Solids and Structures, vol. 40, no. 10, pp. 24872499, 2003.

[3] B. I. Yakobson and R. E. Smalley, “Fullerene nanotubes: C 1,000,000 and beyond: Some unusual new molecules-long, hollow fibers with tantalizing electronic and mechanical properties - have joined diamonds and graphite in the carbon family,” American Scientist, pp. 324-337, 1997.

[4] G. M. Odegard, T. S. Gates, L. M. Nicholson, and K. E. Wise, "Equivalent-continuum modelling of nano-structured materials,” Composites Science and Technology, vol. 62, no. 14, pp. 1869-1880, 2002.

[5] C. Li and T.-W. Chou, "Modelling of elastic buckling of carbon nanotubes by molecular structural mechanics approach,” Mechanics of Materials, vol. 36, no. 11, pp. 1047-1055, 2004.

[6] C. Li and T.-W. Chou, "Single-walled carbon nanotubes as ultrahigh frequency nanomechanical resonators,” Physical review B, vol. 68, no. 7, p. 073405, 2003.

[7] C. Li and T.-W. Chou, "Vibrational behaviors of multiwall-carbon-nanotube-based nanomechanical resonators,” Applied Physics Letters, vol. 84, no. 1, pp. 121-123, 2004.

[8] C. Li and T.-W. Chou, "Atomistic modelling of carbon nanotube-based mechanical sensors,” Journal of intelligent material systems and structures, vol. 17, no. 3, pp. 247254, 2006.

[9] K. Chandraseker, S. Mukherjee, J. T. Paci, and G. C. Schatz, “An atomistic-continuum Cosserat rod model of carbon nanotubes," Journal of the Mechanics and Physics of Solids, vol. 57, no. 6, pp. 932-958, 2009.

[10] S. Hollerer and C. C. Celigoj, "Buckling analysis of carbon nanotubes by a mixed atomistic and continuum model,” Computational Mechanics, vol. 51, no. 5, pp. 765-789, 2013.

[11] Y.-G. Hu, K. Liew, and Q. Wang, "Modelling of vibrations of carbon nanotubes,” Procedia Engineering, vol. 31, pp. 343-347, 2012.

[12] P. R. Budarapu, S. S. Yb, B. Javvaji, and D. R. Mahapatra, "Vibration analysis of multiwalled carbon nanotubes embedded in elastic medium," Frontiers of Structural and Civil Engineering, vol. 8, no. 2, pp. 151-159, 2014. 
[13] R. Artan and L. Lehmann, "The vibrations of carbon nanotubes in nonlocal elasticity," Journal of Computational and Theoretical Nanoscience, vol. 6, no. 3, pp. 653-661, 2009.

[14] C. Ru, "Effect of van der Waals forces on axial buckling of a double-walled carbon nanotube,” Journal of Applied Physics, vol. 87, no. 10, pp. 7227-7231, 2000.

[15] C. Wang, C. Ru, and A. Mioduchowski, "Applicability and limitations of simplified elastic shell equations for carbon nanotubes," Journal of applied mechanics, vol. 71, no. 5, pp. 622-631, 2004.

[16] C. Wang, C. Ru, and A. Mioduchowski, "Free vibration of multiwall carbon nanotubes," Journal of Applied Physics, vol. 97, no. 11, p. 114323, 2005.

[17] R. Ansari, S. Rouhi, and M. Aryayi, "Nanoscale finite element models for vibrations of single-walled carbon nanotubes: atomistic versus continuum," Applied Mathematics and Mechanics, vol. 34, no. 10, pp. 1187-1200, 2013.

[18] A. Ghorbanpour Arani, M. Mohammadimehr, A. Arefmanesh, and A. Ghasemi, "Transverse vibration of short carbon nanotubes using cylindrical shell and beam models," Proceedings of the Institution of Mechanical Engineers, Part C: Journal of Mechanical Engineering Science, vol. 224, no. 3, pp. 745-756, 2010.

[19] A. Pantano, D. M. Parks, and M. C. Boyce, "Mechanics of deformation of single-and multi-wall carbon nanotubes," Journal of the Mechanics and Physics of Solids, vol. 52, no. 4, pp. 789-821, 2004.

[20] C. Wang, C. Ru, and A. Mioduchowski, "Buckling and free vibration of multiwall carbon nanotubes,” AIP Publishing, 2005.

[21] T. Natsuki, M. Endo, and Q. Q. Ni, "Vibrational analysis of fluid-filled carbon nanotubes using the wave propagation approach,” Applied Physics A, vol. 90, no. 3, pp. 441-445, 2008 .

[22] A. Tylikowski, "Instability of thermally induced vibrations of carbon nanotubes," Archive of Applied Mechanics, vol. 78, no. 1, pp. 49-60, 2008.

[23] A. C. Eringen and D. Edelen, “On nonlocal elasticity,” International Journal of Engineering Science, vol. 10, no. 3, pp. 233-248, 1972.

[24] A. C. Eringen, "Nonlocal polar elastic continua," International Journal of Engineering Science, vol. 10, no. 1, pp. 1-16, 1972.

[25] A. C. Eringen, "On differential equations of nonlocal elasticity and solutions of screw dislocation and surface waves," Journal of Applied Physics, vol. 54, no. 9, pp. 4703-4710, 1983. 
[26] J. Peddieson, G. R. Buchanan, and R. P. McNitt, "Application of nonlocal continuum models to nanotechnology," International Journal of Engineering Science, vol. 41, no. 3, pp. 305-312, 2003.

[27] Y. Zhang, G. Liu, and J. Wang, "Small-scale effects on buckling of multiwall carbon nanotubes under axial compression,” Physical review B, vol. 70, no. 20, p. 205430, 2004.

[28] Q. Wang and C. Wang, "The constitutive relation and small scale parameter of nonlocal continuum mechanics for modelling carbon nanotubes," Nanotechnology, vol. 18, no. 7, p. 075702, 2007.

[29] Y.-G. Hu, K. M. Liew, Q. Wang, X. He, and B. Yakobson, "Nonlocal shell model for elastic wave propagation in single-and double-walled carbon nanotubes," Journal of the Mechanics and Physics of Solids, vol. 56, no. 12, pp. 3475-3485, 2008.

[30] Q. Wang and V. Varadan, "Application of nonlocal elastic shell theory in wave propagation analysis of carbon nanotubes," Smart Materials and Structures, vol. 16, no. 1, p. 178, 2007.

[31] F. Khademolhosseini, R. Rajapakse, and A. Nojeh, "Torsional buckling of carbon nanotubes based on nonlocal elasticity shell models," Computational Materials Science, vol. 48, no. 4, pp. 736-742, 2010.

[32] F. Khademolhosseini, A. S. Phani, A. Nojeh, and N. Rajapakse, "Nonlocal continuum modelling and molecular dynamics simulation of torsional vibration of carbon nanotubes,” Nanotechnology, IEEE Transactions on, vol. 11, no. 1, pp. 34-43, 2012.

[33] G. Mikhasev, "On localized modes of free vibrations of single-walled carbon nanotubes embedded in nonhomogeneous elastic medium," ZAMM-Journal of Applied Mathematics and Mechanics/Zeitschrift für Angewandte Mathematik und Mechanik, vol. 94, no. 1-2, pp. 130-141, 2014.

[34] R. Ansari, H. Rouhi, and S. Sahmani, "Free vibration analysis of single-and double-walled carbon nanotubes based on nonlocal elastic shell models," Journal of Vibration and Control, vol. 20, no. 5, pp. 670-678, 2014.

[35] Q. Deng and Z. Yang, "Vibration of fluid-filled multi-walled carbon nanotubes seen via nonlocal elasticity theory," Acta Mechanica Solida Sinica, vol. 27, no. 6, pp. 568-578, 2014.

[36] M. Shaban and A. Alibeigloo, "Three dimensional vibration and bending analysis of carbon nanotubes embedded in elastic medium based on theory of elasticity," Latin American Journal of Solids and Structures, vol. 11, no. 12, pp. 2122-2140, 2014. 
[37] C. Ru, "Column buckling of multiwall carbon nanotubes with interlayer radial displacements,” Physical Review B, vol. 62, no. 24, p. 16962, 2000.

[38] C. Ru, "Elastic models for carbon nanotubes," in Encyclopedia of nanoscience and nanotechnology, vol. 2, no. 744, American Scientific Publishers, 2004, pp. 731-744.

[39] J. Yoon, “Elastic Beam Models for Dynamics of Multiwall Carbon Nanotubes,” 2006.

[40] Y. Fu, J. Hong, and X. Wang, “Analysis of nonlinear vibration for embedded carbon nanotubes,” Journal of Sound and Vibration, vol. 296, no. 4, pp. 746-756, 2006.

[41] J. Yoon, C. Ru, and A. Mioduchowski, "Vibration of an embedded multiwall carbon nanotube,” Composites Science and Technology, vol. 63, no. 11, pp. 1533-1542, 2003.

[42] T. Natsuki, Q.-Q. Ni, and M. Endo, “Analysis of the vibration characteristics of doublewalled carbon nanotubes,” Carbon, vol. 46, no. 12, pp. 1570-1573, 2008.

[43] T. Natsuki, X.-W. Lei, Q.-Q. Ni, and M. Endo, "Free vibration characteristics of doublewalled carbon nanotubes embedded in an elastic medium,” Physics Letters A, vol. 374, no. 26, pp. 2670-2674, 2010.

[44] T. Natsuki, X.-W. Lei, Q.-Q. Ni, and M. Endo, "Vibrational analysis of double-walled carbon nanotubes with inner and outer nanotubes of different lengths," Physics Letters A, vol. 374, no. 46, pp. 4684-4689, 2010.

[45] C. Wang, V. Tan, and Y. Zhang, "Timoshenko beam model for vibration analysis of multi-walled carbon nanotubes,” Journal of Sound and Vibration, vol. 294, no. 4, pp. 1060-1072, 2006.

[46] C. Wang, Y. Zhang, Y. Xiang, and J. Reddy, "Recent studies on buckling of carbon nanotubes,” Applied Mechanics Reviews, vol. 63, no. 3, p. 030804, 2010.

[47] H. Shima, "Buckling of carbon nanotubes: a state of the art review," arXiv preprint arXiv:1112.4839, 2011.

[48] R. Ansari and M. Hemmatnezhad, "Nonlinear vibrations of embedded multi-walled carbon nanotubes using a variational approach," Mathematical and Computer Modelling, vol. 53, no. 5, pp. 927-938, 2011.

[49] M. Sadri, S. Mashrouteh, D. Younesian, and E. Esmailzadeh, "Nonlinear free vibration analysis of a double-walled carbon nanotube," in Nanotechnology (IEEE-NANO), 2014 IEEE 14th International Conference on, 2014, pp. 913-916.

[50] E. Cigeroglu and H. Samandari, "Nonlinear free Vibrations of curved double-walled carbon Nanotubes using differential quadrature method,” Physica E: Low-dimensional 
Systems and Nanostructures, vol. 64, pp. 95-105, 2014.

[51] F. de Borbón and D. Ambrosini, "On the influence of van der Waals coefficient on the transverse vibration of double-walled carbon nanotubes," Computational Materials Science, vol. 65, pp. 504-508, 2012.

[52] R. Ansari and M. Hemmatnezhad, "Nonlinear finite element analysis for vibrations of double-walled carbon nanotubes,” Nonlinear Dynamics, vol. 67, no. 1, pp. 373-383, 2012.

[53] F. Razafimahery and Baroudi, "Transverse vibration analysis of Euler-Bernoulli beam carrying point masse submerged in fluid media," International Journal of Engineering $\mid \&$ Technology, vol. 4, no. 2, pp. 369-380, 2015.

[54] G. Formica, W. Lacarbonara, and R. Alessi, "Vibrations of carbon nanotube-reinforced composites,” Journal of sound and vibration, vol. 329, no. 10, pp. 1875-1889, 2010.

[55] S. Adali, "Variational principles for multi-walled carbon nanotubes undergoing non-linear vibrations by semi-inverse method," Micro \& Nano Letters, vol. 4, no. 4, pp. 198-203, 2009.

[56] Y. Zhang, G. Liu, and X. Xie, "Free transverse vibrations of double-walled carbon nanotubes using a theory of nonlocal elasticity," Physical Review B, vol. 71, no. 19, p. 195404, 2005.

[57] A. Tylikowski, "Instability of Thermally Induced Vibrations of Carbon Nanotubes via Nonlocal Elasticity,” Journal of Thermal Stresses, vol. 35, no. 1-3, pp. 281-289, 2012.

[58] M. Hemmatnezhad and R. Ansari, "Finite element formulation for the free vibration analysis of embedded double-walled carbon nanotubes based on nonlocal Timoshenko beam theory,” Journal of Theoretical and Applied Physics, vol. 7, no. 1, pp. 1-10, 2013.

[59] A. Shakouri, R. Lin, and T. Ng, "Free flexural vibration studies of double-walled carbon nanotubes with different boundary conditions and modeled as nonlocal Euler beams via the Galerkin method,” Journal of Applied Physics, vol. 106, no. 9, p. 094307, 2009.

[60] Q. Wang, "Wave propagation in carbon nanotubes via nonlocal continuum mechanics," Journal of applied physics, vol. 98, p. 124301, 2005.

[61] J. Reddy, "Nonlocal theories for bending, buckling and vibration of beams," International Journal of Engineering Science, vol. 45, no. 2, pp. 288-307, 2007.

[62] P. Ponnusamy and A. Amuthalakshmi, "Vibration analysis of a viscous fluid conveying double-walled carbon nanotube," in Advanced Nanomaterials and Emerging Engineering Technologies (ICANMEET), 2013 International Conference on, 2013, pp. 231-236. 
[63] B. Wang and K. Wang, "Vibration analysis of embedded nanotubes using nonlocal continuum theory,” Composites Part B: Engineering, vol. 47, pp. 96-101, 2013.

[64] S. M. Hashemi, "Free Vibrational Analysis of Rotating Beam-like Structures: A Dynamic Finite Element Approach", Ph.D. Thesis, Department of Mechanical Engineering, Laval University, Quebec, Canada.

[65] S. Hashemi, M. Richard, and G. Dhatt, “A new Dynamic Finite Element (DFE) formulation for lateral free vibrations of Euler-Bernoulli spinning beams using trigonometric shape functions," Journal of Sound and Vibration, vol. 220, no. 4, pp. 601624, 1999.

[66] S. M. Hashemi and M. J. Richard, "A Dynamic Finite Element (DFE) method for free vibrations of bending-torsion coupled beams," Aerospace Science and Technology, vol. 4, no. 1, pp. 41-55, 2000.

[67] S. M. Hashemi and M. J. Richard, "Free vibrational analysis of axially loaded bendingtorsion coupled beams: a dynamic finite element," Computers I\& Structures, vol. 77, no. 6, pp. 711-724, 2000.

[68] M. Shavezipur and S. Hashemi, "Free vibration of triply coupled centrifugally stiffened nonuniform beams, using a refined dynamic finite element method," Aerospace Science and Technology, vol. 13, no. 1, pp. 59-70, 2009.

[69] S. M. Hashemi and E. J. Adique, "A quasi-exact dynamic finite element for free vibration analysis of sandwich beams,” Applied Composite Materials, vol. 17, no. 2, pp. 259-269, 2010.

[70] S. M. Hashemi and A. Roach, "Dynamic Finite Element Analysis of ExtensionalTorsional Coupled Vibration in Nonuniform Composite Beams," Applied Composite Materials, vol. 18, no. 6, pp. 521-538, 2011.

[71] N. H. Erdelyi and S. M. Hashemi, "A dynamic stiffness element for free vibration analysis of delaminated layered beams," Modelling and Simulation in Engineering, vol. 2012, p. 2, 2012.

[72] M. T. Towliat Kashani, S. Jayasinghe, and S. M. Hashemi, "On the Flexural-Torsional Vibration and Stability of Beams Subjected to Axial Load and End Moment,” Shock and Vibration, vol. 2014, 2014.

[73] M. T. Kashani, S. Jayasinghe, and S. M. Hashemi, "Dynamic Finite Element Analysis of Bending-Torsion Coupled Beams Subjected to Combined Axial Load and End Moment," Shock and Vibration, vol. 2015, 2015.

[74] J. Banerjee, "Dynamic Stiffness Matrix (DSM) for structural elements: a general approach,” Computers I\& structures, vol. 63, no. 1, pp. 101-103, 1997. 
[75] J. Banerjee and F. Williams, "Coupled bending-torsional dynamic stiffness matrix for Timoshenko beam elements,” Computers I\& Structures, vol. 42, no. 3, pp. 301-310, 1992.

[76] J. Banerjee and F. Williams, "Coupled bending-torsional dynamic stiffness matrix of an axially loaded Timoshenko beam element," International Journal of Solids and Structures, vol. 31, no. 6, pp. 749-762, 1994.

[77] J. Banerjee, "Development of an exact dynamic stiffness matrix for free vibration analysis of a twisted Timoshenko beam," Journal of Sound and Vibration, vol. 270, no. 1, pp. 379401, 2004.

[78] J. Bannerjee and F. Williams, "Exact dynamic stiffness matrix for composite Timoshenko beams with applications," Journal of sound and vibration, vol. 194, no. 4, pp. 573-585, 1996.

[79] J. Banerjee, "Free vibration of axially loaded composite Timoshenko beams using the dynamic stiffness matrix method," Computers I\& structures, vol. 69, no. 2, pp. 197-208, 1998.

[80] J. Banerjee, S. Guo, and W. Howson, "Exact dynamic stiffness matrix of a bendingtorsion coupled beam including warping," Computers I\& structures, vol. 59, no. 4, pp. 613-621, 1996.

[81] J. Banerjee, "Free vibration analysis of a twisted beam using the dynamic stiffness method," International Journal of Solids and Structures, vol. 38, no. 38, pp. 6703-6722, 2001.

[82] J. Banerjee, "Free vibration of sandwich beams using the dynamic stiffness method," Computers I\& structures, vol. 81, no. 18, pp. 1915-1922, 2003.

[83] J. Banerjee, H. Su, and D. Jackson, "Free vibration of rotating tapered beams using the dynamic stiffness method," Journal of Sound and Vibration, vol. 298, no. 4, pp. 10341054, 2006.

[84] L. Jun and H. Hongxing, "Dynamic stiffness vibration analysis of an elastically connected three-beam system,” Applied Acoustics, vol. 69, no. 7, pp. 591-600, 2008.

[85] L. Xiaobin, X. Shuangxi, W. Weiguo, and L. Jun, “An exact dynamic stiffness matrix for axially loaded double-beam systems,” Sadhana, vol. 39, no. 3, pp. 607-623, 2014.

[86] J. Banerjee, S. Papkov, X. Liu, and D. Kennedy, "Dynamic stiffness matrix of a rectangular plate for the general case," Journal of Sound and Vibration, vol. 342, pp. 177199, 2015.

[87] W. Wittrick and F. Williams, "A general algorithm for computing natural frequencies of elastic structures," The Quarterly Journal of Mechanics and Applied Mathematics, vol. 
24, no. 3, pp. 263-284, 1971.

[88] M. Paradise and T. Goswami, "Carbon nanotubes-production and industrial applications," Materials \& Design, vol. 28, no. 5, pp. 1477-1489, 2007.

[89] S. Iijima, "Helical microtubules of graphitic carbon," nature, vol. 354, no. 6348, pp. 5658, 1991.

[90] H. Boehm, "The first observation of carbon nanotubes," Carbon, vol. 35, no. 4, pp. 581584, 1997.

[91] M. Monthioux and V. L. Kuznetsov, "Who should be given the credit for the discovery of carbon nanotubes?,” Carbon, vol. 44, no. 9, pp. 1621-1623, 2006.

[92] L. Hofer, E. Sterling, and J. McCartney, "Structure of carbon deposited from carbon monoxide on iron, cobalt and nickel," The Journal of Physical Chemistry, vol. 59, no. 11, pp. 1153-1155, 1955.

[93] R. P. Feynman, "There's plenty of room at the bottom," Engineering and science, vol. 23, no. 5, pp. 22-36, 1960.

[94] R. Bacon, "Growth, structure, and properties of graphite whiskers," Journal of Applied Physics, vol. 31, no. 2, pp. 283-290, 1960.

[95] W. Bollmann and J. Spreadborough, "Action of graphite as a lubricant," Nature, vol. 186, pp. 29-30, 1960.

[96] M. L. Lieberman, C. R. Hills, and C. J. Miglionico, "Growth of graphite filaments," Carbon, vol. 9, no. 5, pp. 633-635, 1971.

[97] Tanuguchi. N, “On the basic concept of nano-technology,” 1974.

[98] Guston. G. H, Encyclopedia of Nanoscience and Society. Sage Publications, 2010.

[99] A. Oberlin, M. Endo, and T. Koyama, "Filamentous growth of carbon through benzene decomposition,” Journal of crystal growth, vol. 32, no. 3, pp. 335-349, 1976.

[100] Kroto. H. W. Heath. J. R. and S. O'Brien, “C60: Buckminsterfullerene,” Nature, vol. 318, no. 14, pp. 162-163, 1985.

[101] T. Ebbesen and P. Ajayan, "Large-scale synthesis of carbon nanotubes," Nature, vol. 358, no. 6383, pp. 220-222, 1992.

[102] S. Iijima and T. Ichihashi, "Single-shell carbon nanotubes of 1-nm diameter," 1993. 
[103] D. Bethune, C. Klang, M. De Vries, G. Gorman, R. Savoy, J. Vazquez, and R. Beyers, "Cobalt-catalysed growth of carbon nanotubes with single-atomic-layer walls," 1993.

[104] V. Sujatha, M. Suresh, and S. Mahalaxmi, “Carbon nanotubes (a review),” SRM University Journal of dental sciences, vol. 2, no. 4, 2011.

[105] D. Qian, G. J. Wagner, W. K. Liu, M. F. Yu, and R. S. Ruoff, "Mechanics of carbon nanotubes,” Applied Mechanics Reviews, vol. 55, p. 495, 2002.

[106] Y. Saito, T. Yoshikawa, S. Bandow, M. Tomita, and T. Hayashi, "Interlayer spacings in carbon nanotubes," Physical Review B, vol. 48, no. 3, p. 1907, 1993.

[107] R. S. Ruoff, D. Qian, and W. K. Liu, "Mechanical properties of carbon nanotubes: theoretical predictions and experimental measurements," Comptes Rendus Physique, vol. 4, no. 9, pp. 993-1008, 2003.

[108] C. Li and T.-W. Chou, "Elastic moduli of multi-walled carbon nanotubes and the effect of van der Waals forces," Composites Science and Technology, vol. 63, no. 11, pp. 15171524, 2003.

[109] G. Duesberg, W. Blau, H. J. Byrne, J. Muster, M. Burghard, and S. Roth, "Experimental observation of individual single-wall nanotube species by Raman microscopy," Chemical physics letters, vol. 310, no. 1, pp. 8-14, 1999.

[110] C. Journet, W. Maser, P. Bernier, A. Loiseau, M. L. de La Chapelle, de la S. Lefrant, P. Deniard, R. Lee, and J. Fischer, "Large-scale production of single-walled carbon nanotubes by the electric-arc technique," nature, vol. 388, no. 6644, pp. 756-758, 1997.

[111] T. Guo, P. Nikolaev, A. Thess, D. Colbert, and R. Smalley, "Catalytic growth of singlewalled manotubes by laser vaporization,” Chemical Physics Letters, vol. 243, no. 1, pp. 49-54, 1995.

[112] A. Guerrero, J. Puerta, F. Gomez, and F. Blanco, "Synthesis of carbon nanotubes by laser ablation in graphite substrate of industrial arc electrodes," Physica Scripta, vol. 2008, no. T131, p. 014007, 2008.

[113] A. Thess, R. Lee, P. Nikolaev, H. Dai, P. Petit, J. Robert, C. Xu, Y. H. Lee, S. G. Kim, A. G. Rinzler, and others, "Crystalline ropes of metallic carbon nanotubes," Science-AAASWeekly Paper Edition, vol. 273, no. 5274, pp. 483-487, 1996.

[114] E. Joselevich and C. M. Lieber, "Vectorial growth of metallic and semiconducting singlewall carbon nanotubes,” Nano Letters, vol. 2, no. 10, pp. 1137-1141, 2002.

[115] J. R. Arikatla, "VIBRATIONS OF SERIES OF BEAMS CONNECTED BY FLEXIBLE NONLINEAR LAYERS WITH APPLICATION TO CARBON NANOTUBES,” 2006. 
[116] X. Liu, Synthesis, Devices and Applications of Carbon Nanotubes. ProQuest, 2006.

[117] J. Xiao, "Study of factors affecting the synthesis of carbon nanotubes by spray pyrolysis," ProQuest, El Paso, 2007.

[118] Z. Yang, X. Chen, H. Nie, K. Zhang, W. Li, B. Yi, and L. Xu, "Direct synthesis of ultralong carbon nanotube bundles by spray pyrolysis and investigation of growth mechanism," Nanotechnology, vol. 19, no. 8, p. 085606, 2008.

[119] A. Dalila, A. B. Suriani, M. Rosmi, R. Rosazley, J. Rosli, and M. Rusop, “Carbon Nanotubes: A Brief Outlook on History, Synthesis Methods and Various BioHydrocarbon Sources,” in Advanced Materials Research, 2014, vol. 832, pp. 792-797.

[120] R. Kamalakaran, M. Terrones, T. Seeger, P. Kohler-Redlich, M. Rühle, Y. Kim, T. Hayashi, and M. Endo, "Synthesis of thick and crystalline nanotube arrays by spray pyrolysis,” Applied Physics Letters, vol. 77, no. 21, pp. 3385-3387, 2000.

[121] S. Black, “Carbon fiber market: Gathering momentum,” 2012. [Online]. Available: http://www.compositesworld.com/articles/carbon-fiber-market-gathering-momentum. [Accessed: 16-Dec-2015].

[122] “Realizing the Potential for Carbon Nanotubes,” 2014.

[123] P. Eklund, P. Ajayan, R. Blackmon, A. J. Hart, J. Kibng, B. Pradhan, A. Bao, and A. Rinzler, "International assessment of research and development of carbon nanotube manufacturing and applications," 2007.

[124] M. J. Treacy, T. Ebbesen, and J. Gibson, "Exceptionally high Young’s modulus observed for individual carbon nanotubes,” 1996.

[125] T. Kuzumaki, T. Hayashi, H. Ichinose, K. Miyazawa, K. Ito, and Y. Ishida, "In-situ observed deformation of carbon nanotubes,” Philosophical magazine A, vol. 77, no. 6, pp. 1461-1469, 1998.

[126] Z. Wang, P. Poncharal, and W. De Heer, "Measuring physical and mechanical properties of individual carbon nanotubes by in situ TEM," Journal of Physics and Chemistry of Solids, vol. 61, no. 7, pp. 1025-1030, 2000.

[127] E. W. Wong, P. E. Sheehan, and C. M. Lieber, "Nanobeam mechanics: elasticity, strength, and toughness of nanorods and nanotubes," Science, vol. 277, no. 5334, pp. 1971-1975, 1997.

[128] A. Krishnan, E. Dujardin, T. Ebbesen, P. Yianilos, and M. Treacy, "Young's modulus of single-walled nanotubes," Physical Review B, vol. 58, no. 20, p. 14013, 1998. 
[129] J.-P. Salvetat, G. A. D. Briggs, J.-M. Bonard, R. R. Bacsa, A. J. Kulik, T. Stöckli, N. A. Burnham, and L. Forró, "Elastic and shear moduli of single-walled carbon nanotube ropes,” Physical review letters, vol. 82, no. 5, p. 944, 1999.

[130] J.-P. Salvetat, A. J. Kulik, J.-M. Bonard, G. A. D. Briggs, T. Stöckli, K. Méténier, S. Bonnamy, F. Béguin, N. A. Burnham, and L. Forró, "Elastic modulus of ordered and disordered multiwall carbon nanotubes,” Advanced Materials, vol. 11, no. 2, pp. 161-165, 1999.

[131] P. Poncharal, Z. Wang, D. Ugarte, and W. A. De Heer, "Electrostatic deflections and electromechanical resonances of carbon nanotubes,” Science, vol. 283, no. 5407, pp. 1513-1516, 1999.

[132] M.-F. Yu, O. Lourie, M. J. Dyer, K. Moloni, T. F. Kelly, and R. S. Ruoff, "Strength and breaking mechanism of multiwall carbon nanotubes under tensile load,” Science, vol. 287, no. 5453, pp. 637-640, 2000.

[133] F. Li, H. Cheng, S. Bai, G. Su, and M. Dresselhaus, "Tensile strength of single-walled carbon nanotubes directly measured from their macroscopic ropes," Applied physics letters, vol. 77, no. 20, pp. 3161-3163, 2000.

[134] C. Wei, K. Cho, and D. Srivastava, "Tensile strength of carbon nanotubes under realistic temperature and strain rate,” Physical Review B, vol. 67, no. 11, p. 115407, 2003.

[135] M. B. Nardelli, B. I. Yakobson, and J. Bernholc, "Mechanism of strain release in carbon nanotubes,” Physical review B, vol. 57, no. 8, p. R4277, 1998.

[136] T. Belytschko, S. Xiao, G. Schatz, and R. Ruoff, "Atomistic simulations of nanotube fracture,” Physical Review B, vol. 65, no. 23, p. 235430, 2002.

[137] B. Yakobson, M. Campbell, C. Brabec, and J. Bernholc, "High strain rate fracture and Cchain unraveling in carbon nanotubes," Computational Materials Science, vol. 8, no. 4, pp. 341-348, 1997.

[138] M. Shokrieh and R. Rafiee, "A review of the mechanical properties of isolated carbon nanotubes and carbon nanotube composites," Mechanics of composite materials, vol. 46, no. 2, pp. 155-172, 2010.

[139] B. Chandra, J. Bhattacharjee, M. Purewal, Y.-W. Son, Y. Wu, M. Huang, H. Yan, T. F. Heinz, P. Kim, J. B. Neaton, and J. Hone, "Molecular-scale quantum dots from carbon nanotube heterojunctions.," Nano Lett., vol. 9, no. 4, pp. 1544-8, 2009.

[140] H. Dai, E. W. Wong, and C. M. Lieber, "Probing electrical transport in nanomaterials: conductivity of individual carbon nanotubes,” Science, vol. 272, no. 5261, pp. 523-526, 1996. 
[141] T. Ebbesen, H. Lezec, H. Hiura, J. Bennett, H. Ghaemi, and T. Thio, "Electrical conductivity of individual carbon nanotubes," 1996.

[142] S. J. Tans, M. H. Devoret, H. Dai, A. Thess, R. E. Smalley, L. Georliga, and C. Dekker, "Individual single-wall carbon nanotubes as quantum wires," Nature 386 (6624), 474477.(1997), 1997.

[143] P. Delaney, M. Di Ventra, and S. Pantelides, "Quantized conductance of multiwall carbon nanotubes,” Applied Physics Letters, vol. 75, no. 24, pp. 3787-3789, 1999.

[144] S. Li, Z. Yu, C. Rutherglen, and P. J. Burke, "Electrical properties of $0.4 \mathrm{~cm}$ long singlewalled carbon nanotubes,” Nano Letters, vol. 4, no. 10, pp. 2003-2007, 2004.

[145] J. Plombon, K. P. OBrien, F. Gstrein, V. M. Dubin, and Y. Jiao, "High-frequency electrical properties of individual and bundled carbon nanotubes," Applied physics letters, vol. 90, no. 6, pp. 063106-063106, 2007.

[146] S. I. Cha, K. T. Kim, K. H. Lee, C. B. Mo, Y. J. Jeong, and S. H. Hong, "Mechanical and electrical properties of cross-linked carbon nanotubes," Carbon, vol. 46, no. 3, pp. 482488, 2008.

[147] M. Nihei, A. Kawabata, D. Kondo, M. Horibe, S. Sato, and Y. Awano, "Electrical properties of carbon nanotube bundles for future via interconnects," Japanese journal of applied physics, vol. 44, no. 4R, p. 1626, 2005.

[148] Z. Ounaies, C. Park, K. Wise, E. Siochi, and J. Harrison, "Electrical properties of single wall carbon nanotube reinforced polyimide composites," Composites Science and Technology, vol. 63, no. 11, pp. 1637-1646, 2003.

[149] R. S. Ruoff and D. C. Lorents, "Mechanical and thermal properties of carbon nanotubes," carbon, vol. 33, no. 7, pp. 925-930, 1995.

[150] J. Che, T. Cagin, and W. A. Goddard III, “Thermal conductivity of carbon nanotubes,” Nanotechnology, vol. 11, no. 2, p. 65, 2000.

[151] J. Hone, M. Llaguno, M. Biercuk, A. Johnson, B. Batlogg, Z. Benes, and J. Fischer, "Thermal properties of carbon nanotubes and nanotube-based materials," Applied Physics A, vol. 74, no. 3, pp. 339-343, 2002.

[152] P. Kim, L. Shi, A. Majumdar, and P. L. McEuen, "Thermal transport measurements of individual multiwall nanotubes.,” Phys. Rev. Lett., vol. 87, no. 21, p. 215502, 2001.

[153] C. Yu, L. Shi, Z. Yao, D. Li, and A. Majumdar, "Thermal conductance and thermopower of an individual single-wall carbon nanotube," Nano letters, vol. 5, no. 9, pp. 1842-1846, 2005. 
[154] C.-W. Chang, D. Okawa, H. Garcia, A. Majumdar, and A. Zettl, “Breakdown of Fourier’s law in nanotube thermal conductors," Physical review letters, vol. 101, no. 7, p. 075903, 2008.

[155] E. Pop, D. Mann, Q. Wang, K. Goodson, and H. Dai, “Thermal conductance of an individual single-wall carbon nanotube above room temperature,” Nano letters, vol. 6, no. 1, pp. 96-100, 2006.

[156] M. F. L. De Volder, S. H. Tawfick, R. H. Baughman, and A. J. Hart, “Carbon nanotubes: present and future commercial applications.," Science, vol. 339, no. 6119, pp. 535-9, 2013.

[157] T. Hayashi and M. Endo, "Carbon nanotubes as structural material and their application in composites,” Composites Part B: Engineering, vol. 42, no. 8, pp. 2151-2157, 2011.

[158] M. Terrones, "Science and technology of the twenty-first century: synthesis, properties, and applications of carbon nanotubes," Annual review of materials research, vol. 33, no. 1, pp. 419-501, 2003.

[159] Q. Wang and B. Arash, "Nanoresonators in Sensors and Molecular Transportation: An Introduction to the Possibilities of Carbon Nanotubes and Graphene Sheets," Nanotechnology Magazine, IEEE, vol. 8, no. 4, pp. 29-37, 2014.

[160] K. Kiani, "Nanomechanical sensors based on elastically supported double-walled carbon nanotubes,” Applied Mathematics and Computation, vol. 270, pp. 216-241, 2015.

[161] K. Gong, Y. Yan, M. Zhang, L. SU, S. XIONG, and L. MAO, "Electrochemistry and electroanalytical applications of carbon nanotubes: a review,” Analytical sciences, vol. 21, no. 12, pp. 1383-1393, 2005.

[162] K. Balasubramanian and M. Burghard, "Biosensors based on carbon nanotubes.," Anal Bioanal Chem, vol. 385, no. 3, pp. 452-68, 2006.

[163] M. Trojanowicz, “Analytical applications of carbon nanotubes: a review,” TrAC trends in analytical chemistry, vol. 25, no. 5, pp. 480-489, 2006.

[164] S. Peretz and O. Regev, "Carbon nanotubes as nanocarriers in medicine,” Current Opinion in Colloid \& Interface Science, vol. 17, no. 6, pp. 360-368, 2012.

[165] Y. Lin, S. Taylor, H. Li, K. S. Fernando, L. Qu, W. Wang, L. Gu, B. Zhou, and Y.-P. Sun, "Advances toward bioapplications of carbon nanotubes," Journal of Materials Chemistry, vol. 14, no. 4, pp. 527-541, 2004.

[166] R. Hirlekar, M. Yamagar, H. Garse, M. Vij, and V. Kadam, "Carbon nanotubes and its applications: a review,” Asian Journal of Pharmaceutical and Clinical Research, vol. 2, 
no. 4, pp. 17-27, 2009.

[167] R. V. Mundra, X. Wu, J. Sauer, J. S. Dordick, and R. S. Kane, "Nanotubes in biological applications.,” Curr. Opin. Biotechnol., vol. 28, pp. 25-32, 2014.

[168] B. S. Harrison and A. Atala, "Carbon nanotube applications for tissue engineering," Biomaterials, vol. 28, no. 2, pp. 344-353, 2007.

[169] A. Fabbro, M. Prato, and L. Ballerini, "Carbon nanotubes in neuroregeneration and repair.,” Adv. Drug Deliv. Rev., vol. 65, no. 15, pp. 2034-44, 2013.

[170] N. Sinha and J. T. W. Yeow, “Carbon nanotubes for biomedical applications.," IEEE Trans Nanobioscience, vol. 4, no. 2, pp. 180-95, 2005.

[171] A. Bianco, K. Kostarelos, C. D. Partidos, and M. Prato, "Biomedical applications of functionalised carbon nanotubes,” Chemical Communications, no. 5, pp. 571-577, 2005.

[172] B. J. Landi, M. J. Ganter, C. D. Cress, R. A. DiLeo, and R. P. Raffaelle, “Carbon nanotubes for lithium ion batteries,” Energy I\& Environmental Science, vol. 2, no. 6, pp. 638-654, 2009.

[173] Y. N. Jurn, M. Malek, W.-W. Liu, H. K. Hoomod, and A. A. Kadhim, "Review-coating methods of carbon nanotubes and their potential applications," in Control System, Computing and Engineering (ICCSCE), 2014 IEEE International Conference on, 2014, pp. 118-123.

[174] R. H. Baughman, C. Cui, A. A. Zakhidov, Z. Iqbal, J. N. Barisci, G. M. Spinks, G. G. Wallace, A. Mazzoldi, D. De Rossi, A. G. Rinzler, and others, "Carbon nanotube actuators,” Science, vol. 284, no. 5418, pp. 1340-1344, 1999.

[175] B. Wei, R. Vajtai, and P. Ajayan, "Reliability and current carrying capacity of carbon nanotubes,” Applied Physics Letters, vol. 79, no. 8, pp. 1172-1174, 2001.

[176] F. Kreupl, A. P. Graham, G. Duesberg, W. Steinhögl, M. Liebau, E. Unger, and W. Hönlein, "Carbon nanotubes in interconnect applications," Microelectronic Engineering, vol. 64, no. 1, pp. 399-408, 2002.

[177] N. De Jonge and J.--M. Bonard, "Carbon nanotube electron sources and applications," Philosophical Transactions of the Royal Society of London A: Mathematical, Physical and Engineering Sciences, vol. 362, no. 1823, pp. 2239-2266, 2004.

[178] M. A. Meador, B. Files, J. Li, H. Manohara, D. Powell, and E. Powell, "Draft nanotechnology roadmap-technology area 10," National Aeronautics and Space Administration, Washington, DC, 2010. 
[179] F. Simonis and S. Schilthuizen, "Nanotechnology; Innovation Opportunities for Tomorrow’s Defence, Report TNO Science $\ \&$ Industry Future Technology Center, the Netherlands," 2006.

[180] R. F. Gibson, “A review of recent research on mechanics of multifunctional composite materials and structures,” Composite Structures, vol. 92, no. 12, pp. 2793-2810, 2010.

[181] S. E. O’Donnell and D. B. Smith, "Potential Impact of Carbon Nanotube Reinforced Polymer Composite on Commercial Heavy Aircraft,” 2005.

[182] S. E. O’Donnell, K. R. Sprong, and B. M. Haltli, "Potential impact of carbon nanotube reinforced polymer composite on commercial aircraft performance and economics,” in 4th AIAA Aviation Technology, Integration and Operations (ATIO) conference. Chicago, IL, 2004.

[183] W. Zhao, M. Li, Z. Zhang, and H. Peng, "Carbon nanotube based composites film heater for de-icing application,” in 14th European Conference on Composite Materials, 2010.

[184] D. Chung, "Damage detection using self-sensing concepts,” Proceedings of the Institution of Mechanical Engineers, Part G: Journal of Aerospace Engineering, vol. 221, no. 4, pp. 509-520, 2007.

[185] B. Laubscher, “Space Elevator Systems Level Analysis,” in 55 th International Astronautical Congress, 2004.

[186] S. S. Cohen and A. K. Misra, "Elastic oscillations of the space elevator ribbon,” Journal of Guidance, Control, and Dynamics, vol. 30, no. 6, pp. 1711-1717, 2007.

[187] N. M. Pugno, "On the strength of the carbon nanotube-based space elevator cable: from nanomechanics to megamechanics,” Journal of Physics: Condensed Matter, vol. 18, no. 33, p. S1971, 2006.

[188] N. Pugno, M. Schwarzbart, A. Steindl, and H. Troger, "On the stability of the track of the space elevator,” Acta Astronautica, vol. 64, no. 5, pp. 524-537, 2009.

[189] T. Kukowski, “Lightweight CAbles for Aerospace,” 2012.

[190] “Sustainable Alternative Jet Fuels,” 2013. [Online]. Available:

https:/www.faa.gov/about/office_org/headquarters_offices/apl/research/alternative_fuels/. [Accessed: 17-Dec-2015].

[191] M. Gagné and D. Therriault, “Lightning strike protection of composites,” Progress in Aerospace Sciences, vol. 64, pp. 1-16, 2014. 
[192] P. C. Kim and D. G. Lee, "Composite sandwich constructions for absorbing the electromagnetic waves,” Composite Structures, vol. 87, no. 2, pp. 161-167, 2009.

[193] S. Timoshenko, History of strength of materials: with a brief account of the history of theory of elasticity and theory of structures. Courier Corporation, 1953.

[194] X. Q. He, S. Kitipornchai, C. Wang, and K. Liew, "Modelling of van der Waals force for infinitesimal deformation of multi-walled carbon nanotubes treated as cylindrical shells," International journal of solids and structures, vol. 42, no. 23, pp. 6032-6047, 2005.

[195] K. Xu, X. Guo, and C. Ru, "Vibration of a double-walled carbon nanotube aroused by nonlinear intertube van der Waals forces," Journal of Applied Physics, vol. 99, no. 6, p. 064303, 2006.

[196] I. Elishakoff, Carbon Nanotubes and Nanosensors: Vibrations, Bukling, and Balistic Impact. John Wiley \& Sons, 2013.

[197] F. W. Williams and W. H. Wittrick, "Exact buckling and frequency calculations surveyed,” Journal of Structural Engineering, vol. 109, no. 1, pp. 169-187, 1983.

[198] D. Pentaras and I. Elishakoff, "Free vibration of triple-walled carbon nanotubes," Acta mechanica, vol. 221, no. 3-4, pp. 239-249, 2011.

[199] J. Phadikar and S. Pradhan, "Variational formulation and finite element analysis for nonlocal elastic nanobeams and nanoplates," Computational Materials Science, vol. 49, no. 3, pp. 492-499, 2010.

[200] H. Ehteshami and M. A. Hajabasi, "Analytical approaches for vibration analysis of multiwalled carbon nanotubes modeled as multiple nonlocal Euler beams," Physica E: Lowdimensional Systems and Nanostructures, vol. 44, no. 1, pp. 270-285, 2011. 


\section{Appendices}

A1 - Governing Equation for DWCNTs embedded in an elastic medium

$$
\begin{gathered}
E_{1} I_{1} w_{1}^{\prime \prime \prime}+\rho_{1} A_{1} \ddot{w}_{1}=c\left(w_{2}-w_{1}\right) \\
E_{2} I_{2} w_{2}^{\prime \prime \prime \prime}+\rho_{2} A_{2} \ddot{w}_{2}=-c\left(w_{2}-w_{1}\right)-k w_{2}
\end{gathered}
$$

Equation ( $A 1.1)$ remains essentially the same but Equation $(A 1.2)$ is modified as follows:

$$
E_{2} I_{2} w_{2}^{\prime \prime \prime \prime}+\rho_{2} A_{2} \ddot{w}_{2}=-w_{2}(c+k)-c w_{1}
$$

Further

$$
E_{2} I_{2} w_{2}^{\prime \prime \prime \prime}+\rho_{2} A_{2} \ddot{w}_{2}=-w_{2} \widehat{C}-c w_{1}
$$

with $\widehat{c}=c+k$. 


\section{A2 - Stiffness and Mass matrices of DWCNTs}

The elemental stiffness and mass matrices are symmetric $8 x 8$ matrices with each term given below. Mass matrix terms are represented by $m_{i j}$ and stiffness matrix terms use $k_{i j}$.

$$
\begin{aligned}
& m_{11}=\frac{156 A_{1} \rho_{1} L}{420} ; m_{12}=m_{21}=\frac{22 L^{2} A_{1} \rho_{1}}{420} ; m_{15}=m_{51}=\frac{54 A_{1} \rho_{1} L}{420} ; m_{16}=m_{61}=\frac{-13 L^{2} A_{1} \rho_{1}}{420} ; \\
& m_{22}=\frac{4 L^{3} A_{1} \rho_{1}}{420} ; m_{25}=m_{52}=\frac{13 L^{2} A_{1} \rho_{1}}{420} ; m_{26}=m_{62}=\frac{-3 L^{3} A_{1} \rho_{1}}{420} ; m_{33}=\frac{156 A_{2} \rho_{2} L}{420} ; \\
& m_{34}=m_{43}=\frac{22 L^{2} A_{2} \rho_{2}}{420} ; m_{37}=m_{73}=\frac{54 A_{2} \rho_{2} L}{420} ; m_{38}=m_{83}=\frac{-13 L^{2} A_{2} \rho_{2}}{420} ; m_{44}=\frac{4 L^{3} A_{2} \rho_{2}}{420} ; \\
& m_{47}=m_{74}=\frac{13 L^{2} A_{2} \rho_{2}}{420} ; m_{48}=m_{84}=\frac{-3 L^{3} A_{2} \rho_{2}}{420} ; m_{55}=\frac{156 A_{1} \rho_{1} L}{420} ; m_{56}=m_{65}=\frac{-22 L^{2} A_{1} \rho_{1}}{420} ; \\
& m_{66}=\frac{4 L^{3} A_{1} \rho_{1}}{420} ; m_{77}=\frac{156 A_{2} \rho_{2} L}{420} ; m_{78}=m_{87}=\frac{-22 L^{2} A_{2} \rho_{2}}{420} ; m_{88}=\frac{4 L^{3} A_{2} \rho_{2}}{420} ; \\
& m_{13}=m_{31}=m_{14}=m_{41}=m_{17}=m_{71}=m_{23}=m_{32}=m_{18}=m_{81}=m_{24}=m_{42}=m_{45}= \\
& m_{54}=m_{27}=m_{72}=m_{28}=m_{82}=m_{35}=m_{53}=m_{36}=m_{63}=m_{46}=m_{64}=m_{58}=m_{85}= \\
& m_{67}=m_{76}=m_{68}=m_{86}=0 \text {; } \\
& k_{11}=\frac{12 E_{1} I_{1}}{L^{3}}+\frac{156 L c}{420} ; k_{12}=k_{21}=\frac{6 L E_{1} I_{1}}{L^{3}}+\frac{22 L^{2} c}{420} ; k_{13}=k_{31}=-\frac{156 L c}{420} ; k_{14}=k_{41}=-\frac{22 L^{2} c}{420} ; \\
& k_{15}=k_{51}=-\frac{12 E_{1} I_{1}}{L^{3}}+\frac{54 L c}{420} ; k_{16}=k_{61}=\frac{6 L E_{1} I_{1}}{L^{3}}-\frac{13 L^{2} c}{420} ; k_{17}=k_{71}=-\frac{54 L c}{420} ; k_{18}=k_{81}=\frac{13 L^{2} c}{420} ; \\
& k_{22}=\frac{4 L^{2} E_{1} I_{1}}{L^{3}}+\frac{4 L^{3} c}{420} ; k_{23}=k_{32}=-\frac{22 L^{2} c}{420} ; k_{24}=k_{42}=-\frac{4 L^{3} c}{420} ; k_{25}=k_{52}=-\frac{6 L E_{1} I_{1}}{L^{3}}+\frac{13 L^{2} c}{420} ; \\
& k_{26}=k_{62}=\frac{2 L^{2} E_{1} I_{1}}{L^{3}}-\frac{3 L^{3} c}{420} ; k_{27}=k_{72}=-\frac{13 L^{2} c}{420} ; k_{28}=k_{82}=\frac{3 L^{3} c}{420} ; \\
& k_{33}=\frac{12 E_{2} I_{2}}{L^{3}}+\frac{156 L \hat{c}}{420} ; k_{34}=k_{43}=\frac{6 L E_{2} I_{2}}{L^{3}}+\frac{22 L^{2} \hat{c}}{420} ; k_{35}=k_{53}=-\frac{54 L c}{420} ; k_{36}=k_{63}=\frac{13 L^{2} c}{420} ; \\
& k_{37}=k_{73}=-\frac{12 E_{2} I_{2}}{L^{3}}+\frac{54 L \hat{c}}{420} ; k_{38}=k_{83}=\frac{6 L E_{2} I_{2}}{L^{3}}-\frac{13 L^{2} \hat{c}}{420} ; \\
& k_{44}=\frac{4 L^{2} E_{1} I_{1}}{L^{3}}+\frac{4 L^{3} c}{420} ; k_{45}=k_{54}=-\frac{13 L^{2} c}{420} ; k_{46}=k_{64}=\frac{3 L^{3} c}{420} ; k_{47}=k_{74}=-\frac{6 L E_{2} I_{2}}{L^{3}}+\frac{13 L^{2} \hat{c}}{420} ; \\
& k_{48}=k_{84}=\frac{2 L^{2} E_{2} I_{2}}{L^{3}}-\frac{3 L^{3} \hat{c}}{420}
\end{aligned}
$$




$$
\begin{aligned}
& k_{55}=\frac{12 E_{1} I_{1}}{L^{3}}+\frac{156 L c}{420} ; k_{56}=k_{65}=-\frac{6 L E_{2} I_{2}}{L^{3}}-\frac{22 L^{2} \hat{c}}{420} ; k_{57}=k_{75}=-\frac{156 L c}{420} ; k_{58}=k_{85}=\frac{22 L^{2} c}{420} ; \\
& k_{66}=\frac{4 L^{2} E_{1} I_{1}}{L^{3}}+\frac{4 L^{3} c}{420} ; k_{67}=k_{76}=\frac{22 L^{2} c}{420} ; k_{68}=k_{86}=-\frac{4 L^{3} c}{420} ; \\
& k_{77}=\frac{12 E_{2} I_{2}}{L^{3}}+\frac{156 L \hat{c}}{420} ; k_{78}=k_{87}=-\frac{6 L E_{2} I_{2}}{L^{3}}-\frac{22 L^{2} \hat{c}}{420} ; k_{88}=\frac{4 L^{2} E_{1} I_{1}}{L^{3}}+\frac{4 L^{3} c}{420} ;
\end{aligned}
$$




\section{A3 - Mode shapes of DWCNTs embedded in elastic matrix}

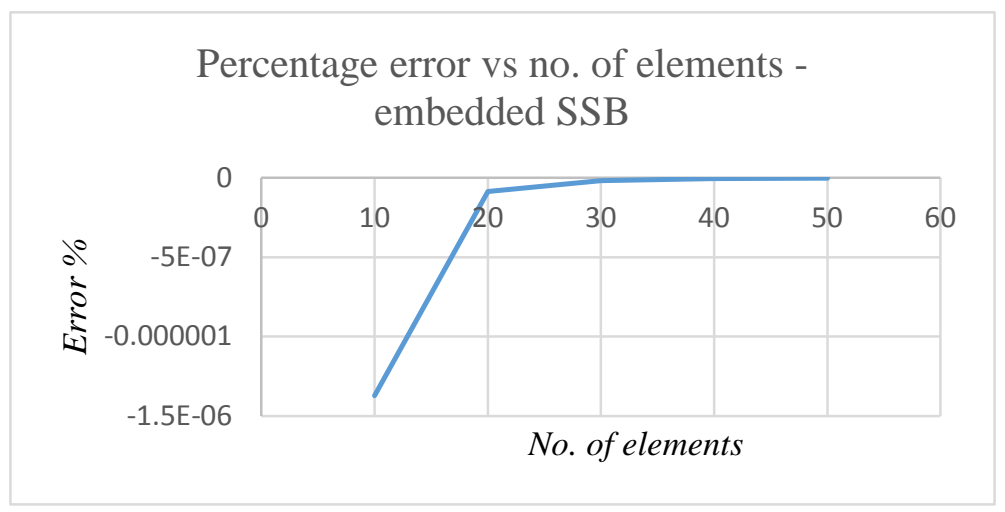

Figure A3.1 - Percentage error vs number of elements - embedded SSB - 50 FEM elements -

$$
(k / c=100)
$$

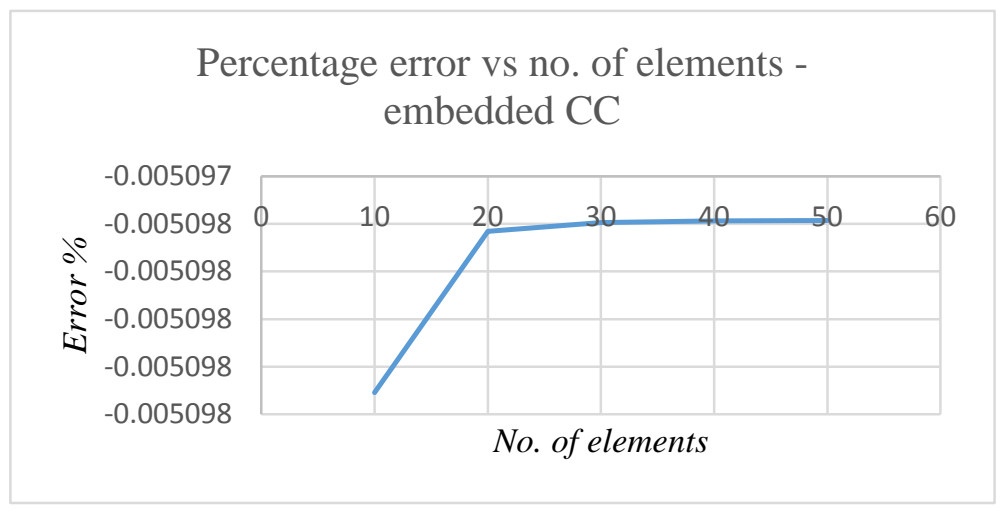

Figure A3.2 - Percentage error vs number of elements - embedded CC - 50 FEM elements -

$$
(k / c=100)
$$

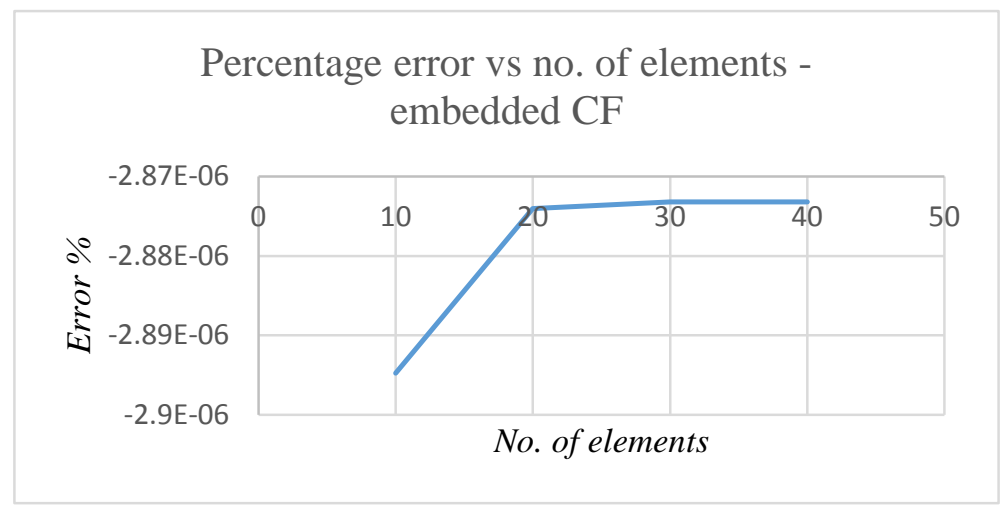

Figure A3.3 - Percentage error vs number of elements - embedded CF - 40 FEM elements -

$$
(k / c=100)
$$




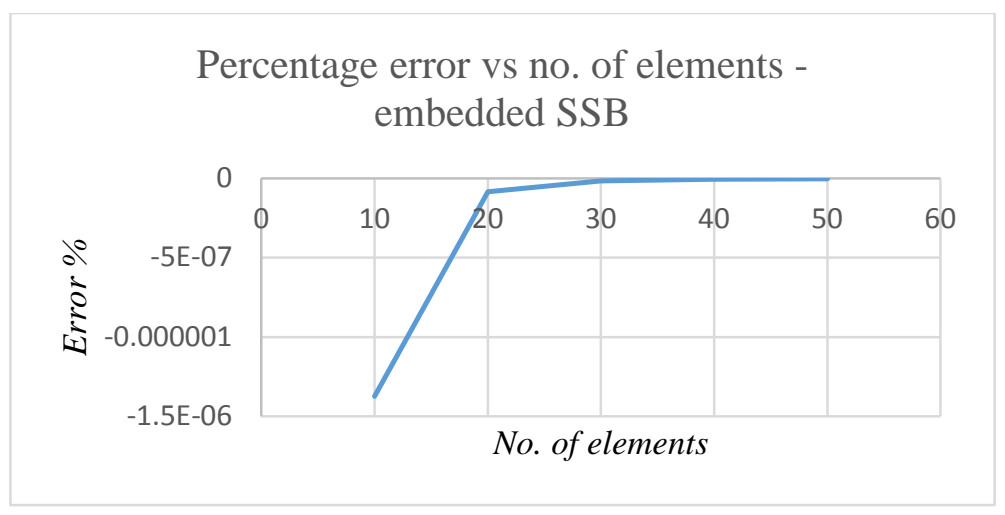

Figure A3.4 - Percentage error vs number of elements - embedded SSB - 50 FEM elements -

$$
(k / c=0.1)
$$

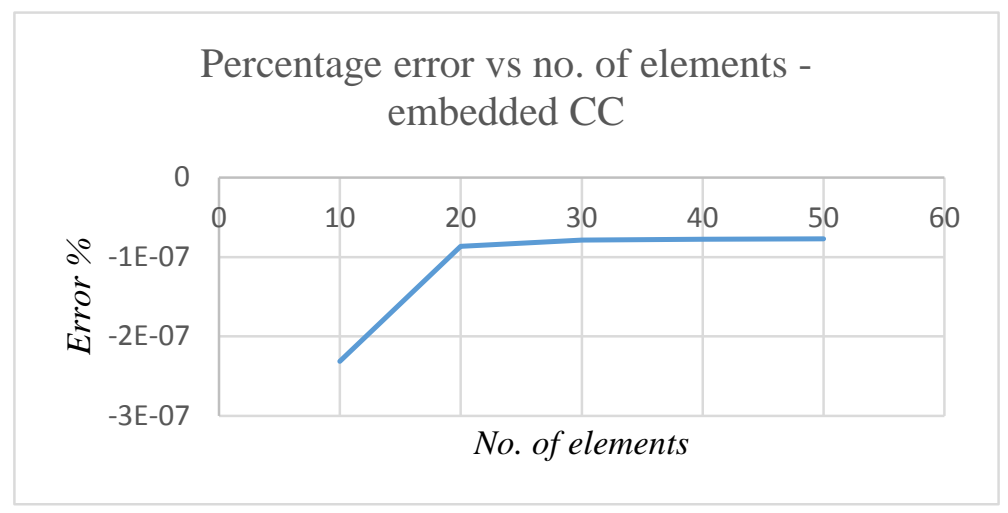

Figure A3.5 - Percentage error vs number of elements - embedded CC - 50 FEM elements -

$$
(k / c=0.1)
$$

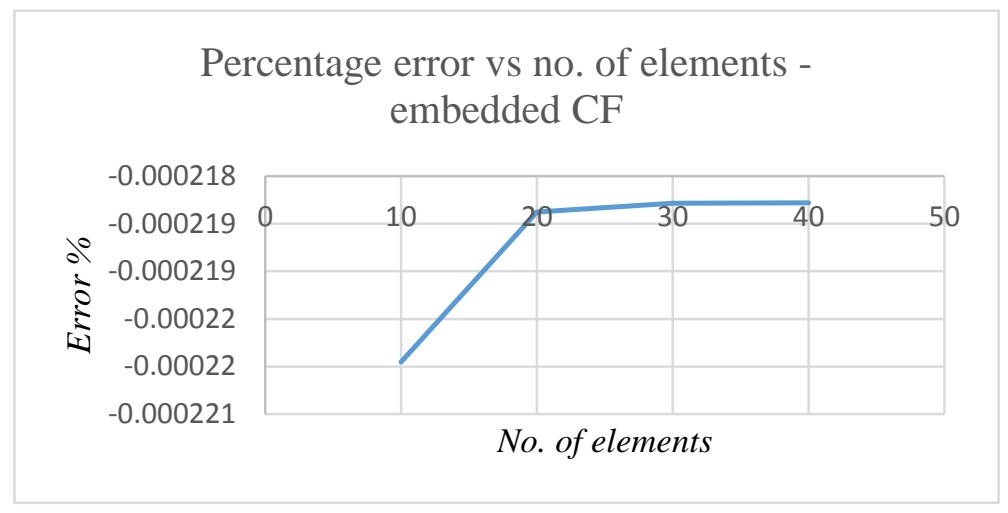

Figure A3. 6 - Percentage error vs number of elements - embedded CF - 40 FEM elements -

$$
(k / c=0.1)
$$




\section{A4 - Expression for clamped clamped natural frequency of DWCNTs}

The determinant of the $K$ matrix obtained in (4.36) is found to be

$$
\bar{D}=-4 \sqrt{\alpha} \sqrt{\beta}\left(4 \cos \left(\alpha^{1 / 4}\right) \cosh \left(\alpha^{1 / 4}\right)+4 \cos \left(\beta^{1 / 4}\right) \cosh \left(\beta^{1 / 4}\right)-4 \cos \left(\alpha^{1 / 4}\right) \cosh \left(\alpha^{1 / 4}\right) \cos \left(\beta^{1 / 4}\right) \cosh \left(\beta^{1 / 4}\right)-4\right)
$$

For the roots to be found, determinant of the matrix has to go to zero. (A4.1) then is equated to zero and either

$\cos \left(\alpha^{1 / 4}\right) \cosh \left(\alpha^{1 / 4}\right)-1=0$

or

$\cos \left(\beta^{1 / 4}\right) \cosh \left(\beta^{1 / 4}\right)-1=0$

$\alpha=0$ or $\beta=0$ satisfies (A4.2) and (A4.3)

Substituting $\alpha, \beta$ in (4.12) and (4.13) and using (4.8) and (4.9)/(4.9.1) gives

$\omega^{2}=\frac{c\left(m_{1}+m_{2}\right)}{m_{1} m_{2}}$

which is the natural frequency for clamped clamped case of DWCNTs. 
A5 - Governing equations for TWCNT embedded in an elastic matrix

$$
\begin{gathered}
E_{1} I_{1} w_{1}^{\prime \prime \prime \prime}+\rho_{1} A_{1} \ddot{w}_{1}=c_{1} w_{2}-c_{1} w_{1} \\
E_{2} I_{2} w_{2}^{\prime \prime \prime \prime}+\rho_{2} A_{2} \ddot{w}_{2}=c_{2} w_{3}-c_{2} w_{2}-c_{1} w_{2}+c_{1} w_{1} \\
E_{3} I_{3} w_{3}^{\prime \prime \prime \prime}+\rho_{3} A_{3} \ddot{w}_{3}=-\widehat{c}_{2} w_{3}+c_{2} w_{2}
\end{gathered}
$$

For an embedded case, equation ( $A 5.3)$ changes as

$$
E_{3} I_{3} w_{3}^{\prime \prime \prime}+\rho_{3} A_{3} \ddot{w}_{3}=-\left(c_{2}+k\right) k w_{3}+c_{2} w_{2}
$$

and

$$
E_{3} I_{3} w_{3}^{\prime \prime \prime}+\rho_{3} A_{3} \ddot{w}_{3}=-\widehat{c}_{2} w_{3}+c_{2} w_{2}
$$

with $\hat{c}_{2}=c_{2}+k$ 


\section{A6 - Stiffness and mass matrices of TWCNTs}

The form of the elemental mass and stiffness matrix is of the form given below. Further the mass and stiffness elements are defined as $m_{i j}$ and $k_{i j}$ respectively.

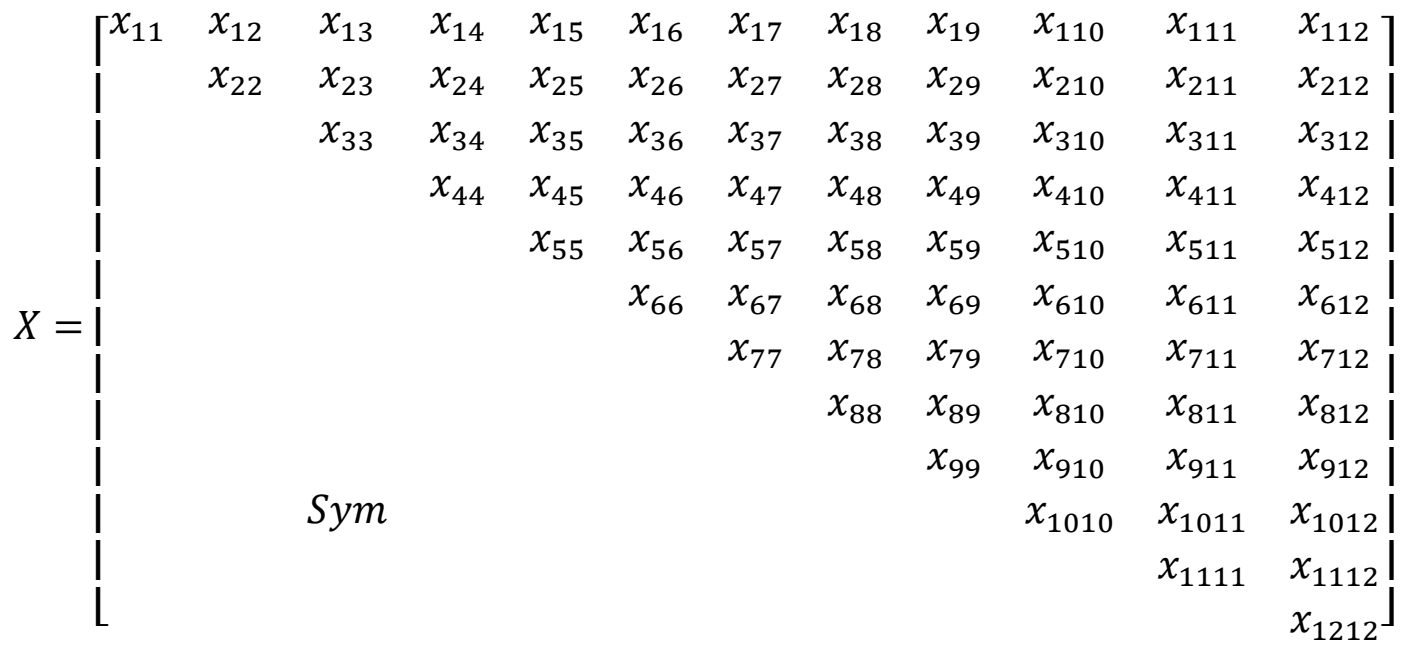

$$
\begin{aligned}
& m_{11}=\frac{156 m_{1} l}{420} ; m_{12}=m_{21}=\frac{22 l^{2} m_{1}}{420} ; m_{17}=m_{71}=\frac{54 m_{1} l}{420} ; m_{18}=m_{81}=\frac{-13 l^{2} m_{1}}{420} ; \\
& m_{22}=\frac{4 l^{3} m_{1}}{420} ; m_{27}=m_{72}=\frac{13 l^{2} m_{1}}{420} ; m_{28}=m_{82}=\frac{-3 l^{3} m_{1}}{420} ; \\
& m_{33}=\frac{156 l m_{2}}{420} ; m_{34}=m_{43}=\frac{22 l^{2} m_{1}}{420} ; m_{39}=m_{93}=\frac{54 m_{2} l}{420} ; m_{310}=m_{103}=\frac{-13 l^{2} m_{2}}{420} ; \\
& m_{44}=\frac{4 l^{3} m_{2}}{420} ; m_{49}=m_{94}=\frac{13 l^{2} m_{2}}{420} ; m_{410}=m_{104}=\frac{-3 l^{3} m_{2}}{420} ; \\
& m_{55}=\frac{156 m_{3} l}{420} ; m_{56}=m_{65}=\frac{22 l^{2} m_{3}}{420} ; m_{511}=m_{1105}=\frac{54 m_{3} l}{420} ; m_{512}=m_{1205}=\frac{-13 l^{2} m_{3}}{420} ; \\
& m_{66}=\frac{4 l^{3} m_{2}}{420} ; m_{611}=m_{1106}=\frac{13 l^{2} m_{2}}{420} ; m_{612}=m_{1206}=\frac{-3 l^{3} m_{3}}{420} ; m_{77}=\frac{156 m_{1} l}{420} ; \\
& m_{78}=m_{87}=\frac{-22 m_{1} l^{2}}{420} ; m_{88}=\frac{4 l^{3} m_{1}}{420} ; m_{99}=\frac{156 l m_{2}}{420} ; m_{910}=m_{109}=\frac{-22 m_{2} l^{2}}{420} ; \\
& m_{1010}=\frac{4 l^{3} m_{2}}{420} ; m_{1111}=\frac{156 m_{3} l}{420} ; m_{1112}=m_{1211}=\frac{-22 m_{3} l^{2}}{420} ; m_{1212}=\frac{4 l^{3} m_{3}}{420} ; \\
& k_{11}=\frac{12 E_{1} I_{1}}{l^{3}}+\frac{156 l c_{1}}{420} ; k_{12}=k_{21}=\frac{6 l E_{1} I_{1}}{l^{3}}+\frac{22 l^{2} c_{1}}{420} ; k_{13}=k_{31}=\frac{-156 l c_{1}}{420} ; k_{14}=k_{41}=\frac{-22 l^{2} c_{1}}{420} ; \\
& k_{17}=k_{71}=\frac{-12 E_{1} I_{1}}{l^{3}}+\frac{54 l c_{1}}{420} ; k_{18}=k_{81}=\frac{6 l E_{1} I_{1}}{l^{3}}-\frac{13 l^{3} c_{1}}{420} k_{19}=k_{91}=\frac{-54 l c_{1}}{420} \text {; }
\end{aligned}
$$




$$
\begin{aligned}
& k_{110}=k_{101}=\frac{13 l^{2} c_{1}}{420} ; k_{22}=\frac{4 l^{2} E_{1} I_{1}}{l^{3}}+\frac{4 l^{3} c_{1}}{420} ; k_{23}=k_{32}=\frac{-22 l^{2} c_{1}}{420} ; k_{24}=k_{42}=\frac{-4 l^{3} c_{1}}{420} ; \\
& k_{27}=k_{72}=\frac{-6 l E_{1} I_{1}}{l^{3}}+\frac{13 l^{3} c_{1}}{420} ; k_{28}=k_{82}=\frac{2 l^{2} E_{1} I_{1}}{l^{3}}-\frac{3 l^{3} c_{1}}{420} ; k_{29}=k_{92}=\frac{-13 l^{2} c_{1}}{420} ; \\
& k_{210}=k_{102}=\frac{3 l^{3} c_{1}}{420} ; k_{33}=\frac{12 E_{1} I_{1}}{l^{3}}+\frac{156 l\left(c_{1}+c_{2}\right)}{420} ; k_{34}=k_{43}=\frac{6 l E_{1} I_{1}}{l^{3}}+\frac{22 l^{2}\left(c_{1}+c_{2}\right)}{420} ; \\
& k_{35}=k_{53}=\frac{-156 l c_{2}}{420} ; k_{36}=k_{63}=\frac{-22 l^{2} c_{2}}{420} ; k_{37}=k_{73}=\frac{-54 l c_{1}}{420} ; k_{38}=k_{83}=\frac{13 l^{2} c_{1}}{420} ; \\
& k_{39}=k_{93}=\frac{-12 E_{2} I_{2}}{l^{3}}+\frac{54 l\left(c_{1}+c_{2}\right)}{420} ; k_{310}=k_{103}=\frac{6 l E_{1} I_{1}}{l^{3}}-\frac{13 l^{2}\left(c_{1}+c_{2}\right)}{420} ; k_{311}=k_{1103}=\frac{-54 c_{2} l}{420} ; \\
& k_{312}=k_{1203}=\frac{13 l^{2} c_{2}}{420} ; k_{44}=\frac{4 l^{3} E_{2} I_{2}}{l^{3}}+\frac{4 l^{3}\left(c_{1}+c_{2}\right)}{420} ; k_{45}=k_{54}=\frac{-22 l^{2} c_{2}}{420} ; k_{46}=k_{64}=\frac{-4 l^{3} c_{2}}{420} ; \\
& k_{47}=k_{74}=\frac{-13 l^{2} c_{1}}{420} ; k_{48}=k_{84}=\frac{3 l^{3} c_{1}}{420} ; k_{49}=k_{94}=\frac{-6 l E_{2} I_{2}}{l^{3}}+\frac{13 l^{2}\left(c_{1}+c_{2}\right)}{420} ; \\
& k_{410}=k_{104}=\frac{2 l^{2} E_{2} I_{2}}{l^{3}}-\frac{3 l^{3}\left(c_{1}+c_{2}\right)}{420} ; k_{411}=k_{1104}=\frac{-13 l^{2} c_{2}}{420} ; k_{412}=k_{1204}=\frac{3 l^{3} c_{2}}{420} ; \\
& k_{55}=\frac{12 E_{3} I_{3}}{l^{3}}+\frac{156 l c_{2}}{420} ; k_{56}=k_{65}=\frac{6 l E_{3} I_{3}}{l^{3}}+\frac{22 l^{2} c_{2}}{420} ; k_{59}=k_{95}=\frac{-54 c_{2} l}{420} ; k_{510}=k_{105}=\frac{13 l^{2} c_{2}}{420} ; \\
& k_{511}=k_{1105}=\frac{-12 E_{3} I_{3}}{l^{3}}+\frac{54 l c_{2}}{420} ; k_{512}=k_{1205}=\frac{6 l E_{3} I_{3}}{l^{3}}-\frac{13 l^{2} c_{2}}{420} ; k_{66}=\frac{4 l^{2} E_{3} I_{3}}{l^{3}}+\frac{4 l^{3} c_{2}}{420} ; \\
& k_{69}=k_{96}=\frac{-13 l^{2} c_{2}}{420} ; k_{610}=k_{106}=\frac{3 l^{3} c_{2}}{420} ; k_{611}=k_{1106}=\frac{-6 l E_{3} I_{3}}{l^{3}}+\frac{13 l^{3} c_{2}}{420} ; \\
& k_{612}=k_{1206}=\frac{2 l^{2} E_{3} I_{3}}{l^{3}}-\frac{3 l^{3} c_{2}}{420} k_{77}=\frac{12 E_{1} I_{1}}{l^{3}}+\frac{156 l c_{1}}{420} ; k_{78}=k_{87}=-\frac{6 l E_{1} I_{1}}{l^{3}}-\frac{22 l^{2} c_{1}}{420} ; \\
& k_{79}=k_{97}=\frac{-156 l c_{1}}{420} ; k_{710}=k_{107}=\frac{22 l^{2} c_{1}}{420} ; k_{88}=\frac{4 l^{2} E_{1} I_{1}}{l^{3}}+\frac{4 l^{3} c_{1}}{420} ; k_{89}=k_{98}=\frac{22 l^{2} c_{1}}{420} ; \\
& k_{810}=k_{108}=\frac{-4 l^{3} c_{1}}{420} ; k_{99}=\frac{12 E_{2} I_{2}}{l^{3}}+\frac{156 l\left(c_{1}+c_{2}\right)}{420} ; k_{910}=k_{109}=-\frac{6 l E_{2} I_{2}}{l^{3}}-\frac{22 l^{2}\left(c_{1}+c_{2}\right)}{420} ; \\
& k_{911}=k_{1109}=\frac{-156 c_{2} l}{420} ; k_{912}=k_{1209}=\frac{22 l^{2} c_{2}}{420} ; k_{1010}=\frac{4 l^{3} E_{2} I_{2}}{l^{3}}+\frac{4 l^{3}\left(c_{1}+c_{2}\right)}{420} ; \\
& k_{1011}=k_{1110}=\frac{22 l^{2} c_{2}}{420} ; k_{1012}=k_{1012}=\frac{-4 l^{3} c_{2}}{420} ; k_{1111}=\frac{12 E_{3} I_{3}}{l^{3}}+\frac{156 l c_{2}}{420} ; \\
& k_{1112}=k_{1211}=-\frac{6 l E_{3} I_{3}}{l^{3}}-\frac{22 l^{2} c_{2}}{420} k_{1212}=\frac{4 l^{2} E_{3} I_{3}}{l^{3}}+\frac{4 l^{3} c_{2}}{420} ;
\end{aligned}
$$


A7 - Mode shapes of TWCNTs embedded in elastic matrix

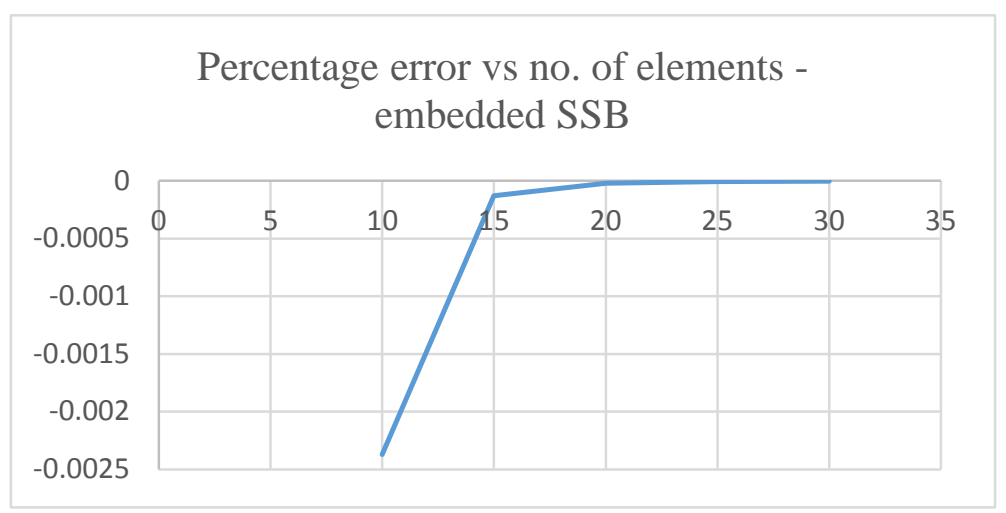

Figure A7.1 - Percentage error vs number of elements - embedded SSB - 30 FEM elements -

$$
(k / c=0.1)
$$

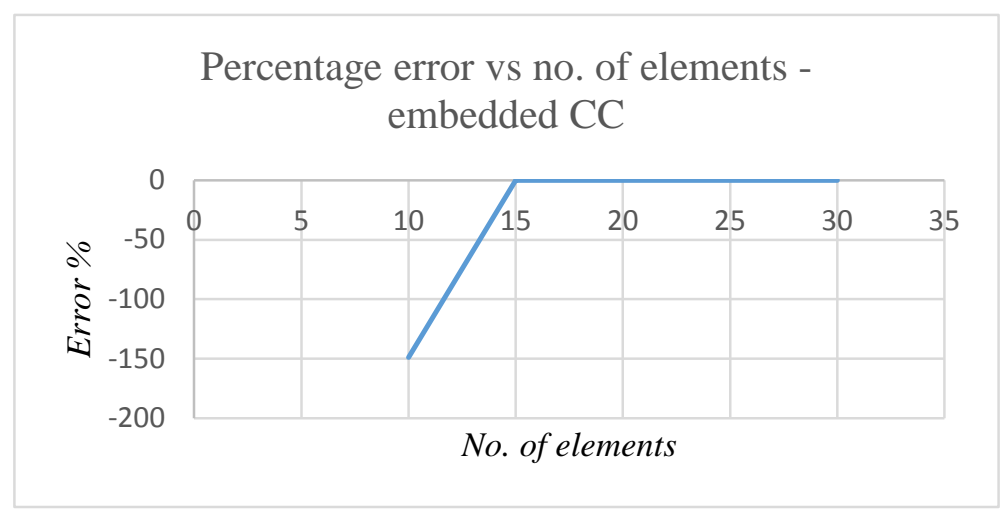

Figure A7.2 - Percentage error vs number of elements - embedded CC - 30 FEM elements -

$$
(k / c=0.1)
$$

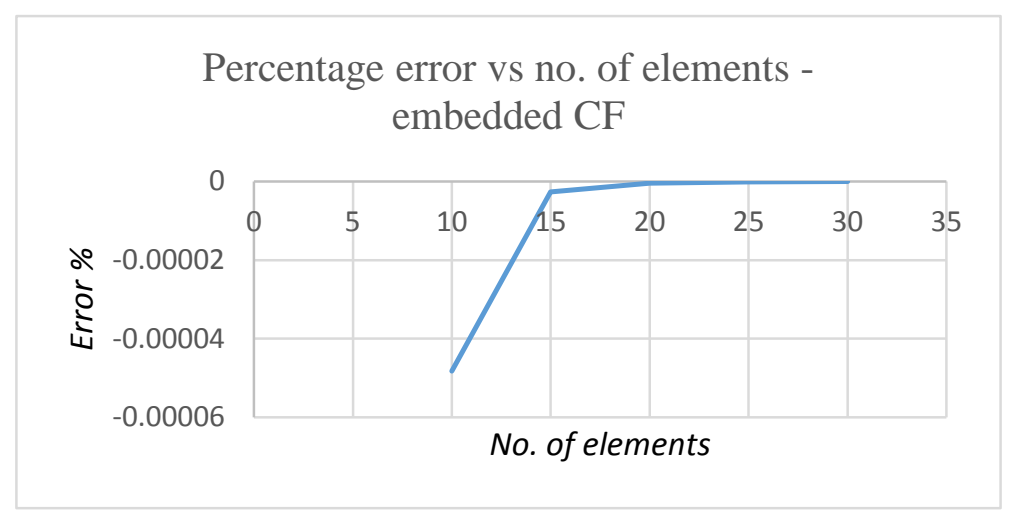

Figure A7.3 - Percentage error vs number of elements - embedded CF - 30 FEM elements -

$$
(k / c=0.1)
$$




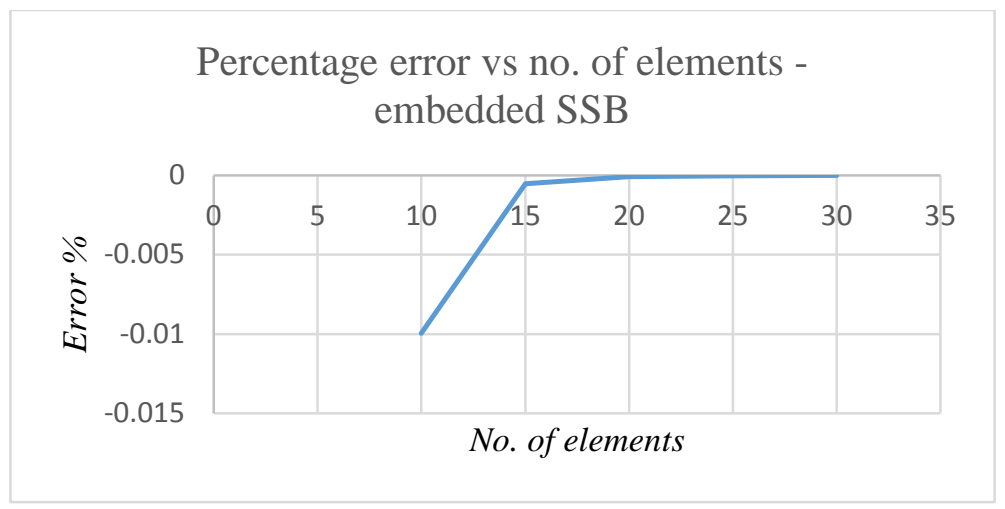

Figure A7.4 - Percentage error vs number of elements - embedded SSB - 30 FEM elements $(k / c=0.0001)$

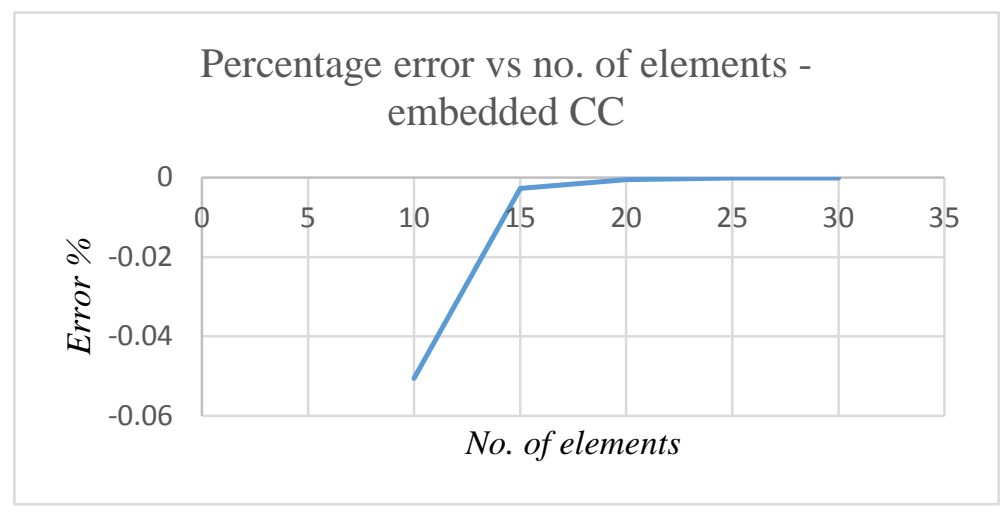

Figure A7-5 - Percentage error vs number of elements - embedded CC - 30 FEM elements -

$$
(k / c=0.0001)
$$

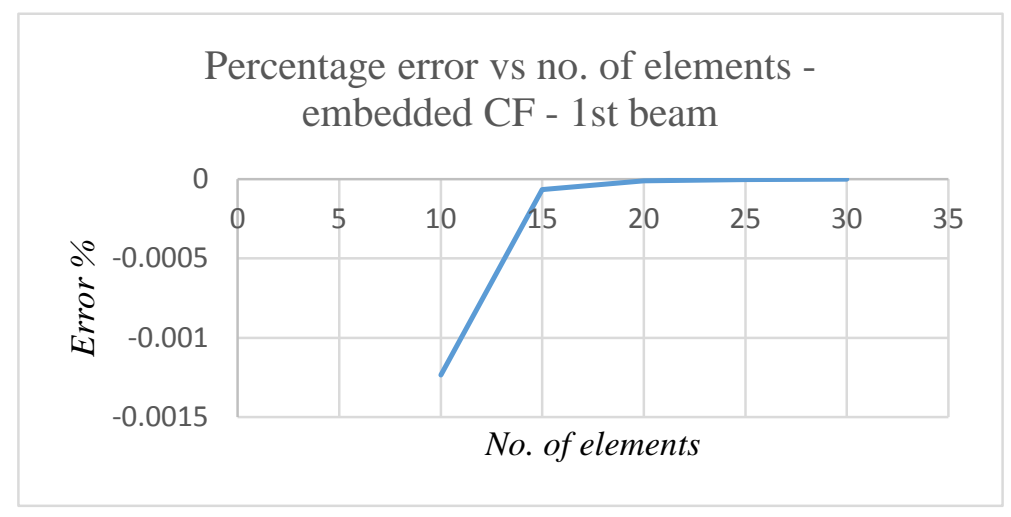

Figure A7.6 - Percentage error vs number of elements - embedded CF - 30 FEM elements -

$$
(k / c=0.0001)
$$




\section{A8 - Dynamic Stiffness Matrix (DSM) for TWCNTs}

The governing equations for a triple-walled carbon nanotube, assuming simple harmonic motion is given as

$$
\begin{gathered}
E_{1} I_{1} W_{1}^{\prime \prime \prime \prime}+\left(c_{1}-\rho_{1} A_{1} \omega^{2}\right) W_{1}-c_{1} W_{2}=0 \\
E_{2} I_{2} W^{\prime \prime \prime \prime}+\left(c_{1}+c_{2}-\rho_{2} A_{2} \omega^{2}\right) W_{2}-c_{2} W_{3}-c_{1} W_{2}=0 \\
E_{3} I_{3} W^{\prime \prime \prime \prime}+\left(\hat{c}_{2}-\rho_{3} A_{3} \omega^{2}\right) W_{3}-c_{2} W_{2}=0
\end{gathered}
$$

Assuming $D=d / d x$ and rewriting $(A 8.1)-(A 8.3)$ as

$$
\begin{gathered}
\left(E_{1} I_{1} D^{4}+\left(c_{1}-\rho_{1} A_{1} \omega^{2}\right)\right) W_{1}-c_{1} W_{2}=0 \\
\left(E_{2} I_{2} D^{4}+\left(c_{1}+c_{2}-\rho_{2} A_{2} \omega^{2}\right)\right) W_{2}-c_{2} W_{3}-c_{1} W_{2}=0 \\
\left(E_{3} I_{3} D^{4}+\left(c_{2}-\rho_{3} A_{3} \omega^{2}\right)\right) W_{3}-c_{2} W_{2}=0
\end{gathered}
$$

Combining (A8.4) - (A8.6) into a single equation as

$$
\left(D^{12}+p D^{8}+q\right) Y=0
$$

The equation can be reduced to a cubic equation by taking $D^{4}=\kappa$

$$
\left(\kappa^{3}+p \kappa^{2}+q\right) Y=0
$$

The roots of the cubic equation can be found by following the usual procedure. It is to be noted that the roots are to be of the form [real \& imaginary], [real \& complex] and [complex]. Any combination involving [real, imaginary \& complex] can’t deduce the dynamic stiffness matrix, to the best of author's knowledge. These roots seem to be elusive at the moment and would form the future research priorities for the author. 


\section{A9 - Dynamic Stiffness Matrix (DSM) for nonlocal DWCNTs}

The governing equations of nonlocal DWCNTs are given by

$$
\begin{gathered}
E_{1} I_{1} \frac{\partial^{4} w_{1}}{\partial x^{4}}+\rho_{1} A_{1} \frac{\partial^{2} w_{1}}{\partial t^{2}}-\mu^{2} \rho_{1} A_{1} \frac{\partial^{4} w_{1}}{\partial x^{2} \partial t^{2}}-c\left(w_{2}-w_{1}\right)+\mu^{2} c\left(\frac{\partial^{2} w_{2}}{\partial x^{2}}-\frac{\partial^{2} w_{1}}{\partial x^{2}}\right)=0 \\
E_{2} I_{2} \frac{\partial^{4} w_{2}}{\partial x^{4}}+\rho_{2} A_{2} \frac{\partial^{2} w_{2}}{\partial t^{2}}-\mu^{2} \rho_{2} A_{2} \frac{\partial^{4} w_{2}}{\partial x^{2} \partial t^{2}}-c\left(w_{1}-w_{2}\right)+\mu^{2} c\left(\frac{\partial^{2} w_{1}}{\partial x^{2}}-\frac{\partial^{2} w_{2}}{\partial x^{2}}\right)=0
\end{gathered}
$$

By removing time dependency and assuming simple harmonic motion

$$
\begin{aligned}
& E_{1} I_{1} W_{1}^{\prime \prime \prime \prime}-m_{1} \omega^{2} W_{1}+\mu^{2} m_{1} W_{1}^{\prime \prime}-c\left(W_{2}-W_{1}\right)+\mu^{2} c\left(W_{2}^{\prime \prime}-W_{1}^{\prime \prime}\right)=0 \\
& E_{2} I_{2} W_{2}^{\prime \prime \prime \prime}-m_{2} \omega^{2} W_{2}+\mu^{2} m_{2} W_{2}^{\prime \prime}-c\left(W_{2}-W_{1}\right)+\mu^{2} c\left(W_{1}^{\prime \prime}-W_{2}^{\prime \prime}\right)=0
\end{aligned}
$$

Equations ( $A 9.2)$ can be combined as a single equation as

$$
D^{8}+a_{1} D^{6}+a_{2} D^{4}+a_{3} D^{2}+a_{4}=0
$$

with

$$
\begin{aligned}
& a_{1}=\frac{E_{2} I_{2} m_{1} \mu^{2} \omega^{2}-E_{1} I_{1} c \mu^{2}-E_{2} I_{2} c \mu^{2}}{E_{1} I_{1} E_{2} I_{2}} \\
& a_{2}=\frac{-c m_{1} \mu^{4} \omega^{2}+E_{1} I_{1} m_{2} \mu^{2} \omega^{2}-E_{1} I_{1} m_{2} \omega^{2}-E_{2} I_{2} m_{1} \omega^{2}+E_{1} I_{1} c+E_{2} I_{2} c}{E_{1} I_{1} E_{2} I_{2}} \\
& a_{3}=\frac{m_{1} m_{2} \mu^{4} \omega^{4}-c m_{2} \mu^{4} \omega^{2}-m_{1} m_{2} \mu^{2} \omega^{4}+2 c m_{1} \mu^{2} \omega^{2}+c m_{2} \mu^{2} \omega^{2}}{E_{1} I_{1} E_{2} I_{2}} \\
& a_{4}=\frac{-m_{1} m_{2} \mu^{2} \omega^{4}+c m_{2} \mu^{2} \omega^{2}+m_{1} m_{2} \omega^{4}-c m_{1} \omega^{2}-c m_{2} \omega^{2}}{E_{1} I_{1} E_{2} I_{2}}
\end{aligned}
$$


It is required that the roots of $(A 5.3)$ be either [complex \& real] or [complex] for dynamic stiffness matrix to be formulated but seem to be elusive at this stage and would be considered in future. 